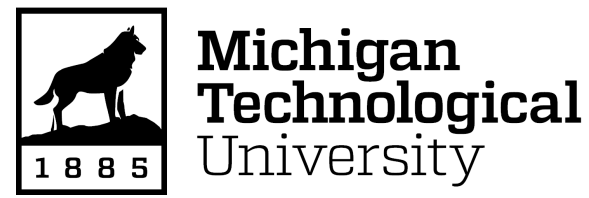

Michigan Technological University Digital Commons @ Michigan Tech

Dissertations, Master's Theses and Master's Reports

2016

\title{
Effect of Fiber Orientation on Dynamic Compressive Properties of an Ultra-High Performance Concrete
}

Andrew B. Groeneveld

Michigan Technological University, abgroene@mtu.edu

Copyright 2016 Andrew B. Groeneveld

\section{Recommended Citation}

Groeneveld, Andrew B., "Effect of Fiber Orientation on Dynamic Compressive Properties of an Ultra-High Performance Concrete", Open Access Master's Thesis, Michigan Technological University, 2016.

https://doi.org/10.37099/mtu.dc.etdr/163

Follow this and additional works at: https://digitalcommons.mtu.edu/etdr

Part of the Structural Engineering Commons 
EFFECT OF FIBER ORIENTATION ON DYNAMIC COMPRESSIVE PROPERTIES

OF AN ULTRA-HIGH PERFORMANCE CONCRETE

\author{
By
}

Andrew B. Groeneveld

\begin{abstract}
A THESIS
Submitted in partial fulfillment of the requirements for the degree of

MASTER OF SCIENCE

In Civil Engineering

MICHIGAN TECHNOLOGICAL UNIVERSITY

2016
\end{abstract}

(C)2016 Andrew B. Groeneveld 

This thesis has been approved in partial fulfillment of the requirements for the Degree of MASTER OF SCIENCE in Civil Engineering.

Department of Civil and Environmental Engineering

Thesis Advisor: $\quad$ Dr. Theresa M. Ahlborn

Committee Member: $\quad$ Dr. C. Kennan Crane

Committee Member: $\quad$ Dr. Stephen L. Kampe

Department Chair: $\quad$ Dr. David W. Hand 



\section{TABLE OF CONTENTS}

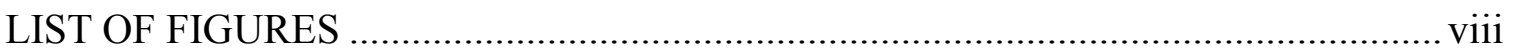

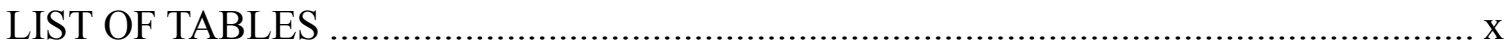

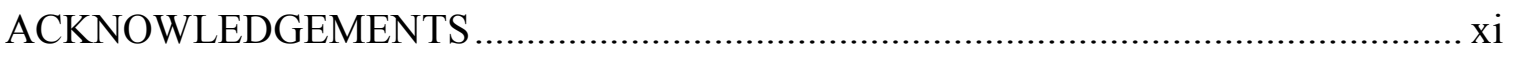

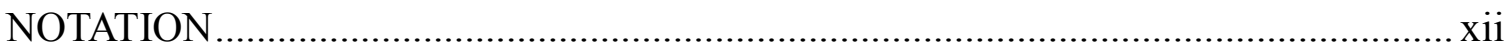

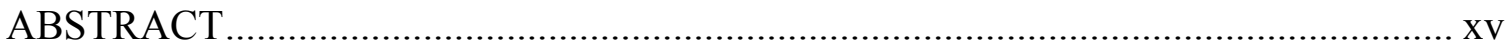

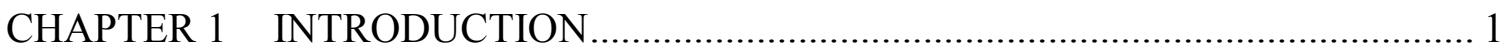

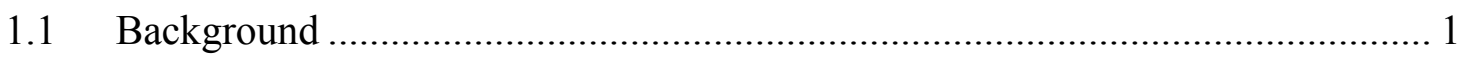

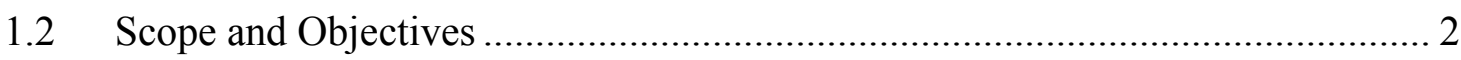

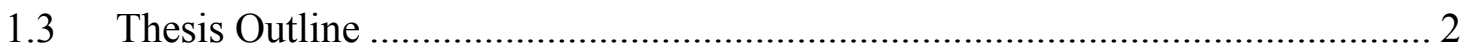

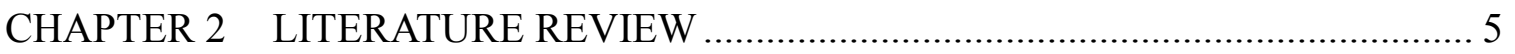

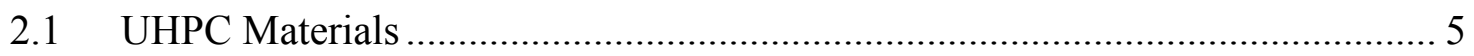

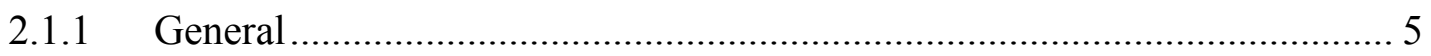

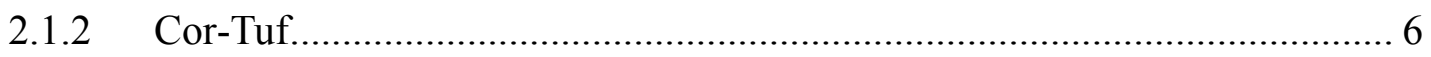

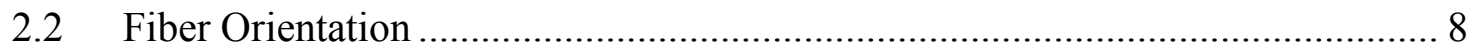

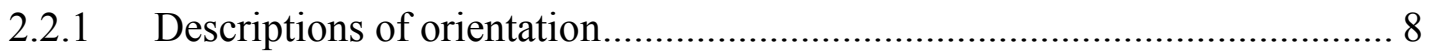

2.2.2 Methods of characterizing orientation .................................................... 10

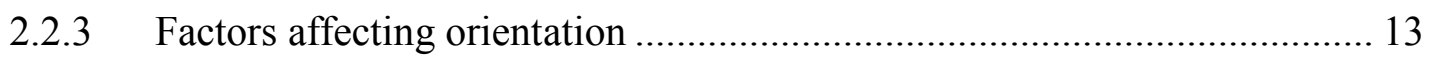

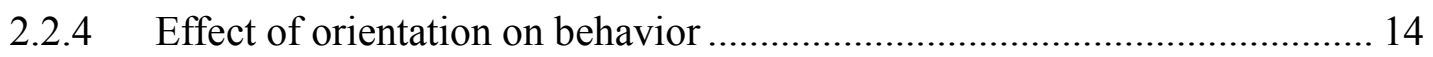

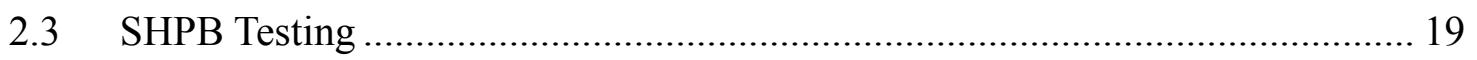

2.3.1 Historical development ..................................................................... 19

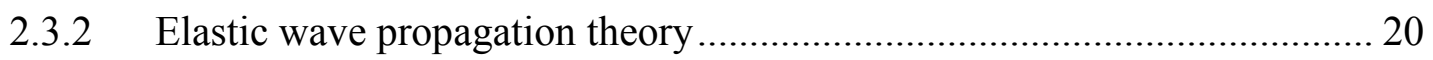

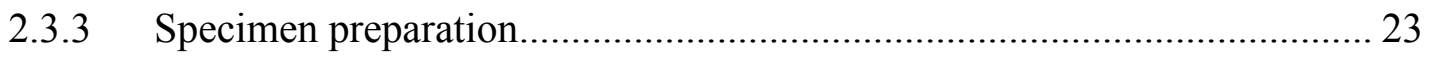

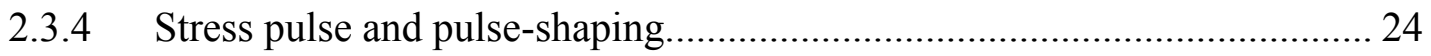

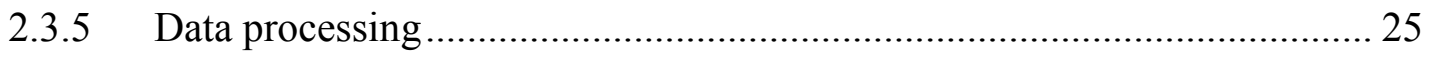

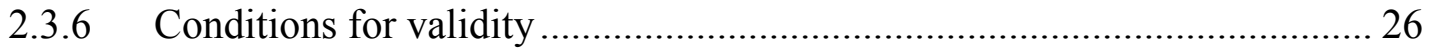

2.3.7 Stress equilibrium .......................................................................... 27

2.4 Dynamic Compressive Behavior of UHPC .................................................... 28

$2.5 \quad \mathrm{X}$-Ray Computed Tomography …………………...................................... 31

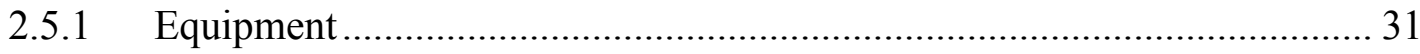

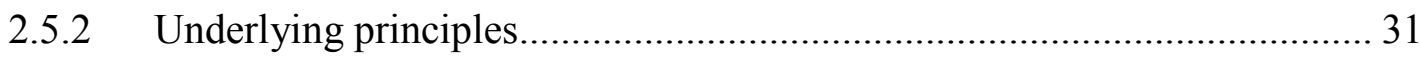

2.5.3 Application to cementitious materials …………….................................. 33

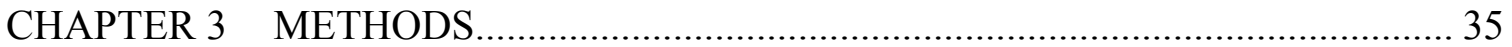




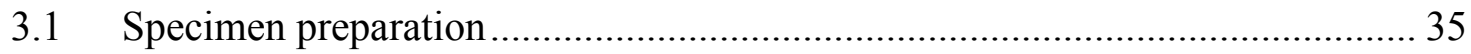

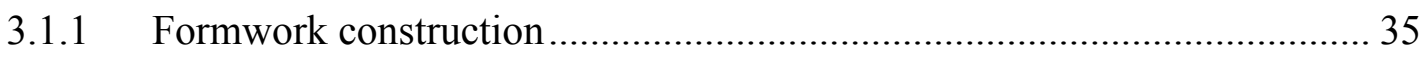

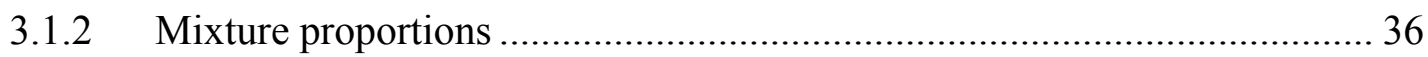

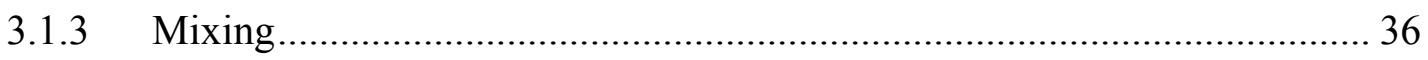

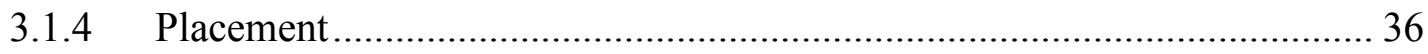

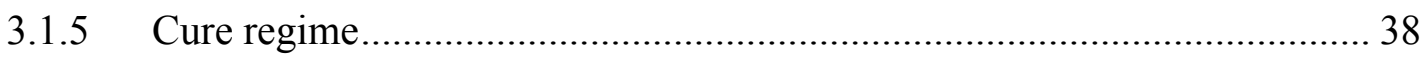

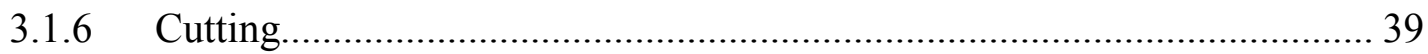

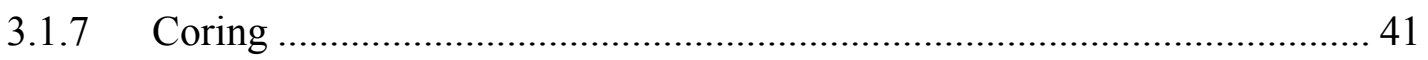

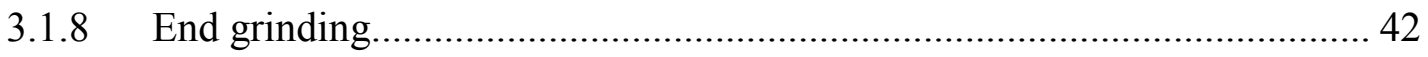

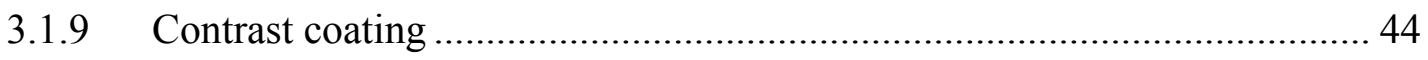

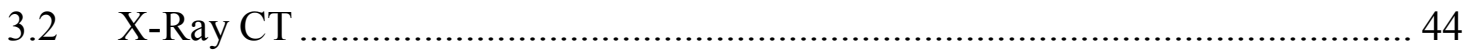

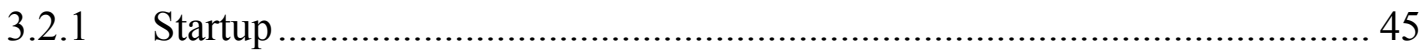

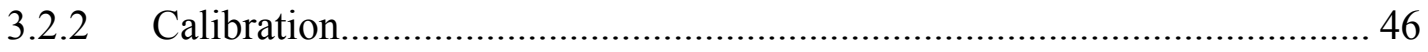

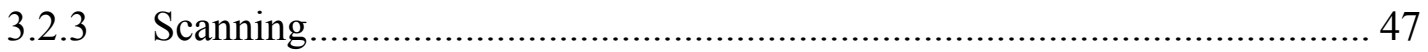

3.2.4 Calibration scan ............................................................................. 49

3.2.5 Volume reconstruction ........................................................................ 49

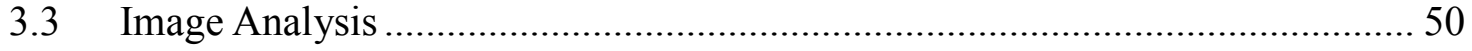

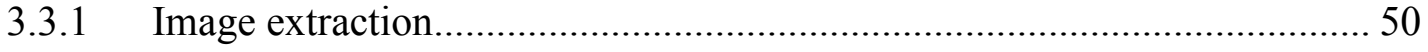

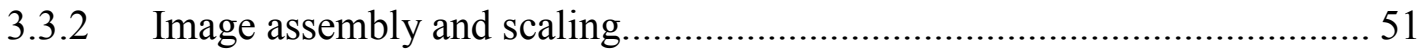

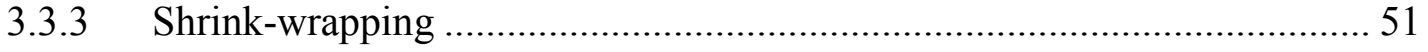

3.3.4 Image correction and void analysis...................................................... 52

3.3.5 Fiber correction and identification ......................................................... 52

3.3.6 Fiber orientation analysis I (cluster) …………........................................ 53

3.3.7 Fiber orientation analysis II (workstation)................................................. 53

3.4 Quasistatic Compression Testing ................................................................... 54

3.5 Dynamic Compression Testing ................................................................. 55

3.5.1 Bridge amplifier and meter unit calibration............................................... 57

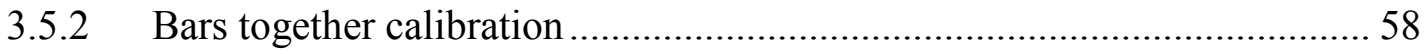

3.5.3 Bars apart calibration ......................................................................... 59

3.5.4 Specimen testing ............................................................................... 59

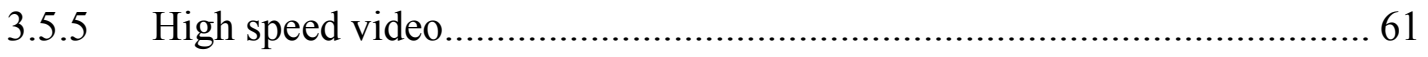

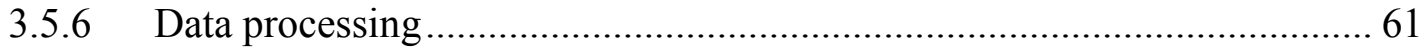

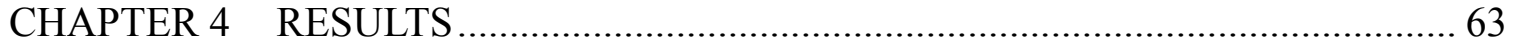




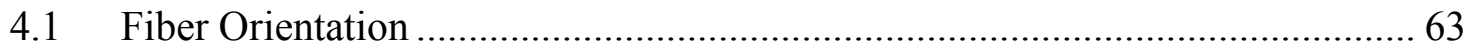

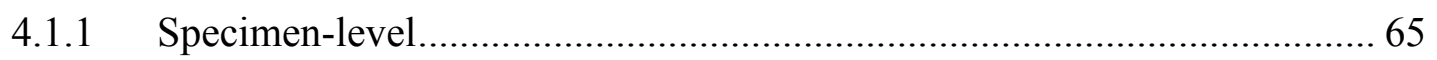

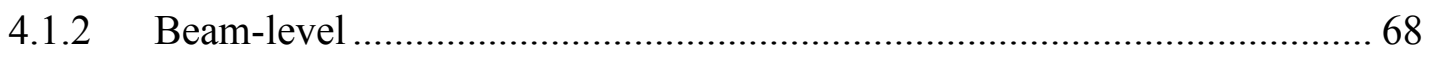

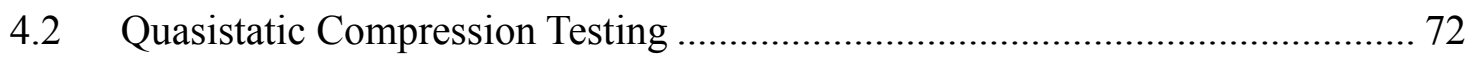

4.3 Dynamic Compression Testing ……………............................................ 76

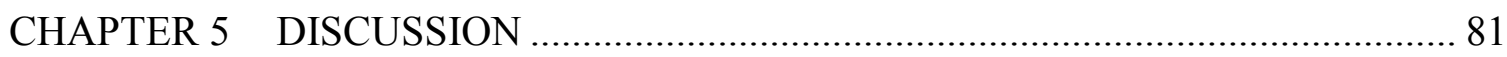

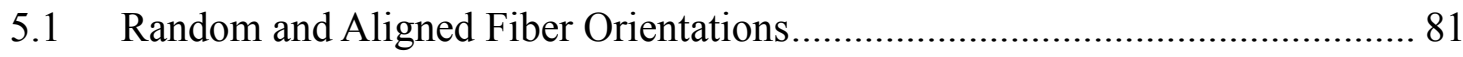

5.2 Variation of Fiber Orientation throughout Beam ………................................ 86

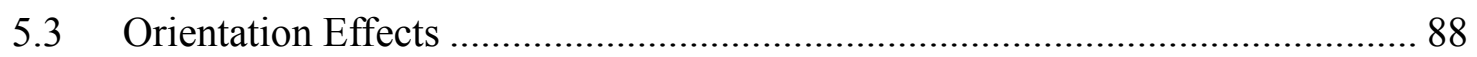

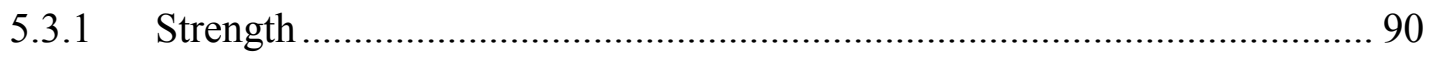

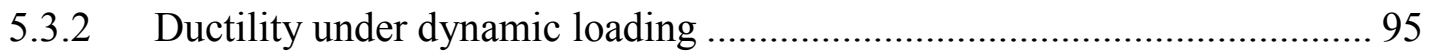

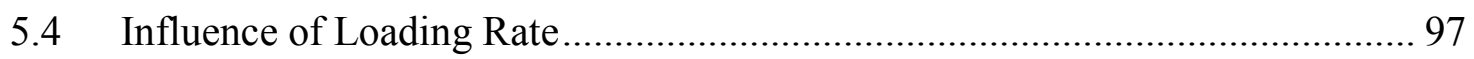

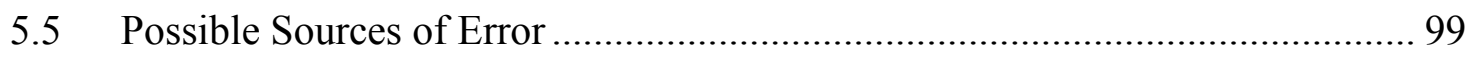

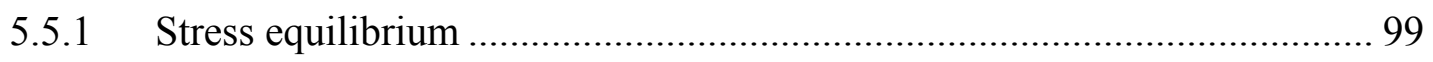

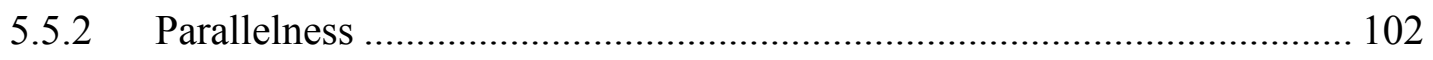

5.5.3 Radial confinement due to inertia ......................................................... 102

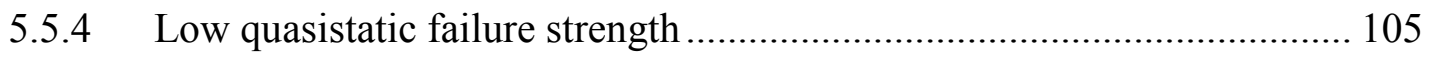

CHAPTER 6 CONCLUSIONS AND RECOMMENDATIONS ………………........ 107

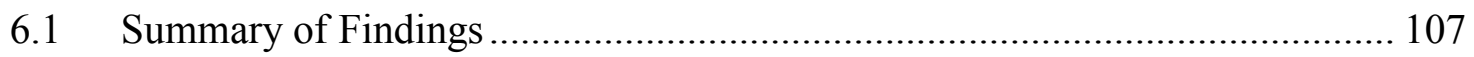

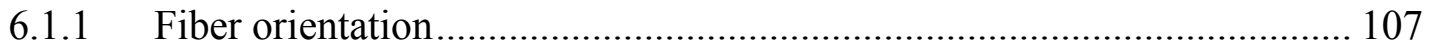

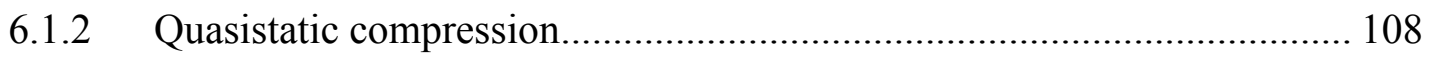

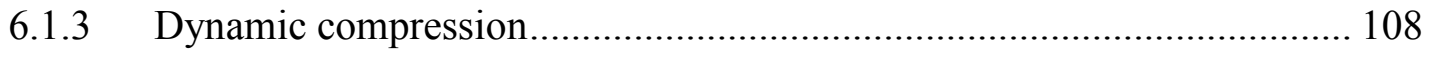

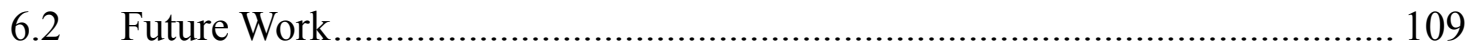

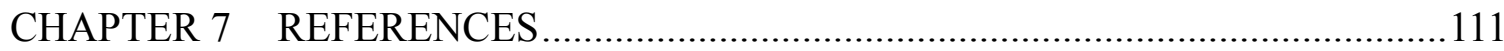

APPENDIX A End Parallelness Measurements .......................................................119

APPENDIX B X-Ray CT Scan Settings ............................................................... 121

APPENDIX C Code Scaling Pilot Study …………............................................ 124

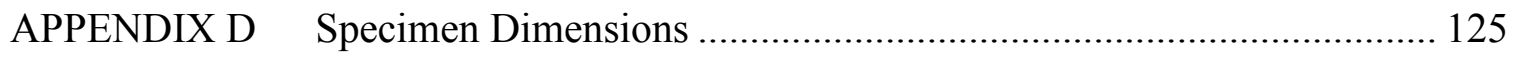

APPENDIX E Numerical Differentiation.............................................................. 127

APPENDIX F Permission for Use Of Copyrighted Material..................................... 128 


\section{LIST OF FIGURES}

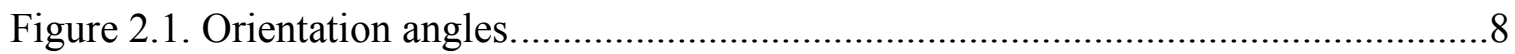

Figure 2.2. Uncertainty of fiber angle with surface normal vector............................... 11

Figure 2.3. The split-Hopkinson pressure bar............................................................20

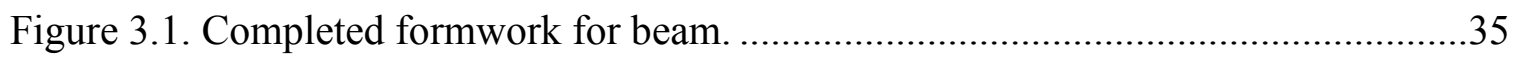

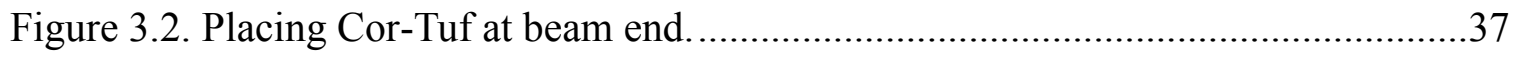

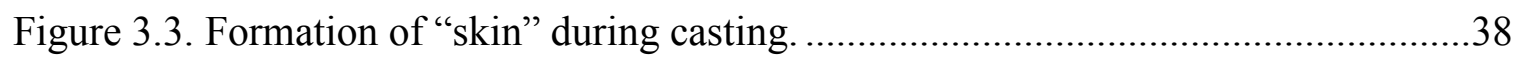

Figure 3.4. Overall schematic view of beam showing grid for specimen IDs and

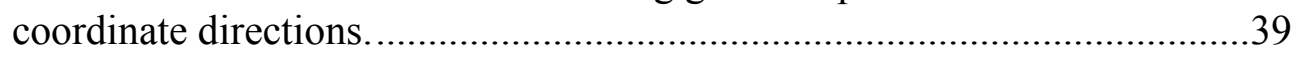

Figure 3.5. Sawing Systems Model 521C gantry saw. ...............................................40

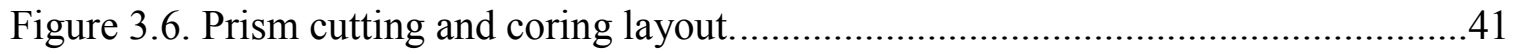

Figure 3.7. Core drills mounted to work table....................................................42

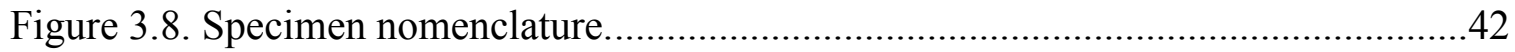

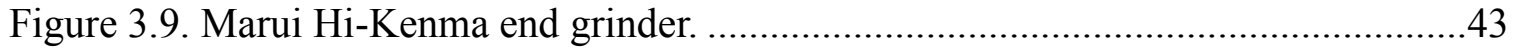

Figure 3.10. Gauges for measuring end parallelness ..............................................44

Figure 3.11. Image acquisition geometry..............................................................48

Figure 3.12. SHPB instrument looking toward output bar........................................56

Figure 3.13. Strain gage location (after Clark 2013).................................................56

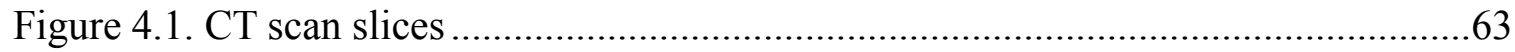

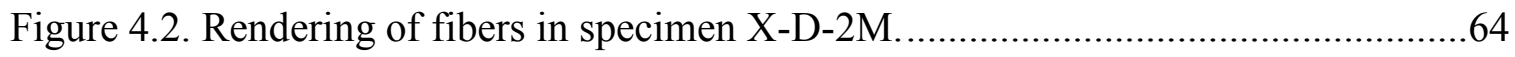

Figure 4.3. Variation of mean $x$-direction orientation angle with distance from casting end

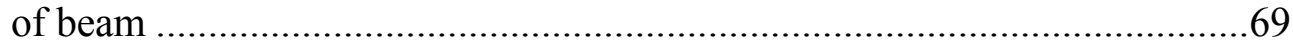

Figure 4.4. Variation of mean $y$-direction orientation angle with distance from casting end

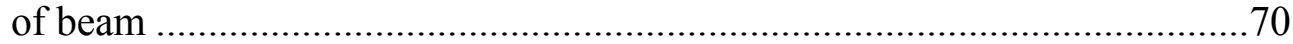

Figure 4.5. Variation of mean $z$-direction orientation angle with distance from casting end

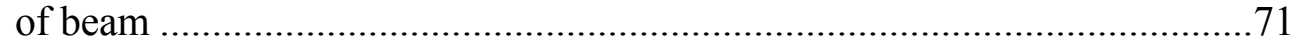

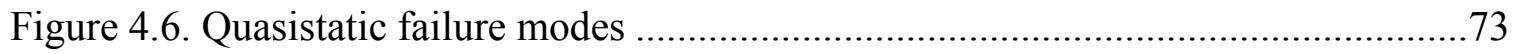

Figure 4.7. High speed video frames showing failure of specimen Y-D-4T. ..................77

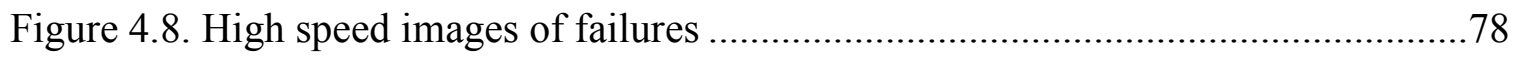

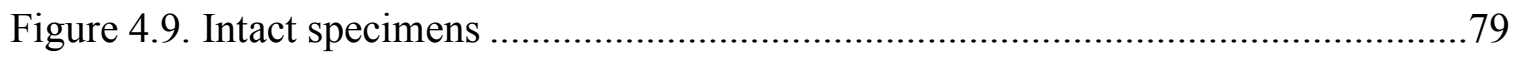

Figure 4.10. Examples of truncated type 2 failures. ................................................. 80

Figure 5.1. Theoretical probability density function for fiber orientation angle. .............81

Figure 5.2. Theoretical cumulative distribution function for fiber orientation angle. .......82

Figure 5.3. Measured orientation angle distribution for specimen X-D-2M ...................84

Figure 5.4. Cumulative distribution of orientation angle for specimen X-D-2M .............85

viii 
Figure 5.5. Illustration of parallel and perpendicular orientation number. .89

Figure 5.6. Variation of quasistatic compressive strength ........................................90

Figure 5.7. Specimens with low quasistatic strength .................................................... 91

Figure 5.8. Fiber effect on quasistatic strength for different failure modes.....................92

Figure 5.9. Two fibers at 45 degrees from the axis of loading. .....................................92

Figure 5.10. Variation of dynamic compressive strength.........................................95

Figure 5.11. Stress-strain curves for all dynamic compression tests. ............................96

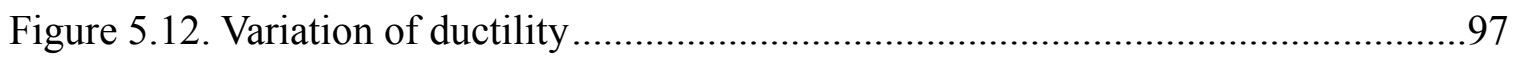

Figure 5.13. Illustration of some loading pulse forms...........................................101

Figure 5.14. Axial strain rate and strain acceleration for specimen X-D-2M.................102

Figure 5.15. Axial strain rate and strain acceleration using a seven-point Savitzky-Golay filter for specimen X-D-2M........................................................... 103

Figure C.1. Timing measurements for LSFfiberOrient function for multiple cores........124 


\section{LIST OF TABLES}

Table 2.1. Cor-Tuf material properties (Williams et al. 2009)..........................................

Table 2.2. Orientation efficiency factors for simple arrangements of fibers. ....................

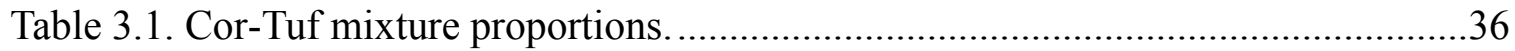

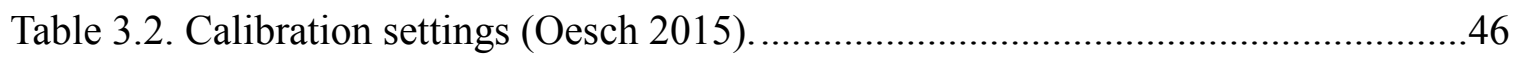

Table 3.3. Typical range of settings for $\mathrm{x}$-ray CT scanning. ............................................47

Table 3.4. Typical settings for calibration scanning.....................................................49

Table 4.1. Fiber orientation angles (degrees) calculated from CT scan data. ..................66

Table 4.2. Specimen composition by volume from CT scan data..................................67

Table 4.3. Quasistatic compressive strength of Cor-Tuf specimens ..............................72

Table 4.4. Quasistatic compressive strength of Cor-Tuf specimens bearing on steel........74

Table 4.5. Quasistatic compressive strength of Cor-Tuf control specimens .....................75

Table 4.6. Dynamic compressive strength of Cor-Tuf specimens. ....................................76

Table 5.1. Results of Games-Howell comparison for average orientation angle..............86

Table 5.2. Results of Games-Howell comparison for average $x$-direction orientation

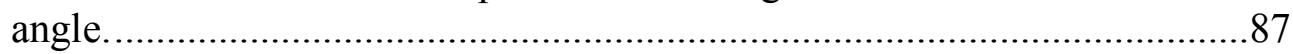

Table 5.3. Results of multiple comparisons method for equal variance. .........................88

Table 5.4. Results of Games-Howell comparison for quasistatic compressive strength. ..94

Table 5.5. DIFs using core quasistatic strengths as the reference...................................98

Table 5.6. Results of Games-Howell comparison for DIF............................................99

Table 5.7. Stress equilibrium during dynamic compression. ........................................100

Table 5.8. Calculated confining stress due to radial inertia..........................................105

Table A.1. Quasistatic compression test specimen parallelness measurements..............119

Table A.2. Additional quasistatic compression test specimen parallelness

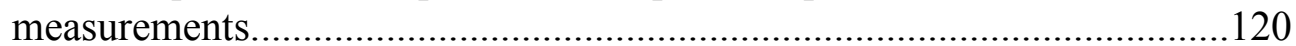

Table A.3. Dynamic compression test specimen parallelness measurements.................120

Table B.1. Settings for X-ray CT scans performed in December 2015 .......................121

Table B.2. Settings for X-ray CT scans performed in February 2016. ..........................122

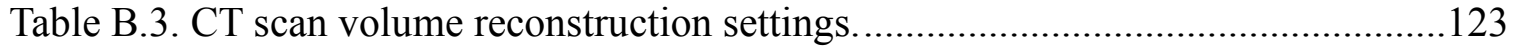

Table D.1. Quasistatic compression test specimen dimensions..................................125

Table D.2. Additional quasistatic compression test specimen dimensions.....................125

Table D.3. Dynamic compression test specimen dimensions.....................................126

Table D.4. Dynamic compression test specimen weights..........................................126 


\section{ACKNOWLEDGEMENTS}

I have had the privilege of working with outstanding people from several organizations: Michigan Technological University (Michigan Tech), the University of Florida (UF), the University of Maine (UMaine), and the U.S. Army Engineer Research and Development Center - Geotechnical and Structures Laboratory (ERDC/GSL). I would first like to thank my advisor, Dr. Tess Ahlborn (Michigan Tech), and my committee members, Drs. Kennan Crane (ERDC/GSL) and Steven Kampe (Michigan Tech), for their guidance and encouragement. Thanks are also due to Messrs. Rudy Andreatta, David Miller, and Tyler Temple (all ERDC/GSL) for their help in specimen preparation, and to Ms. Wendy Long and Messrs. Dylan Scott and Kirk Walker (all ERDC/GSL) for their assistance with casting the beam. I am grateful to Mr. Charlie Burchfield (ERDC/GSL) and Dr. Eric Landis (UMaine) for performing x-ray CT scans in December 2015 when I was unable to be at UF, and for training me in use of the equipment. Thanks also go to Mr. George Lopp and Ms. Jeremy Magruder (both UF) for their expertise in troubleshooting and repairing the x-ray CT machine. Finally, I would like to thank Messrs. Henrique "Kiko" de Melo e Silva and Rob Fritz (both Michigan Tech) for training and assistance in the Benedict Lab, Mr. David Miller (ERDC/GSL) for running high speed video and helping me set up the dynamic tests, and Mr. Richard Magee, Jr. (ERDC/GSL) for giving great advice and lending high speed video equipment.

Funding through ERDC/GSL for materials and x-ray CT scanner access, as well as financial support from the Department of Defense SMART Scholarship, are both gratefully acknowledged. 


\section{NOTATION}

The following symbols are used in this document. When a symbol has multiple definitions, the definitions are listed in order of appearance. In the text, the definition should be clear based on context.

\section{Roman Symbols}

\begin{tabular}{ll}
$A_{b}$ & Cross-sectional area of bar \\
$A_{f}$ & Cross-sectional area of fiber \\
$A_{s}$ & Cross-sectional area of specimen \\
$\boldsymbol{a}$ & Fiber orientation unit vector \\
$a_{n}$ & Fraction of fibers oriented at $\theta_{n}$ \\
$C A L S E T$ & Calibration setting number for bridge amplifier and meter (BAM) unit \\
$c_{0, b}$ & Elastic wave speed of bar \\
$c_{0, s}$ & Elastic wave speed of specimen \\
$c_{0, s t}$ & Elastic wave speed of striker \\
$D_{1}$ & Distance from X-ray source to specimen centerline \\
$D_{2}$ & Distance from specimen centerline to x-ray detector \\
$D_{s}$ & Diameter of undeformed specimen \\
$D I F$ & Dynamic increase factor \\
$d$ & Fiber diameter \\
$E$ & Elastic modulus \\
$E_{b}$ & Elastic modulus of bar \\
$E_{s}$ & Elastic modulus of specimen \\
$E_{s t}$ & Elastic modulus of striker \\
$F(\theta)$ & Cumulative distribution function \\
$f(\theta)$ & Probability density function \\
$f_{c}^{\prime}$ & 28-day unconfined compressive strength \\
$f_{c d}$ & Peak stress during dynamic compression test \\
$f_{c s}$ & Quasistatic unconfined compressive strength \\
$f_{t s}$ & Quasistatic tensile strength \\
$G$ & Shear modulus \\
$G F$ & Gage factor \\
$I / I_{0}$ & Ratio of final x-ray intensity to initial x-ray intensity \\
$I_{d}$ & Dark field intensity for x-ray detector \\
$I_{m a x}$ & Maximum intensity cutoff for scaling \\
$I_{m i n}$ & Minimum intensity cutoff for scaling \\
$K$ & Bulk modulus \\
$K_{\varepsilon}$ & Strain correction factor \\
$K_{\sigma}$ & Stress correction factor \\
$k$ & Number of wave transits \\
\hline
\end{tabular}




$\begin{array}{ll}L_{s} & \text { Length of undeformed specimen } \\ L_{S t} & \text { Length of striker } \\ \ell & \text { Fiber length } \\ \ell_{S} & \text { Length required for full anchorage of fibers } \\ M & \text { Length of the major axis of an ellipse } \\ m & \text { Length of the minor axis of an ellipse } \\ N & \text { Number of gages active during calibration } \\ N & \text { Total number of fibers } \\ N_{A} & \text { Number of fibers per unit area } \\ P_{1} & \text { Force at input bar-specimen interface } \\ P_{2} & \text { Force at specimen-output bar interface } \\ p & \text { Probability of randomly observing given data if the null hypothesis is true } \\ R^{2} & \text { Coefficient of determination } \\ R_{g} & \text { Gage resistance } \\ r & \text { Distance measured from center of specimen } \\ T & \text { Theoretical duration of incident pulse } \\ t & \text { Time } \\ u & \text { Displacement along } x \text {-axis } \\ V_{f} & \text { Fiber volume fraction } \\ V_{s t} & \text { Striker velocity } \\ V a r(\cdot) & \text { Variance operator } \\ x & \text { Cartesian coordinate } \\ x & \text { Conventional notation for specimen thickness in Beer-Lambert Law } \\ y & \text { Cartesian coordinate } \\ Z & \text { Cartesian coordinate }\end{array}$

\section{Greek Symbols}

$\alpha \quad$ Probability of making a type I error (also called the level of significance)

$\alpha(t) \quad$ Function defined in equation (2.16), $\alpha(t)=\mu /\left(L_{s} / D_{s}\right) \cdot\left[1+\varepsilon_{s}(t)\right]^{-3 / 2}$

$\beta \quad$ Parameter for wave dispersion correction

$\beta \quad$ Relative acoustic impedance

$\gamma \quad$ Dry unit weight

$\Delta \sigma \quad$ Difference in engineering stress between the specimen ends

$\varepsilon_{c r} \quad$ Value of $\varepsilon_{s}$ at peak stress (also called critical strain)

$\varepsilon_{I} \quad$ Engineering strain in bar due to incident pulse

$\varepsilon_{R} \quad$ Engineering strain in bar due to reflectd pulse

$\varepsilon_{S} \quad$ Average engineering strain in specimen

$\varepsilon_{T} \quad$ Engineering strain in bar due to transmitted pulse

$\eta \quad$ Orientation number (also called efficiency factor by some authors)

$\eta^{\prime} \quad$ Total efficiency factor, $\eta^{\prime}=\eta \eta_{\ell}$

$\eta_{\ell} \quad$ Efficiency modification for fiber length

$\eta_{\|} \quad$ Orientation number for fibers parallel to the axis of loading

$\eta_{\perp} \quad$ Orientation number for fibers perpendicular to the axis of loading 


\begin{tabular}{ll}
$\theta$ & Orientation angle \\
$\theta_{x}$ & Orientation angle measured relative to $x$-axis \\
$\theta_{y}$ & Orientation angle measured relative to y-axis \\
$\theta_{z}$ & Orientation angle measured relative to $z$-axis \\
$\mu$ & Coefficient of friction \\
$\mu \varepsilon_{C A L}$ & Simulated strain in calibration \\
$\mu / \rho$ & Mass attenuation coefficient for x-ray radiation \\
$\nu$ & Poisson's ratio \\
$v_{s}$ & Poisson's ratio of specimen \\
$\rho$ & Mass density \\
$\rho_{b}$ & Mass density of bar \\
$\rho_{s}$ & Mass density of specimen \\
$\rho_{S t}$ & Mass density of striker \\
$\sigma_{1}$ & Engineering stress at input bar-specimen interface \\
$\sigma_{2}$ & Engineering stress at specimen-output bar interface \\
$\sigma_{a}$ & Apparent (i.e., measured) engineering stress \\
$\dot{\sigma}_{A S T M}$ & Rate of stress application specified by ASTM standard, $35 \mathrm{psi} / \mathrm{s}$ \\
$\sigma_{f}$ & Uniaxial tensile strength of fiber \\
$\sigma_{I}$ & Engineering stress in bar due to incident pulse \\
$\sigma_{m}$ & Average of engineering stresses at ends of specimen \\
$\sigma_{o}$ & Actual engineering stress after correction \\
$\sigma_{r}$ & Radial engineering stress due to radial inertia \\
$\sigma_{s}$ & Engineering stress in specimen \\
$\dot{\sigma}_{U H P C}$ & Rate of stress application for UHPC testing, $150 \mathrm{psi} / \mathrm{s}$ \\
$\tau$ & Total attenuation \\
$\tau$ & Specimen transit time \\
$\tau_{o}$ & Maximum bond strength between fiber and matrix \\
$\phi$ & Azimuth angle (also called the in-plane angle) \\
$\chi(\eta)$ & Ratio of tensile strength at orientation $\eta$ to the tensile strength with fibers \\
& perfectly aligned \\
& \\
\hline
\end{tabular}

\section{Subscripts and Superscripts}

\begin{tabular}{|c|c|}
\hline $\max$ & Maximum value during a test \\
\hline measured & Measured value from a calibration run \\
\hline theoretical & Theoretical value corresponding to the measured calibration value \\
\hline (single overdot) & Indicates differentiation once with respect to time \\
\hline "(double overdot) & Indicates differentiation twice with respect to time \\
\hline - (overbar) & Indicates arithmetic mean \\
\hline
\end{tabular}




\section{ABSTRACT}

Casting structural elements with ultra-high performance concrete (UHPC) tends to create preferential fiber alignment, which affects the strength and must be accounted for in design. Fiber orientation effects in tension have been studied extensively, but less work has been performed for compression. This work characterizes the fiber orientation occurring in a typical UHPC beam and how that orientation affects compressive behavior at high strain rates. Specimens (36 total) were cored from the beam and their fiber orientations were non-destructively evaluated using x-ray computed tomography (CT). Fibers showed flow-induced alignment along the length of the beam, with orientation angles in the $x, y$, and $z$-directions differing significantly. The perpendicular orientation number was used to describe orientation, as fibers perpendicular to the load were most effective in crack bridging. Cored specimens tested quasistatically achieved compressive strengths of 14.3-23.6 ksi, which appeared to increase with perpendicular orientation number. However, limited data makes this correlation uncertain. Quasistatic strengths were lower than expected due to the use of neoprene pads. Quasistatic strengths of cores tested without pads averaged $26.6 \mathrm{ksi}$. Dynamic tests at strain rates of 130-200 1/s were performed with a split-Hopkinson pressure bar (SHPB). Dynamic compressive strength ranged from $38.1 \mathrm{ksi}$ to $58.5 \mathrm{ksi}$ and was independent of orientation number, although results suggested that the distribution and orientation of fibers influenced crack formation. The strain at peak stress, a measure of ductility, ranged from 0.0105 to 0.0131 in dynamic tests. Strain at peak stress increased with perpendicular orientation number, but the correlation was weak. Sources of error, including stress non-equilibrium and radial confinement due to inertia, were assessed. 



\section{CHAPTER 1 INTRODUCTION}

\subsection{Background}

Ultra-high performance concrete (UHPC) was developed to overcome some of the limitations of normal strength concrete (NSC), which include long-term durability and tensile strength. Steel fibers are used in UHPC to bridge cracks, thereby increasing tensile strength and providing tensile capacity once cracks open. When structural elements are cast with UHPC, the flow of material tends to cause fiber alignment (Martinie and Roussel 2011). Previous works at quasistatic rates of loading have shown that the tensile strength (Kang and Kim 2011) and compressive strength (Mansur et al. 1999; Empelmann et al. 2008; Leonhardt et al. 2012) of UHPC are affected by the fiber orientation. In general, fibers are most effective in strengthening a matrix when the fibers are parallel to a tensile stress. When a compressive stress is applied, tensile cracks will be caused instead by Poisson expansion, as well as wing-crack growth (Victor C. Li 1992). Thus, fibers that are perpendicular to the compressive stress are most effective. Typical design practice is to assume that the material is isotropic, but this assumption may be unconservative. Recognizing this, the French Association of Civil Engineers (AFGC 2013) recommends the use of a K-factor, which is an empirically-determined adjustment for the effect of fiber orientation on the material's tensile behavior. To the author's best knowledge, no such adjustment has been proposed for compressive behavior.

UHPC is also a rate-sensitive material, exhibiting higher failure strengths at higher rates of loading (Rong et al. 2010), which may occur during earthquakes or impacts. It is possible that the effects of fiber orientation may be different at higher rates of loading. Dynamic strength is very sensitive to defects in the material, and fibers can act as voids to initiate failure. Also, at high rates of loading, specimens are in a state of uniaxial strain, rather than uniaxial stress (Field et al. 2004), which may alter the load transfer between the matrix and fibers. 
The fiber orientation in structural elements is affected by the methods of placement and compaction, as well as the shape of the form. Therefore, the fiber orientation is not likely to be isotropic, and may in fact vary throughout the beam. For designers to safely use UHPC, there is a need for information on the expected compressive strength taking fiber orientation into account. Such information is especially valuable when designing for extreme loadings, such as seismic or impact events. Further, when combined with an approach for predicting fiber alignment-such as that proposed by Laranjeira et al. (2012) - areas with a lower strength due to fiber effects can be identified in structural elements. The reduced strength can then be accounted for in the design. The reliability of the structure is increased by using a design capacity that more accurately reflects the capacity of the actual structure.

\subsection{Scope and Objectives}

The overarching goal of this project was to determine the effects of fiber orientation on the dynamic strength and ductility of Cor-Tuf, a UHPC developed by the U.S. Army Corps of Engineers. This goal was broken into three primary objectives: first, to document the fiber orientation occurring in a Cor-Tuf beam cast according to typical practice; second, to determine the effect of fiber orientation on the dynamic compressive behavior of Cor-Tuf; and, third, to determine if the effects of fiber orientation are different between quasistatic and dynamic loading rates.

\subsection{Thesis Outline}

The remaining chapters of this thesis are organized as follows.

Chapter 2 presents a literature review. A general description of UHPC is given, with emphasis on current research related to Cor-Tuf. Research on methods of characterizing fiber orientation is summarized. The theory and application of the splitHopkinson pressure bar (SHPB), a method of high strain rate testing, are explored. Lastly, x-ray computed tomography (CT) and its use for cementitious materials are reviewed. 
Chapter 3 describes the experimental methods employed. Specimen preparation is covered in some detail, beginning with casting the beam. X-ray CT was used to nondestructively image the specimens' internal structure, and image processing techniques were used to determine the orientation of fibers within the specimens. Quasistatic compression testing was performed with a hydraulic compression testing machine, and dynamic compression testing was performed with an SHPB.

Chapter 4 presents results for fiber orientation angles, quasistatic compressive strength, and dynamic compressive strength and ductility. Failure modes at the two loading rates are examined. Also, high speed video is used to illustrate the failure of specimens.

Chapter 5 discusses the results in greater depth. Trends in fiber orientation in the beam are examined, and the effect of orientation on compressive properties under quasistatic and dynamic loading is analyzed. The influence of loading rate on the orientation effects is also considered. Possible sources of error are addressed, including non-parallel specimen ends, stress non-equilibrium, and radial confinement due to inertia.

Chapter 6 summarizes the main findings from this research. Questions that arose during the study, but were outside its scope, are identified as possible topics for future work. 



\section{CHAPTER 2 LITERATURE REVIEW}

\subsection{UHPC Materials}

\subsubsection{General}

UHPC is designed to be used for applications where the use of NSC would be impractical or impossible. Development of UHPCs began in Europe. In France, researchers at Bouygues $^{1}$ formulated reactive powder concrete (RPC). RPC was designed to have a homogeneous, high-density microstructure to increase compressive strength, and incorporated steel fibers to increase tensile strength and ductility (Richard and Cheyrezy 1995). The term "reactive powder" refers to the use of silica fume, which produces secondary hydrates through a pozzolanic reaction, and finely crushed quartz, which reacts with amorphous hydrates to form tobermorite (Richard and Cheyrezy 1995).

Typical distinguishing features of a UHPC are careful control of particle size, low water-to-cement ratio (w/c), and the inclusion of fibers to improve tensile properties. Because UHPC technology is still developing, there is not a single accepted definition. Two, somewhat overlapping, definitions are presented here for illustration. The Federal Highway Administration (FHWA) defines UHPC in terms of its composition, mechanical properties, and a durability requirement:

UHPC is a cementitious composite material composed of an optimized gradation of granular constituents, a water-to-cementitious materials ratio less than 0.25 , and a high percentage of discontinuous internal fiber reinforcement. The mechanical properties of UHPC include compressive strength greater than $21.7 \mathrm{ksi}(150 \mathrm{MPa})$ and sustained postcracking tensile strength greater than $0.72 \mathrm{ksi}(5 \mathrm{MPa})$. UHPC has a discontinuous pore structure that reduces liquid ingress, significantly enhancing durability as compared to conventional and high-performance concretes. (Graybeal 2011)

\footnotetext{
${ }^{1}$ Pronounced bweeg.
} 
American Concrete Institute (ACI) Committee 239 has developed a working definition (pending approval) for UHPC: "concrete that has a minimum specified compressive strength of $150 \mathrm{MPa}(22,000 \mathrm{psi})$ with specified durability, tensile ductility and toughness requirements; fibers are usually required to achieve specified requirements" (ACI 239 2015). Note that the use of fibers (typically steel) does not make a certain mixture a UHPC; work on fiber-reinforced concrete (FRC) has been going on since at least the early 1960s (Zollo 1997). Swamy (1975) reviewed the use of fiber reinforcement for concrete and noted that fibers act as defects in compression and do not appreciably increase compressive strength; however, fibers do have significant benefits in tension, and can provide a quasi-ductile failure by bridging cracks.

\subsubsection{Cor-Tuf}

Cor-Tuf was designed by the U.S. Army Corps of Engineers to provide a standard, wellcharacterized UHPC that can be consistently produced (Williams et al. 2009). Cor-Tuf can be characterized as RPC and has no coarse aggregate: the largest particles are foundry-grade Ottawa silica sand with a diameter of about $600 \mu \mathrm{m}(0.024 \mathrm{in}$.). The mix has a w/c of 0.21 , and uses a superplasticizer to increase its workability. Steel fibers are added to improve ductility. The chosen fibers are Bekaert Dramix ZP 305 steel fibers, which are $30 \mathrm{~mm}$ (1.2 in.) in length and $0.55 \mathrm{~mm}(0.022 \mathrm{in}$.) in diameter, and have hooked ends for better pull-out resistance. The manufacturer specifies a tensile strength of approximately $160 \mathrm{ksi}$ and an elastic modulus of 30,500 ksi. Fiber content is typically 3.6 vol\%. Full details on mixture proportions can be found in Section 3.1.

Extensive testing has been carried out to determine the mechanical properties of Cor-Tuf. Quasistatic testing on Cor-Tuf with and without fiber reinforcement has been performed for confined and unconfined compressive properties (Williams et al. 2009) as well as flexural and splitting tensile properties (Roth et al. 2010). Unconfined compressive strengths as high as $35 \mathrm{ksi}$ have been achieved in laboratory testing

(Williams et al. 2009). Selected mechanical and physical properties for Cor-Tuf (Williams et al. 2009) are given in Table 2.1 below. Curiously, the quasistatic tensile 
strengths reported by Williams et al. (2009) do not show improvement with the addition of steel fibers. This unusual trend in tensile strength is not discussed in the report.

Table 2.1. Cor-Tuf material properties (Williams et al. 2009).

\begin{tabular}{lcc}
\hline \hline \multicolumn{1}{c}{ Property } & $\begin{array}{c}\text { Cor-Tuf } \\
\text { without Fibers }\end{array}$ & $\begin{array}{c}\text { Cor-Tuf } \\
\text { with Fibers }\end{array}$ \\
\hline Bulk modulus, $K(\mathrm{ksi})$ & 3290 & 3650 \\
Shear modulus, $G(\mathrm{ksi})$ & 2220 & 2420 \\
Elastic modulus, $E(\mathrm{ksi})$ & 5440 & 5930 \\
Poisson's ratio, $v(-)$ & 0.22 & 0.23 \\
Unconfined quasistatic compressive & 30.5 & 34.4 \\
strength, $f_{c s}(\mathrm{ksi})$ & & \\
Quasistatic tensile strength, $f_{t s}(\mathrm{ksi})$ & 1.28 & 0.83 \\
Dry unit weight, $\gamma$ (pcf) & 141 & 155 \\
\hline \hline
\end{tabular}

Impact and penetration testing has been carried out on Cor-Tuf to determine damage to the phases (Moser et al. 2013) and the effect of different types of fiber reinforcement (Scott et al. 2015). Dynamic compression testing of Cor-Tuf was performed by VanSlembrouck (2015), who found that the dynamic increase factor (DIF) for Cor-Tuf ranged from about 1.85 to 2.09 . The DIF is the ratio of dynamic failure strength to quasistatic failure strength, and is discussed further in Section 2.4. Mondal (2012) performed dynamical triaxial compression (TXC) testing, using an SHPB modified to provide hydraulic radial confinement to specimens. Mondal's results show that the fracture strength increases with confinement for dynamic tests, as would be expected. Results also showed that the dynamic TXC tests gave higher fracture strength than the quasistatic TXC tests carried out by the U.S. Army Corps of Engineers (Williams et al. 2009); however, Mondal attributes this to size effects rather than rate effects. Indeed, the $19.05-\mathrm{mm}$ diameter by $12.5-\mathrm{mm}$ long $(3 / 4$-in. diameter by $1 / 2$-in. long) cylinders used by Mondal have less than $1 \%$ of the volume of the $75-\mathrm{mm}$ diameter by 150-mm long (3-in. diameter by 6-in. long) cylinders used by the Corps of Engineers. 


\subsection{Fiber Orientation}

\subsubsection{Descriptions of orientation}

This section presents terminology and various mathematical representations of orientation to provide a foundation on which to build. In general, orientation will be used to mean the direction of a fiber (or fibers) with respect to some coordinate system. Preferential alignment, or simply alignment, will refer to an orientation in which all or most of the fibers have the same direction.

The orientation of a fiber can be described by the angles it makes with each of the axes, shown as $\theta_{x}, \theta_{y}$, and $\theta_{z}$, respectively, in Figure 2.1. The azimuth or in-plane angle $\phi$ is measured from the $x$-axis to the fiber's horizontal projection in the $x y$-plane. A unit vector $\boldsymbol{a}$ may also be used to describe the fiber's orientation.

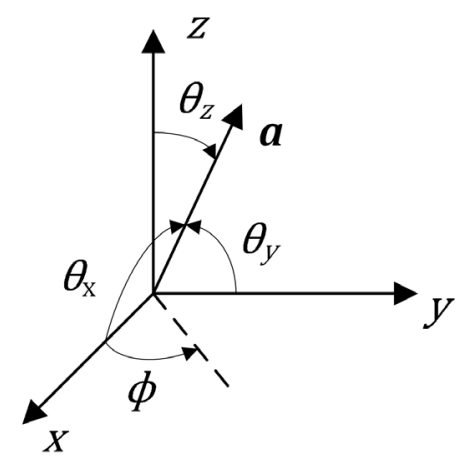

Figure 2.1. Orientation angles.

The angles and unit vector components are related as shown below:

$$
\begin{aligned}
& a_{x}=\cos \theta_{x}, \\
& a_{y}=\cos \theta_{y}, \\
& a_{z}=\cos \theta_{z}, \\
& \frac{a_{y}}{a_{x}}=\tan \phi .
\end{aligned}
$$


A single specimen may contain hundreds or thousands of fibers. Using the angle or vector for each fiber would provide a lot of detail, but would be impractical. A statistical approach might involve fitting a distribution to the observed orientations in a specimen. The mean and mode can be used to determine the overall alignment tendency, and the standard deviation can be used to determine the degree of alignment or nonalignment (Oesch 2015). The orientation number, typically $\eta$, for a given section is the ratio of the total fiber length projected along the plane's normal, to the total fiber length. This can also be thought of as an average directional cosine. Finally, even-order tensors may be used (Advani and Tucker III 1987; Lee et al. 2002). The use of tensors facilitates determining the elastic stiffness tensor, and the orientation tensor can be predicted using the rheological equation of change (Advani and Tucker III 1987).

The orientation factor $\eta$ is sometimes also called the orientation efficiency factor or efficiency factor. However, this causes some confusion, as orientation is purely geometric but efficiency depends on material properties and the type of loading. Based on geometric probability, so-called orientation efficiency factors have been derived for various simple fiber arrangements by a number of authors (Krenchel 1964; Stroeven 1978; Kameswara Rao 1979), shown in Table 2.2. Note that, owing to different definitions of efficiency, the factors vary widely. Efficiency, and the effect of orientation on mechanical behavior, will be reviewed in Section 2.2.4.

Table 2.2. Orientation efficiency factors for simple arrangements of fibers.

\begin{tabular}{ccl}
\hline \hline Arrangement & Orientation factor, $\boldsymbol{\eta}$ & \multicolumn{1}{c}{ Source } \\
\hline 2-D random & $\frac{2}{\pi} \approx 0.637$ & Kameswara Rao (1979) \\
& 0.375 & Krenchel (1964) \\
\hline \multirow{3}{*}{ 3-D random } & $\frac{4}{\pi^{2}} \approx 0.405$ & Kameswara Rao (1979) \\
& 0.500 & Stroeven (1978) \\
& 0.200 & Krenchel (1964) \\
\hline \hline
\end{tabular}




\subsubsection{Methods of characterizing orientation}

Many experimental methods are available for characterizing the orientation of fibers in cementitious composites. This section will concentrate on steel fibers, which are most commonly used in UHPCs.

Quantitative stereology, which uses geometric probability theory to determine average properties of two- and three-dimensional structures in an object from a crosssection (Stroeven 1978; 1979; 2009), is well-established and requires comparatively simple equipment. The process is destructive, as a specimen must be cut and the section polished before observation. Modern implementations use digital image analysis, either to count the number of fiber cross-sections or to determine the dimensions of the crosssections. The first approach, which gives a count of fibers per unit area, is the traditional stereological method (Krenchel 1975):

$$
\eta=\frac{N_{A} A_{f}}{V_{f}}
$$

where: $\quad N_{A}=$ number of fibers per unit area $\left(1 / \mathrm{in.}^{2}\right)$;

$A_{f}=$ cross-sectional area of fiber $\left(\right.$ in. $\left.^{2}\right)$;

$V_{f}=$ fiber volume fraction (unitless).

Gettu et al. (2005) used the fiber counting method to study the effect of casting and consolidation methods on fiber orientation and spatial distribution.

Alternatively, the second approach works by considering that a cylindrical fiber cut at an angle will have an elliptical cross-section. The angle of the fiber with respect to the section's normal is (Lee et al. 2002):

$$
\theta= \pm \arccos \frac{m}{M}
$$

where: $\quad m=$ length of the minor axis (in.);

$M=$ length of the major axis (in.).

As indicated by equation (2.3), this approach only allows determination of the magnitude of the angle. Consider two fibers making the same angle with the vertical 
axis, but with one rotated 180 degrees around that axis. These fibers would then make an "X" shape, as shown in Figure 2.2. Any horizontal section of the two fibers would look the same. Depending on the application, this uncertainty may not be an issue; however, if the in-plane angle $\phi$ is also significant, more detail is needed.

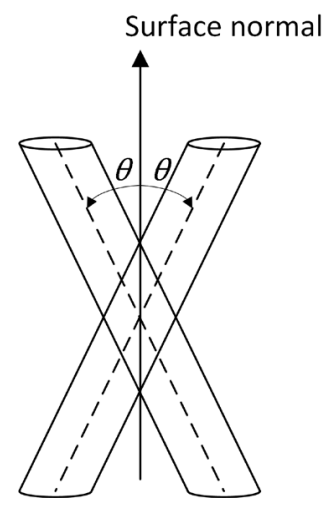

Figure 2.2. Uncertainty of fiber angle with surface normal vector.

Lee et al. (2002) overcame this uncertainty by performing image analysis at two sections, spaced about $10 \mu \mathrm{m}(0.0004$ in.) apart. This allowed the two cases mentioned before to be distinguished, because a fiber cross-section will appear at a slight offset in the second cross-section. In fact, Lee et al. (2002) were able to determine the full threedimensional orientation tensor $a_{i j}$. However, the practicality of the method is limited by the close spacing required for the cut, and by the high resolution needed to determine the dimensions of the ellipses (at least 65 pixels across the fiber diameter).

Eik et al. (2013) used a different approach to the same problem of uncertainty. Optical characterization was supplemented with a robot that used DC-conductivity probes to systematically find the end points of fibers in a $2-\mathrm{cm}(0.8$-in.) thick slice of concrete. The orientation is fully defined by knowing the coordinates of the fiber on each face. The robot was relatively inexpensive ( $€ 200$ or about $\$ 250$ ) but took roughly 36 hours to find 201 fibers making connections between the slice faces. Also, the method will miss fibers that are oriented at too steep an angle to pass through the entire slice. 
Several non-destructive approaches based on electrical conductivity have been developed. Woo et al. (2005) used the intrinsic conductivity method, which predicts the conductivity of a solid based on the volume fraction and aspect ratio of fibers. Conductivity was measured in the $x$-, $y$-, and $z$-directions to assess overall fiber orientation. Karhunen et al. (2010) applied an AC current to concrete cylinders and measured the output voltages at 16 electrodes around the perimeter. Using the distribution of resistance inside the cylinder, inclusions such as a rubber block, plastic sheet, and steel bars (in both vertical and horizontal orientations) were detected. However, determining the resistance distribution is an ill-posed inverse problem and is computationally intensive. Barnett et al. (2010) used both AC resistance tomography and $\mathrm{x}$-ray computed tomography to analyze the orientation of fibers in round slabs.

Although electrical methods are nondestructive and provide an overall value of orientation, these methods cannot give details about the individual fibers. Another technique, $\mathrm{x}$-ray computed tomography (CT), is capable of imaging the full 3-D internal structure of a specimen. X-ray CT distinguishes between phases based on X-ray absorption, which is a function of density and atomic number (for details, see Section 2.5). Schnell et al. (2008) used $\mathrm{x}$-ray CT to image concrete specimens up to $50 \times 50 \times 50$ $\mathrm{mm}(2 \times 2 \times 2 \mathrm{in}$. $)$ at a resolution of about $76 \mu \mathrm{m}(0.0030 \mathrm{in}$.). In $\mathrm{x}$-ray CT, the term voxel (for volume element) is used as the 3-D analogue of pixel (picture element). Thus, this resolution gives cubic voxels measuring $76 \mu \mathrm{m}(0.0030$ in.) on each side. Using the image analysis software MAVI developed at Fraunhofer ITWM, Schnell et al. (2008) separated the fibers from the matrix using a threshold absorption value, and then calculated fiber orientation using a discretized version of the Crofton formula from integral geometry.

Krause et al. (2010) used x-ray CT to determine the fiber orientation in a cementitious composite. After obtaining the data from the x-ray CT scan, the structure tensor was applied to the image to obtain the average gradient at a point. Because the gradient gives the direction in which the image information is changing, this allows the determination of the fiber orientation. 
Kanakubo et al. (2016) developed a method to determine the fiber orientation using a surrogate system. This work focused on FRC with polyvinyl alcohol (PVA) fibers. To observe the effect of flow on fiber orientation during casting, a transparent solution of water and waterglass (sodium silicate, a clear, viscous liquid) was substituted for the cementitious mortar. This solution was proportioned to have the same flow time as the mortar in a funnel flowability test, but the density was $86 \%$ of the mortar density. A low volume fraction of black nylon fibers was added, as the white PVA fibers had poor visibility. The surrogate FRC was cast into clear acrylic molds, and image analysis on high-resolution digital images was used to determine the orientation of fibers with the beams' longitudinal axes. This method does not require cutting or polishing, and can provide information throughout the beam. However, results were not compared with the orientation in actual FRC specimens, so the suitability of the waterglass solution for simulating mortar is not known.

\subsubsection{Factors affecting orientation}

Edgington and Hannant (1972) showed that vibration has an effect on fiber orientation. As might be expected, when vibration was applied vertically to specimens, the fibers tended to align in a horizontal plane.

The placement method also has a strong effect on fiber orientation. Barnett et al. (2010) investigated fiber orientation in round slabs, which were cast from the center, edge, and randomly. Strangely, fibers were observed to align perpendicular to the flow of fresh concrete. That is, for panels cast from the center, the concrete flowed outward radially and the fibers tended to be aligned in the hoop direction. This is in contrast to Kim et al. (2008), who cast beam and slab specimens and observed fibers aligned parallel to the direction of flow. Also, for a fiber whose axis is at some angle from the direction of flow, the drag force on the fiber creates a moment which tends to cause the fiber to rotate (Martinie and Roussel 2011). When the fiber is aligned with the direction of flow, the drag-induced moment is zero.

Laranjeira et al. (2012) studied the contributions of mixing, casting, vibration, flow, and formwork geometry to the orientation of steel fibers in concrete. The effect of 
mixing depends on the rheological properties of the concrete: a stiff or unflowable mix will have an essentially random distribution of fiber orientations, whereas a flowable mix will tend to have the fibers aligned in a plane. Similarly, the degree of alignment introduced during casting depends on the flowability of the concrete and direction and height of placement. The height from which concrete is placed (i.e., how far it drops into the formwork) affects how much concrete placement disturbs concrete that is already in the form (Laranjeira et al. 2012). Findings by Laranjeira et al. (2012) for vibration and flow agreed with Edgington and Hannant (1972) and Martinie and Roussel (2011), respectively. Finally, formwork restricts the orientations that a fiber may take. Consider a fiber of length $\ell$ whose center is less than $\ell / 2$ from a wall. Such a fiber cannot take on very small angles (with respect to the wall's normal) without penetrating the wall, which of course cannot happen. The closer the fiber is to the wall, the greater the tendency to align in a plane parallel to the wall.

\subsubsection{Effect of orientation on behavior}

This section examines the effects of fiber orientation on strength for three loading types, tensile, compressive, and flexural.

\subsubsection{Tensile behavior}

The performance of fibers in a material under tension is probably the simplest and most widely-studied case. Fibers are most effective in carrying tensile stress when oriented parallel to the stress; perpendicular fibers do not contribute to load carrying ability but may restrain lateral movement due to Poisson effects (Krenchel 1964).

Krenchel (1964) derived an expression for the efficiency in tension of a composite with continuous fibers. For multiple groups of fibers, each oriented at an angle $\theta_{n}$ relative to the direction of loading, the efficiency is given by

where

$$
\eta=\sum_{n} a_{n} \cos ^{4} \theta_{n}
$$




$$
a_{n}=\frac{\text { vol } \% \text { of fiber group with } \theta_{n}}{\text { vol\% of all fibers }}
$$

For short-fiber composites (i.e., fibers with finite length), the efficiency of the fiber also depends on its length. The length $\ell_{S}$ required for full anchorage of the fiber is (Krenchel 1964):

$$
\ell_{s}=\frac{\sigma_{f}}{4 \tau_{o}} d
$$

where: $\quad \sigma_{f}=$ uniaxial tensile strength of fiber (psi);

$\tau_{o}=$ maximum bond strength between fiber and matrix (psi);

$d=$ fiber diameter (in.).

The efficiency modification for fiber length is

$$
\eta_{\ell}=1-2 \frac{\ell_{s}}{\ell}
$$

where $\ell$ is the actual length of the fiber (in.) (Krenchel 1964). Finally, the total efficiency factor, accounting for length and orientation, is

$$
\eta^{\prime}=\eta \eta_{\ell}
$$

Krenchel (1964) states that the initial elastic modulus varies with $\eta$ but that the ultimate strength is more likely to depend on the total efficiency $\eta^{\prime}$.

Pansuk et al. (2008) performed tension tests on UHPC specimens with different aspect ratios of fiber reinforcement. Fiber orientation was quantified using the orientation number $\eta$ and the orientation number multiplied by the fiber aspect ratio, $\eta(\ell / d)$. Results showed that both strength increase (relative to specimens with no fibers) and maximum crack opening increased with the two measures of fiber orientation, but there was not enough data to devise any empirical relationships.

Kang and Kim (2011) modeled the pre- and post-cracking behavior of UHPC tensile specimens, and experimentally investigated the effect of fiber orientation and fiber distribution. Dogbone specimens were cast so that fibers would be mainly aligned either 
parallel to the loading direction or perpendicular to the loading direction. The first cracking stress was about $10 \%$ higher for the parallel fibers. After cracks opened up, the fibers were engaged in crack bridging, and the effects of fiber orientation were more significant. The maximum stress was $40 \%$ higher for the parallel fibers.

Delsol and Charron (2013) reported on a series of tensile tests on UHPC with the goal of devising an empirical stress relationship as a function of fiber orientation. Orientations from 35 degrees to 54 degrees were observed in the specimens. The relationship was modeled as trilinear prior to peak stress; stress and strain at the key points were modeled using cosine functions of the form $a \cos \theta+b$, where $\theta$ is the orientation angle relative to the direction of pull and $a$ and $b$ are empirical constants. Three-point bending tests were also performed and the actual fiber orientation characterized after failure. Using the orientation and the empirical stress-strain relationship in a finite element model, Delsol and Charron (2013) were able to reproduce the experimental results fairly well, accounting for the \pm 2 degree error in the orientation measurement.

An empirical model for the increase in strength due to fibers was also proposed by Frettlöhr (2013), which adds an exponential term as well:

$$
\begin{aligned}
& \frac{\text { strength at orientation } \eta}{\text { strength with fibers perfectly aligned }}=\chi(\eta), \\
& \chi(\eta)=0.0014 \exp (5.97 \eta+0.22)+0.32 \eta
\end{aligned}
$$

where $\eta=\cos \theta$.

Finally, the Association Française de Génie Civil (AFGC) recommendations for UHPC (2013) specify the use of a K-factor to account for fiber orientation effects. When UHPC stress-strain properties for design are determined using tension tests, the tensile specimens may have more fibers aligned in the direction of loading than would be present in the actual structure. The K-factor is a reduction that is applied to the experimentallydetermined tensile strength to account for such unfavorable fiber orientations in the structure. Notably, the K-factor concept has been present since the recommendations 
were first issued in 2002, and application of the K-factor to several projects in France is surveyed by Simon et al. (2013).

\subsubsection{Compressive behavior}

An early work on compressive behavior was by Mansur et al. (1999), who investigated the effect of filling direction on high-strength concrete. The concretes that were studied had compressive strengths from $10 \mathrm{ksi}$ to $17 \mathrm{ksi}$. Prisms were cast standing upright (vertical) and laying on their sides (horizontal). Although fiber orientation was not directly measured, the filling and compaction process resulted in fiber alignment in the horizontal plane at the time of casting. After the specimens were cured, the prisms were tested standing upright. Thus, the prisms cast horizontally had fiber aligned in the direction of loading, whereas the prisms cast vertically had fibers aligned perpendicular to the direction of loading. The vertically cast prisms had higher peak stresses and strains at peak stress, as well as higher toughnesses, but the horizontally cast prisms had slightly higher initial tangent moduli.

Empelmann et al. (2008) investigated the effect of varying types of fibers on the post-peak compression behavior of UHPC. As might be expected qualitatively, adding fibers results in a more gradual descent curve than a no-fiber reference. Also, specimens with two or more types of fibers, particularly combinations of steel fiber with polypropylene "microfibers," further increased the area under the post-peak stress-strain curve.

Leonhardt et al. (2012) performed impact testing on UHPC. Specimens were preloaded with $1 \mathrm{kN}(225 \mathrm{lbf})$, and a $50-\mathrm{kg}(110-\mathrm{lbm})$ weight was dropped from a height of $0.6 \mathrm{~m}(1.97 \mathrm{ft})$ five times. The ultrasonic pulse velocity was measured, and the process of five drops followed by pulse velocity measurement was repeated until the measured pulse velocity was $90 \%$ of the initial value. This was designated as failure. Leonhardt et al. (2012) observed that most cracks formed longitudinally, indicating a tensile stress perpendicular to the impact loading (due to Poisson effects). After failure, the cylinders were cut into three transverse slices and the fiber orientation in the horizontal and vertical directions was measured using electrical induction equipment. Factors resulting in better 
resistance to impact were fiber content, uniformity of fiber distribution, and percentage of fibers oriented horizontally.

VanSlembrouck (2015) noted that fiber alignment seemed to have a strong influence on the dynamic compressive behavior of Cor-Tuf. The observed failure modes were classified according to ASTM C39/C39M guidelines (ASTM 2015). The majority of specimens exhibited either type 2 failures, with a well-formed cone on one end only and vertical cracks on the other, or type 4 failures, with a diagonal fracture and no end cracks (VanSlembrouck 2015). Qualitative observations of the fiber orientation were also made. Based on visual inspection, the fibers in the failure surface were classified as aligned in the failure plane, aligned in a horizontal plane, or other. The other category includes orientations that appeared random, though it was not possible to determine if they were truely random from visual observations. Of the 36 specimens with type 2 or 4 failures, about 31\% had fibers aligned in the plane of failure (VanSlembrouck 2015).

\subsubsection{Flexural behavior}

Kim et al. (2008) investigated the effect of placement direction on fiber alignment and mechanical performance of UHPC beams. Two casting directions were used: parallel and perpendicular to the beam's longitudinal axis. Using optical characterization on cut sections, it was found that fibers tended to align in the direction of casting. The beams were tested under 4-point bending with a $250-\mathrm{kN}$ (56-kip) loader operating in displacement control, monitoring midspan deflection using an LVDT. The first cracking loads were roughly the same, but the ultimate load was $50 \%$ higher for parallel placement.

Barnett et al. (2010) tested round slabs cast from the center, edge, and randomly. Strangely, fibers were observed to align perpendicular to the flow of fresh concrete. Panels poured from the center were found to provide the greatest load capacity. Because the slab's collapse mechanism involved radial cracking, fibers oriented in the hoop direction were most efficient in bridging the cracks.

Trainor et al. (2013b) performed 3-point bending tests using CMOD-control on Cor-Tuf beams and characterized the fiber orientation post-failure using $\mathrm{x}$-ray CT. 
Results showed that fiber volume fraction had no clear effect on peak load or net work of load, but that fiber orientation played a significant role.

\subsection{SHPB Testing}

\subsubsection{Historical development}

Hopkinson (1914) first used the motion of an elastic bar as a means of measuring pressures. Kolsky (1949) modified this technique for dynamic compression testing of rubbers, plastics, and metals by using two bars with a specimen placed between them. Transient stress waves were induced by an explosion, and specimen stress and strain were determined from strain measurements on the bars. The use of elastic wave propagation theory for the calculation of stress and strain will be covered in Section 2.3.2. In recognition of Kolsky's work, the split-Hopkinson pressure bar (SHPB) is sometimes also referred to as a Kolsky bar.

Krafft et al. (1954) made modifications that are commonly used in modern SHPB designs: using strain gauges for measuring strain in the bars rather than the condenser microphones used previously, and using a striker bar fired from a gun to produce a transient stress wave (Chen and Song 2011). A schematic of a modern SHPB is shown in Figure 2.3. On this SHPB, a compressed gas cannon is used to launch the striker bar, and a chronograph is used to measure the velocity of the striker bar. The specimen is placed between the input bar and the output bar, which are both instrumented with strain gages. Signals from strain gages on these bars are fed through a bridge amplifier and meter (BAM) unit to convert resistance changes in the gages to an output voltage (using a Wheatstone bridge), and then amplify that voltage. Voltage signals may be recorded using an oscilloscope, or PC-based oscilloscope with an analog-to-digital converter (Gilbertson 2011). The stop bar captures the momentum of the output bar. 


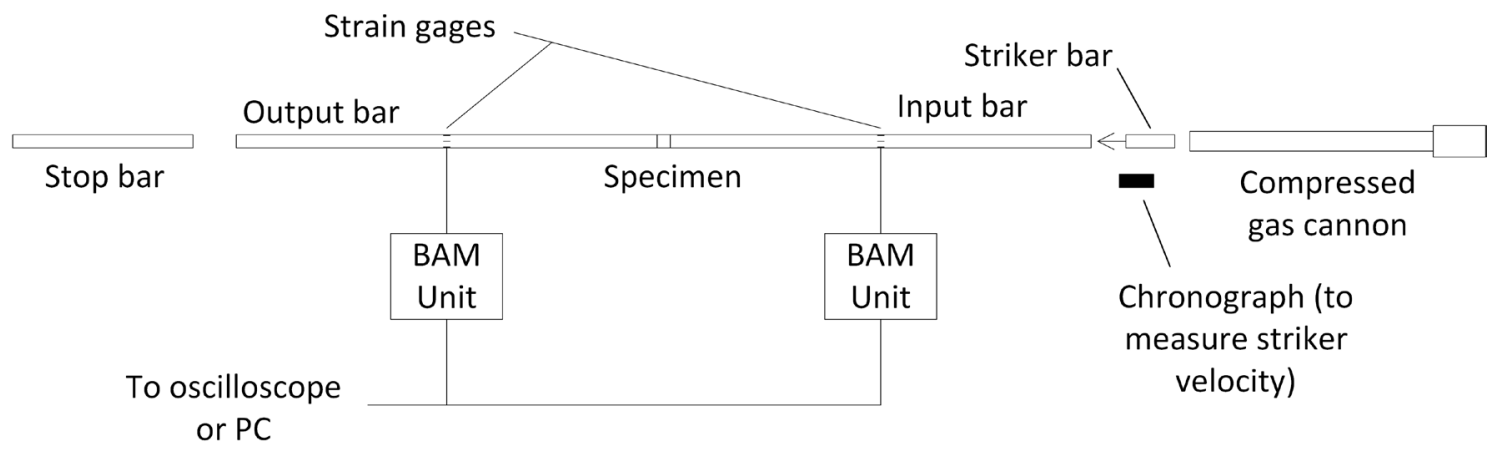

Figure 2.3. The split-Hopkinson pressure bar.

Davies and Hunter (1963) used the SHPB for dynamic compression testing of metals, polymers, and rubber. Lindholm and Yeakley (1968) performed a review of progress in SHPB compression testing techniques, and devised a method of performing tension tests as well by using a hat-shaped specimen.

By placing a deformable material, called a "pulse shaper," between the striker bar and input bar, it is possible to alter the stress pulse that is propagated through the bar and into the specimen. Frew et al. $(2001 ; 2002)$ used thin copper discs to produce a ramp pulse which resulted in an approximately constant strain rate and approximate dynamic stress equilibrium in rock and glass ceramic specimens. Pulse shapers are covered in more detail in Section 2.3.4.

\subsubsection{Elastic wave propagation theory}

The SHPB apparatus is designed so that the bars will remain elastic. This permits indirect measurement of stress and strain at the specimen-bar interface by measuring strain in the bar. Because strain measurements are often taken at the bar midpoint, wave dispersion may alter the measured strain and can be corrected for (see Section 2.3.5). One-dimensional elastic wave theory is typically used for analyzing SHPB data because of its simplicity, though two-dimensional axisymmetric and three-dimensional theories have also been considered.

One-dimensional elastic wave theory can be derived from Newton's second law and Hooke's law; the derivation can be found in many works, such as Jerome (1991). Applied to the SHPB, this theory assumes wave propagation is uniform across the bar's 
cross section, and only varies along the length. Assumptions inherent in using the theory will be considered in detail in Section 2.3.6. The governing equation for one-dimensional elastic wave theory is

$$
\frac{\partial^{2} u}{\partial t^{2}}=c_{0, b}^{2} \frac{\partial^{2} u}{\partial x^{2}}
$$

where: $\quad u=$ longitudinal displacement (in.);

$t=$ time $(\mathrm{s})$

$c_{0, b}=$ elastic wave speed of the bar, $\sqrt{E_{b} / \rho_{b}}$ (in./s);

$E_{b}=$ elastic modulus of the bar (psi);

$\rho_{b}=$ mass density of the bar (lbm/in. $\left.{ }^{3}\right)$;

$x=$ longitudinal coordinate (in.).

Expressions may be derived from equation (2.10) for the displacement and velocity at the specimen ends, and thus the strain and strain rate. This has been done by many authors, for example, Lindholm and Yeakley (1968). The average strain $\varepsilon_{s}$ and strain rate $\dot{\varepsilon}_{S}$ in the specimen are

$$
\begin{gathered}
\varepsilon_{S}(t)=\frac{c_{0, b}}{L_{S}} \int_{0}^{t}\left(\varepsilon_{I}(\tau)-\varepsilon_{R}(\tau)-\varepsilon_{T}(\tau)\right) d \tau \\
\dot{\varepsilon}_{S}(t)=\frac{c_{0, b}}{L_{S}}\left(\varepsilon_{I}(t)-\varepsilon_{R}(t)-\varepsilon_{T}(t)\right)
\end{gathered}
$$

where: $\quad L_{S}=$ length of undeformed specimen (in.);

$\varepsilon_{I}=\quad$ strain from incident stress pulse (in./in.);

$\varepsilon_{R}=\quad$ strain from reflected stress pulse (in./in.);

$\varepsilon_{T}=\quad$ strain from transmitted stress pulse (in./in.).

With the input bar-specimen interface denoted by 1 and the specimen-output bar interface denoted by 2 , the forces $P_{1}$ and $P_{2}$ on the specimen ends are (Lindholm and Yeakley 1968): 


$$
\begin{aligned}
& P_{1}(t)=A_{b} E_{b}\left(\varepsilon_{I}(t)+\varepsilon_{R}(t)\right), \\
& P_{2}(t)=A_{b} E_{b}\left(\varepsilon_{T}(t)\right)
\end{aligned}
$$

where $A_{b}$ is the area of the bar (in. ${ }^{2}$ ) and the input and output bars are assumed to have the same properties. If desired, the stresses on the specimen ends are simply $\sigma_{1}=P_{1} / A_{S}$ and $\sigma_{2}=P_{2} / A_{s}$. The average stress in the specimen is, therefore,

$$
\sigma_{s}(t)=\frac{P_{1}(t)+P_{2}(t)}{2 A_{s}}=\frac{A_{b} E_{b}}{2 A_{s}}\left(\varepsilon_{I}(t)+\varepsilon_{R}(t)+\varepsilon_{T}(t)\right)
$$

where $A_{s}$ is the area of the undeformed specimen (in. ${ }^{2}$ ). "One-wave" analysis uses the simplification $\varepsilon_{I}(t)+\varepsilon_{R}(t)=\varepsilon_{T}(t)$, allowing the "three-wave" equations above to be written in terms of a single strain. This approximation is only valid if the specimen is in force equilibrium, as can be seen from equation (2.13).

Davies and Hunter (1963) performed experiments with the SHPB on metal, rubber, and polymer specimens, and analyzed the contribution of inertia to the apparent stress. Through a one-dimensional analysis, it was found that

$$
\sigma_{a}(t)=\sigma_{o}(t)+\rho_{s} \beta^{2}\left(\frac{1}{6} L_{s}^{2}-\frac{1}{8} v_{s}^{2} D_{s}^{2}\right) \frac{\partial^{2} \varepsilon_{s}(t)}{\partial(\beta t)^{2}}
$$

where: $\quad \sigma_{a}=$ apparent stress (psi);

$\sigma_{o}=$ actual stress (psi);

$\rho_{s}=$ mass density of specimen $\left(\mathrm{lbm} / \mathrm{in} .^{3}\right)$;

$\beta=$ parameter for wave dispersion correction $\approx 1$ (unitless);

$v_{s}=$ Poisson's ratio of specimen (unitless);

$D_{S}=$ diameter of undeformed specimen (in.).

Rand (1967) performed a one-dimensional analysis of friction in the SHPB, building on the work of Jackson and Waxman (1963). For an incompressible specimen obeying the Tresca yield criterion and the Hencky-Mises flow law, Rand (1967) showed that the ratio of apparent stress to actual stress was 


$$
\begin{aligned}
& \frac{\sigma_{a}(t)}{\sigma_{o}(t)}=\frac{2}{(\alpha(t))^{2}}[\exp (\alpha(t))-\alpha(t)-1], \\
& \alpha(t)=\frac{\mu}{L_{S} / D_{S}}\left(1+\varepsilon_{S}(t)\right)^{-3 / 2}
\end{aligned}
$$

where $\mu$ is the coefficient of friction (unitless).

Bertholf and Karnes (1975) carried out a two-dimensional numerical study of wave propagation, and examined frictional and inertial effects on one-dimensional predictions. It was found that the apparent increase in stress due to friction varied with $\mu /\left(L_{s} / D_{s}\right)$, consistent with, though smaller than, Rand's (1967) prediction. Also, the simulations of Bertholf and Karnes (1975) confirmed the general form of the inertial correction from Davies and Hunter (1963). A three-dimensional elastic wave theory was arrived at independently by Pochhammer (1876) and Chree (1889), but is too complex mathematically to be within the scope of the present work. Pochhammer-Chree theory was applied by Jerome (1991) in analyzing a 6-in. diameter SHPB, under the assumption that only axisymmetric vibration modes would occur, allowing treatment in two dimensions.

\subsubsection{Specimen preparation}

Specimens for SHPB testing are typically cylindrical, with varying aspect ratios $L_{s} / D_{s}$. Davies and Hunter (1963) derived equation (2.15) for the contribution of inertia to the measured stress. Based on this, an ideal aspect ratio was determined to cancel out the inertial term. This ratio is

$$
\frac{L_{s}}{D_{s}}=\frac{\sqrt{3}}{2} v_{s} .
$$

For materials with $v_{s} \leq 0.5$, the equation calls for aspect ratios of 0.433 or less. In general, as the aspect ratio decreases, the effect of axial inertia decreases, but friction and radial inertia become more prominent. As shown by Rand (1967), friction between the bars and the specimen also introduces error. Typically, the coefficient of friction at the 
specimen-bar interface is reduced by lubrication with $\mathrm{MoS}_{2}$, Teflon, or another dry film lubricant. When the coefficient of friction is less than 0.1 , finite element modeling suggests that friction's effect on the measured failure strength is negligible (Q. M. Li and Meng 2003).

The aspect ratios used in SHPB testing vary widely. As a rule of thumb, it is suggested that an aspect ratio between 0.5 and 1 be used, as a sort of compromise between frictional and inertial effects (Gray III 2000). Clark (2013) performed a literature review on aspect ratios and found that a majority of tests on concrete were conducted with aspect ratios of 0.5 or 1, with a few researchers using 2. Clark (2013), and later VanSlembrouck (2015), used aspect ratios of 0.5 and 1 in their work, with some specimens at 2 for comparison. VanSlembrouck (2015) did not find a statistically significant difference in failure strength among the three aspect ratios. However, as predicted by equation (2.12), the maximum attainable strain rate does decrease with increasing specimen length.

It is also important that the ends of the specimen are flat and parallel. Gray III suggests the surfaces be flat within 0.001 in., or even 0.0001 in. for brittle materials (2000). For parallelness of Ductal ${ }^{\circledR}$ specimens, the suggested tolerance is 0.5 degrees (Lafarge North America). Clark (2013) found that specimens outside the recommended parallelness tolerance exhibited considerably more scatter in dynamic compressive strength than specimens meeting the tolerance.

\subsubsection{Stress pulse and pulse-shaping}

An incident stress pulse is generated in the input bar by the impact of the striker bar. The theoretical amplitude $\sigma_{I}$ is determined by the velocity of the striker (Chen and Song 2011):

$$
\sigma_{I}=\frac{1}{2} \rho_{b} c_{0, b} V_{s t}
$$

where $V_{s t}$ is the striker velocity (in./s). Equation (2.18) can be divided by $E_{b}$ and simplified to give the theoretical strain in the bar: 


$$
\varepsilon_{I}=\frac{V_{s t}}{2 c_{0, b}}
$$

The duration $T$ of the pulse is twice the wave transit time in the striker, and hence proportional to the striker's length:

$$
T=2 \frac{L_{s t}}{c_{0, s t}}
$$

where: $\quad L_{s t}=$ length of striker bar (in.);

$$
c_{0, s t}=\text { elastic wave speed of the striker, } \sqrt{E_{s t} / \rho_{s t}} \text { (in./s). }
$$

Without pulse shaping, the stress pulse is essentially a square wave, with some Pochhammer-Chree oscillations due to wave dispersion (Chen and Song 2011). For brittle materials, the goal of using a pulse shaper is to (1) reduce stress non-equilibrium and (2) achieve a more constant strain rate. Stress non-equilibrium is reduced by increasing the rise time of the incident pulse (Frew et al. 2001; 2002; Zhang et al. 2009), resulting in a ramp pulse instead of a square pulse. A constant strain rate is very difficult to achieve, but it is possible to limit the strain acceleration so the strain rate does not vary excessively (Zhang et al. 2009).

The material and geometry of the pulse shaper depends on the material to be tested. Clark (2013) reviewed pulse shapers used in previous work and concluded that, for brittle materials, pure copper was most commonly used. Four sizes of C1100 copper pulse shaper discs were tested, and 0.75-in. diameter by 0.085-in. thick discs were ultimately selected (Clark 2013). The same pulse shaper was also used by VanSlembrouck (2015). Rubber ring pulse shapers have also been used for testing mortar (Zhang et al. 2009).

\subsubsection{Data processing}

Without going into the details of the procedures used (which are described in Section 3.5), this section will cover the basics of SHPB data processing. Voltages are recorded from strain gages, typically located at the middle of each bar. The strain gages are usually connected in a half- or full-Wheatstone bridge to cancel out bending effects. 
Strains are calculated from the voltages, and these strains are used with the equations presented in Section 2.3.2 to calculate the stress, strain, and strain rate.

Dispersion affects the wave as it travels down the bar, although this dispersion is not accounted for in one-dimensional elastic wave propagation theory. If wave dispersion effects are to be corrected for, Pochhammer-Chree theory must be used. Follansbee and Frantz (1983) presented a method for correcting dispersion effects using Pochhammer-Chree theory that enables the one-dimensional theory to be used for analyzing the data after the correction. The method uses a Fourier transform to take the strain pulses from the time domain into the frequency domain. According to Pochhammer-Chree theory, the propagation velocity decreases as frequency increases; the various frequency components of the waveform are adjusted to account for this velocity variation. Finally, an inverse Fourier transform is used to return the waveform to the time domain.

\subsubsection{Conditions for validity}

For the 1-D wave propagation theory to be applicable to analyzing an SHPB experiment, the following criteria should be met (Chen et al. 1994; Gama et al. 2004):

(1) The bar remains elastic.

(2) The stress pulse is uniform across the cross-section of the bar and is not affected by dispersion.

(3) The bar-specimen interfaces remain flat and parallel (no indentation).

(4) The effects of friction at the bar-specimen interfaces are negligible.

(5) The stress distribution in the specimen is uniform, both axially and radially.

(6) The effects of radial and axial inertia in the specimen are negligible.

Assumption 1 may be met by limiting the striker velocity. According to Gray III (2000), assumption 2 is approximately satisfied when the bar length is greater than ten times the bar diameter. Assumption 3 will be satisfied if the specimen does not indent into the bar (Chen et al. 1994), which can be avoided by using specimens with the same diameter as the bar. Assumption 4 is generally addressed by lubrication. It is doubtful 
whether stress equilibrium (assumption 5) can be perfectly achieved, but it has been suggested (Ravichandran and Subhash 1994) that it may be assumed if

$$
\left|\frac{\Delta \sigma(t)}{\sigma_{m}(t)}\right|=2\left|\frac{\sigma_{1}-\sigma_{2}}{\sigma_{1}+\sigma_{2}}\right| \leq 0.05
$$

where $\Delta \sigma(t)$ is the stress difference between the two ends (psi) and $\sigma_{m}(t)$ is the mean stress (psi). Stress equilibrium is addressed in more detail in Section 2.3.7. Finally, assumption 6 may or may not be accurate, but accounting for inertial contributions to the measured stresses and to the material response would likely require numerical modeling.

\subsubsection{Stress equilibrium}

Achieving a state of uniform stress throughout the specimen is a major concern for a valid SHPB test. Davies and Hunter (1963) performed a theoretical analysis of stress equilibrium to assess the validity of SHPB results. The propagation of plastic disturbances in a deforming metal is described by Taylor-von Karman theory, which lead Davies and Hunter (1963) to the following criterion for the slope of the stress strain curve:

$$
\frac{\mathrm{d} \sigma_{s}}{\mathrm{~d} \varepsilon_{s}}>\frac{\pi^{2} \rho_{s} L_{s}^{2}}{T^{2}}
$$

where all terms have been previously defined. Davies and Hunter (1963) noted that this may be interpreted to mean that the loading pulse time $T$ should be greater than the time required for roughly three disturbances to propagate across the specimen. The time $\tau$ required for an elastic wave to cross the length of the specimen is

$$
\tau=\frac{L_{s}}{c_{0, s}}
$$

where $c_{0, s}$ is the elastic wave speed of the specimen, $\sqrt{E_{s} / \rho_{s}}$ (in./s).

Yang and Shim (2005) analyzed the number of wave transits in the specimen required to satisfy inequality (2.21) in terms of the relative acoustic impedance $\beta$. For a 
specimen loaded by a stress pulse which reaches constant magnitude after a rise time $2 \tau$, the right side of inequality (2.21) is given by

$$
\left|\frac{\Delta \sigma}{\sigma_{m}}\right|=\frac{2 \beta^{2}(1-\beta)^{k-2}}{(1+\beta)^{k}-(1-\beta)^{k-2}}
$$

where the equation is only valid for a number of wave transits $k>2$, and the relative acoustic impedance is

$$
\beta=\frac{A_{s} \rho_{s} c_{0, s}}{A_{b} \rho_{b} c_{0, b}} .
$$

If the specimen is loaded by a linearly-increasing pulse and fails within the rise time, the solution given by Yang and Shim (2005) is instead

$$
\left|\frac{\Delta \sigma}{\sigma_{m}}\right|=\frac{2 \beta^{2}\left[1-\left(-\left(\frac{1-\beta}{1+\beta}\right)\right)^{k}\right]}{2 k \beta-1+\left(\frac{1-\beta}{1+\beta}\right)^{k}}
$$

where the equation is again valid only for a number of wave transits $k>2$.

\subsection{Dynamic Compressive Behavior of UHPC}

Both normal-strength concrete (Bischoff and Perry 1991; Ross et al. 1995) and UHPC (Cavill et al. 2006; Jiao et al. 2009; Lai and Sun 2009; Zhang et al. 2009; Ju et al. 2010; Rong et al. 2010) show strength increases at high strain rates. This strength increase is customarily quantified using the dynamic increase factor (DIF), the ratio of dynamic failure strength to quasistatic failure strength. This section will concentrate on aspects of strain rate-sensitivity specific to UHPC, and some recent developments on the effect of fibers will be discussed.

Othman and Marzouk (2016) investigated the dynamic behavior of two FRCs and a high-strength concrete (HSC) at moderate strain rates of $3 \times 10^{-5} \mathrm{~s}^{-1}$ to $0.1 \mathrm{~s}^{-1}$, which span quasistatic and vehicle impact loading regimes. These materials are not, strictly speaking, UHPCs, but do have some similarity in their dynamic response. The FRC 
quasistatic compressive strengths (16 ksi and $19 \mathrm{ksi}$ ) approach the $22 \mathrm{ksi}$ threshold for UHPC, but the HSC quasistatic compressive strength of $12 \mathrm{ksi}$ was far below the threshold. Remember that strength is not the only criterion for a UHPC, however. The DIF at the highest strain rate, $0.1 \mathrm{~s}^{-1}$, was $1.08-1.09$ for the FRCs and 1.14 for the HSC. This illustrates the trend of lower compressive DIFs for stronger materials. Also, a matrix without fiber reinforcement generally has a higher DIF than a matrix with fiber reinforcement (Millard et al. 2010).

$\mathrm{Yu} \mathrm{Su}$ et al. (2016) investigated the effect of various fiber types on dynamic compressive strength of UHPC. Four types of fibers were considered, 0.12-mm (0.0047in.) diameter microfibers with lengths of $6 \mathrm{~mm}$ and $15 \mathrm{~mm}$ (0.24 in. and $0.59 \mathrm{in}$.), and $0.3-\mathrm{mm}$ and $0.5-\mathrm{mm}(0.012-\mathrm{in}$. and 0.020 -in.) diameter twisted fibers with lengths of 30 $\mathrm{mm}$ (1.2 in.). Note that the microfibers had a tensile strength of $4295 \mathrm{MPa}(623 \mathrm{ksi})$ whereas the twisted fibers had a tensile strength of $1500 \mathrm{MPa}(218 \mathrm{ksi})$. For a given fiber type and fiber volume fraction, quasistatic compressive strength increased with fiber aspect ratio, but strain at peak stress was higher for lower fiber aspect ratios. Dynamic tests performed at $50-100 \mathrm{~s}^{-1}$ indicated that micro fibers provided superior performance, due, at least in part, to their increased tensile strength. The largest DIF, about 1.8, was achieved with 6-mm microfibers, and the largest dynamic compressive strength, about $220 \mathrm{MPa}$ (32 ksi), was achieved with $15-\mathrm{mm}$ microfibers. The trend of increasing strength with increasing fiber aspect ratio was seen at dynamic rates as well; strain at peak stress varied less with fiber aspect ratio than it did in quasistatic testing.

A central question is whether the strength increase in dynamic tests represents rate-sensitivity of the material or confinement effects due to the test method and specimen. Physical factors for the increase in strength at high strain rates include matrix viscoelasticity (Q. M. Li and Meng 2003) and reduced time for microcrack propagation (Q. M. Li and Meng 2003; Jiao et al. 2009). The strength of concrete is also affected by the confining pressure, as shown by the Drucker-Prager model (Drucker and Prager 1952), for example. Because of the short duration of loading in SHPB tests, there is concern that friction or radial inertia may serve to increase the confinement on the specimen during testing. Q. M. Li and Meng (2003) used a rate-insensitive Drucker- 
Prager model in ABAQUS/Explicit to model SHPB tests of concrete. It was found that the hydrostatic stress was not negligible, and seemed to be responsible, at least in part, for the strength increase. The model shows that radial confinement effects become significant for strain rates of about $10^{2} \mathrm{~s}^{-1}$ and above.

Zhang et al. (2009) tested tubular and solid mortar specimens seeking to determine what effect confinement had on concrete response in the SHPB. The tubular specimens had a cylindrical hole in the middle to reduce the amount of material and, hence, the amount of confinement. Results showed that solid specimens that were not pulverized had an intact central core, consistent with confinement at the center providing increased strength. At strain rates of $50-300 \mathrm{~s}^{-1}$, the tubular specimens had a DIF up to $14 \%$ less than solid specimens. Due to this evidence of confinement effects, numerical simulation was recommended to back out the uniaxial stress response from the test data. Zhang et al. (2009) did not analyze whether stress concentrations could have played a role in the results, however.

Recall the discussion of apparent stress and actual stress in Section 2.3.2. A more detailed treatment of these additional stresses in a cylindrical coordinate system was given by Forrestal et al. (2007) using linear elasticity theory. For both compressible and incompressible specimens, the additional radial stress has a parabolic distribution, greatest at the center and decreasing toward the edges. For a compressible specimen, the radial stress at a distance $r$ from the center is shown below:

$$
\sigma_{r}=\frac{v_{s}\left(3-2 v_{s}\right)}{8\left(1-v_{s}\right)}\left(\frac{D_{s}^{2}}{4}-r^{2}\right) \rho_{s} \ddot{\varepsilon}_{s}
$$

where $\ddot{\varepsilon}_{S}$ is the strain acceleration, $\mathrm{d}^{2} \varepsilon_{S} / \mathrm{d} t^{2}\left(\mathrm{~s}^{-2}\right)$.

Note that radial inertia will also cause an increase in axial stress (Forrestal et al. 2007). This artificial increase in axial stress can be accounted for, but correcting for the effect of radial confinement on the material response would require a material model for multiaxial stress under dynamic loading. 


\subsection{X-Ray Computed Tomography}

\subsubsection{Equipment}

At its most basic, $\mathrm{x}$-ray $\mathrm{CT}$ requires an $\mathrm{x}$-ray source, a detector, and a means of moving the specimen (or the x-ray source-detector assembly) to obtain different paths through the specimen. Tube $\mathrm{x}$-ray sources are commonly used, consisting of an electron accelerator and target material (Landis and Keane 2010). The accelerated electrons collide with the target to produce $\mathrm{x}$-ray photons with a spectrum of energies (Bremsstrahlung radiation) and a large number of $\mathrm{x}$-ray photons at a few specific energies (characteristic emissions) which depend on the element used for the target (Buzug 2008).

Detection of $x$-rays is accomplished through two steps: scintillation, where impinging x-rays cause the emission of visible light, and photodetection, where the visible light is recorded by an array of photodetectors (Landis and Keane 2010).

As discussed in the next section, the principle behind $\mathrm{x}$-ray $\mathrm{CT}$ is that, by measuring the total attenuation of x-rays on different paths through a specimen, the internal distribution of attenuation can be reconstructed. Measuring these different paths requires rotating the specimen (or x-ray source-detector assembly). For industrial applications, a rotating stage is used to rotate the specimen, whereas medical applications rotate the source-detector assembly to avoid disturbing the patient (Goldman 2007).

\subsubsection{Underlying principles}

As an x-ray beam travels through a material, its intensity is reduced through attenuation. If the material is homogeneous, the attenuation is described by the Beer-Lambert law (Buzug 2008), given below:

$$
\frac{I}{I_{0}}=e^{-(\mu / \rho) \rho x}
$$

where: $\quad I / I_{0}=$ ratio of final intensity to initial intensity;

$\mu / \rho=$ mass attenuation coefficient (in. ${ }^{2} / \mathrm{lbm}$ );

$\mu=$ attenuation coefficient (1/in.);

$\rho=$ mass density $\left(\mathrm{lbm} / \mathrm{in} .{ }^{3}\right)$; 


$$
x=\text { specimen thickness (in.). }
$$

When imaging a specimen, the material is not homogeneous, and the attenuation of an x-ray beam is instead determined by the total attenuation of all the material it encounters along its path. If the total attenuation is defined as $\tau=(\mu / \rho) \rho x$ (Landis and Keane 2010), then the attenuation may be determined from

$$
\tau=\ln \frac{I_{0}}{I}
$$

In practice, the detector cannot be assumed to give a zero reading for zero radiation, so a calibration reading is taken before imaging a specimen. The dark field intensity $I_{d}$ is the detector reading corresponding to no radiation, and may vary at different points on the detector (Landis and Keane 2010). The corrected attenuation is then

$$
\tau=\ln \frac{I_{0}-I_{d}}{I-I_{d}}
$$

Determining the distribution of attenuation within the specimen based on the attenuation of different paths is an inverse problem. The attenuation of a path can be represented as a line integral of attenuation along the path through the specimen. These line integrals are also referred to as projections or projection functions. Radon provided a theoretical basis for reconstructing a 2-D function based on line integrals of the function (Radon 1917; translated in Radon 1986). Although mathematically sound, Radon's approach presents practical difficulties due to errors in the acquired data (Landis and Keane 2010). Several other reconstruction algorithms have been used since medical xray CT was introduced by Hounsfield in 1971. Hounsfield originally divided the sample into a grid of cells, each with an unknown attenuation (Goldman 2007). Because the attenuation on a path is the sum of the attenuations of the cells on the path, the determination of the unknowns is simple, if computationally intensive, linear algebra.

Modern reconstruction techniques include filtered backprojection (FBP) and direct Fourier inversion (DFI) (Landis and Keane 2010). FBP consists of two steps: backprojection, which allows fast reconstruction but produces blurry images, and filtering 
to sharpen the resulting image. Backprojection works by equally distributing attenuation along each path. Summing a number of these backprojections taken from different angles produces a reconstructed image, albeit with significant blurriness and some artifacts (Goldman 2007). Filtering, or convolution, increases the sharpness of edges and other features at the expense of increasing noise (Goldman 2007). DFI is based on the Fourier projection slice theorem, also called the central section theorem. Broadly speaking, the Fourier projection slice theorem states that the 1-D Fourier transform of a projection is equivalent to the 2-D Fourier transform of the slice that the projection passes through (Landis and Keane 2010).

Because of the competitive value of efficient reconstruction algorithms, many are proprietary (Landis and Keane 2010). Therefore, researchers using commercial x-ray CT systems typically do not have access to the details of the algorithms used to reconstruct the 3-D distribution of attenuation within test specimens. This reconstruction results in what may be considered raw image data, which is then operated on with image processing techniques to yield information about the internal structure of the specimen.

\subsubsection{Application to cementitious materials}

Martz et al. (1993) demonstrated the applicability of CT to imaging the internal structure of concrete specimens. Gamma-ray, rather than x-ray, CT was used to image a cylinder with a conical void and to determine the distribution of rebar inside a cube, demonstrating qualitatively the possibilities of CT.

X-ray CT has been used by a number of researchers, particularly for determining fiber orientation (Schnell et al. 2008; Barnett et al. 2010; Krause et al. 2010; Trainor et al. 2013b; Oesch 2015), though it is capable of characterizing void and aggregate distribution as well (Yu-Min Su 2012; Oesch 2015). The phases in the concrete-mortar, aggregate, fiber, and voids - can be distinguished based on their attenuation values. The mortar and aggregate typically have similar attenuation values; steel fibers and voids are well-separated from the mortar/aggregate, having much higher and lower attenuations, respectively (Oesch 2015). 
Oesch (2015) performed unconfined compression and double-punch tensile tests on NSC and Cor-Tuf specimens. Specimens were loaded incrementally and CT scanned after each load increment to observe cracking and damage behavior. Unconfined compression tests were conducted in four load increments, loading to $75 \%, 85 \%, 95 \%$, and finally $100 \%$ of ultimate strength (Oesch 2015). Double-punch tests were also conducted in four load increments, loading to $95 \%$ and $100 \%$ of ultimate strength, followed by loading to $150 \%$ and then $200 \%$ of displacement at ultimate strength (Oesch 2015). Results showed that fiber orientation within the specimens was not random; the specimens were cored from a slab, and the observations were consistent with fibers aligning in the flow direction when the slab was cast (Oesch 2015). Fiber orientation was also found to influence the cracking pattern observed in the double-punch tests (Oesch 2015), which differed from the pattern that is typically assumed in analyzing these tests (Molins et al. 2009).

Oesch (2015) also used CT scanning to evaluate damage to Cor-Tuf specimens subjected to fragment-simulating projectile loads in research conducted by Scott et al. (2015), and damage to Cor-Tuf specimens in rebar pull-out experiments. In the pull-out experiments, Grade 60, No. 3 bars were embedded roughly 4.75 in. into 3 in. by 6 in. Cor-Tuf or NSC cylinders (Oesch 2015). In the NSC tests, the concrete failed around the rebar, whereas the Cor-Tuf tests exhibited tensile rupture of the rebar some distance above the top of the concrete (Oesch 2015). 


\section{CHAPTER 3 METHODS}

\subsection{Specimen preparation}

Specimens were prepared at the Geotechnical and Structures Laboratory (GSL) of the U.S. Army Engineer Research and Development Center (ERDC) in June and July 2015.

\subsubsection{Formwork construction}

Formwork for the beam was constructed using 3/4-in. BC-finish plywood supported by $2 \times 4$ lumber. The form was designed to accommodate a beam measuring $10 \mathrm{ft}$ long, $2 \mathrm{ft}$ high, and 8 in. wide. All joints, as well as knots in the plywood, were filled with putty. The form was made more sturdily than is typical as it needed to be moved from the shop to the batch plant. The bottom of the form consisted of plywood supported on skids made of landscape timbers. This formed what was essentially a pallet so that the beam could be moved easily after demolding. The completed form is shown in Figure 3.1.

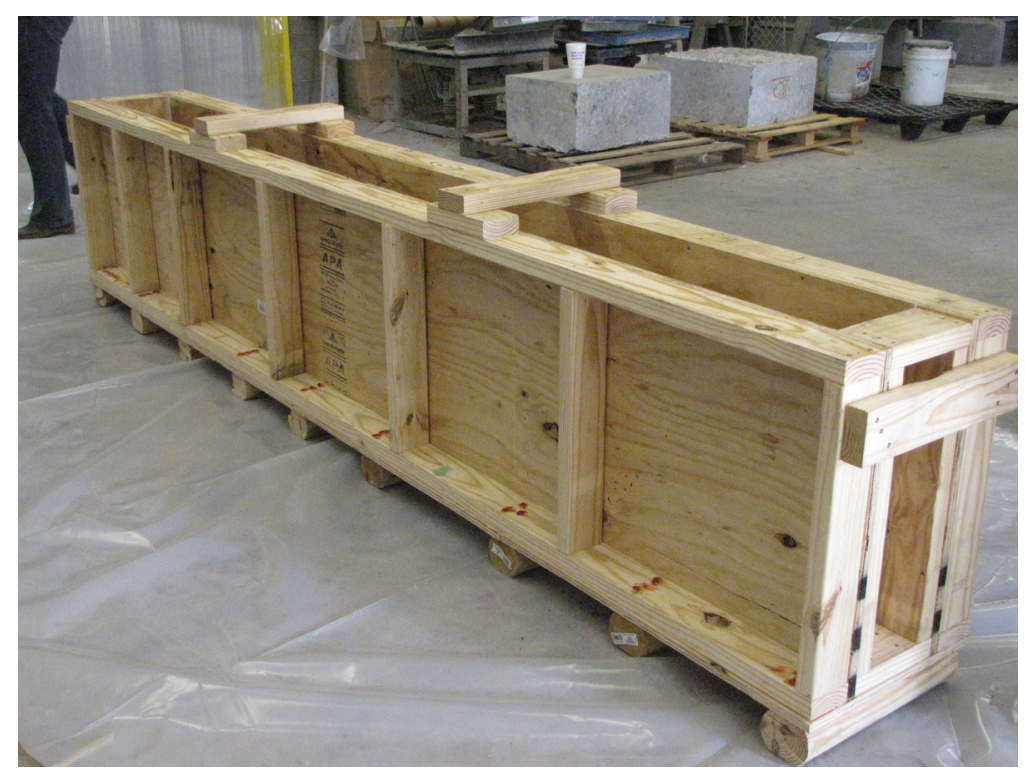

Figure 3.1. Completed formwork for beam. 


\subsubsection{Mixture proportions}

A UHPC developed by the U.S. Army Corps of Engineers called Cor-Tuf (Williams et al. 2009) was used for this research. A standard mixture for Cor-Tuf with steel fibers (CT-F) was used, incorporating $3.15 \mathrm{vol} \%$ fiber reinforcement. The mixture proportions are given below in Table 3.1.

Table 3.1. Cor-Tuf mixture proportions.

\begin{tabular}{|c|c|c|}
\hline Material & Description & Weight (lbf) \\
\hline Cement & Class H cement, Quality Stone and Readymix & 836.2 \\
\hline Silica Fume & Elkem ES900W & 325.9 \\
\hline Sil co Sil & U.S. Silica & 231.4 \\
\hline Silica Sand & U.S. Silica F-50 & 811.0 \\
\hline Steel fibers & Bekaert Dramix ZP 305 & 262.4 \\
\hline Superplasticizer & W.R. Grace ADVA 190 & 14.29 \\
\hline \multirow[t]{3}{*}{ Water } & Vicksburg, MS municipal water supply & 174.3 \\
\hline & Total weight (lbf) & 2655.5 \\
\hline & Batch size $\left(\mathrm{ft}^{3}\right)$ & 17 \\
\hline
\end{tabular}

\subsubsection{Mixing}

The mixing was performed in a Nikko SF 1000 HD Twin-Shaft Spiral Flow Concrete Mixer batch plant at GSL. The cement, silica fume, silica sand, and Sil co Sil were added to the batch plant, and the batch plant was programmed to add water per the mixture design. Superplasticizer was added after this, and the materials were mixed. The mixing time was not recorded. Following this, the steel fibers were added and mixed for roughly 10 minutes.

\subsubsection{Placement}

A $0.5-\mathrm{yd}^{3}$ Gar-Bro hopper on a forklift was used to transport the Cor-Tuf from the batch plant to the form. The form was located near water and steam for curing, avoiding the need to move the form while the concrete was setting. Diesel was sprayed on the form walls as a demolding agent prior to placing concrete. The total volume of the form was approximately $13.3 \mathrm{ft}^{3}\left(0.49 \mathrm{yd}^{3}\right)$. However, the hopper was not filled to its full $0.5-\mathrm{yd}^{3}$ 
capacity to avoid spillage, as the batch plant chute cannot provide the fine level of control needed. Cor-Tuf was placed from the hopper at one end of the form (Figure 3.2), and allowed to flow down the beam. This is standard procedure for beam construction. Vibration was applied to the sides of the form to aid in consolidation, but vibration was not applied to the Cor-Tuf itself, due to concerns about fiber settling and segregation.

The concrete in the first hopper load was sufficient to fill the beam to roughly $80 \%$ full. At this point, the concrete developed a "skin" as the set progressed. This skin, shown in Figure 3.3, impeded the flow of the concrete. A pallet jack was used to raise the placement end of the beam slightly to induce the concrete to flow. While the hopper was refilled, the skin was kneaded by hand to break it up and prevent the formation of a joint in the beam. Concrete from the second hopper was placed from the same location as the first hopper, but did not flow as readily. The concrete was guided by hand to fill the remainder of the beam. Due to the high fiber content, Cor-Tuf does not finish nicely. The top of the beam was given a shovel finish, which does not affect this research as the outer 2 in. on the top and bottom were avoided when taking cores. For quality control purposes, three 4 in. by 8 in. cylinders were cast at the same time as the beam.

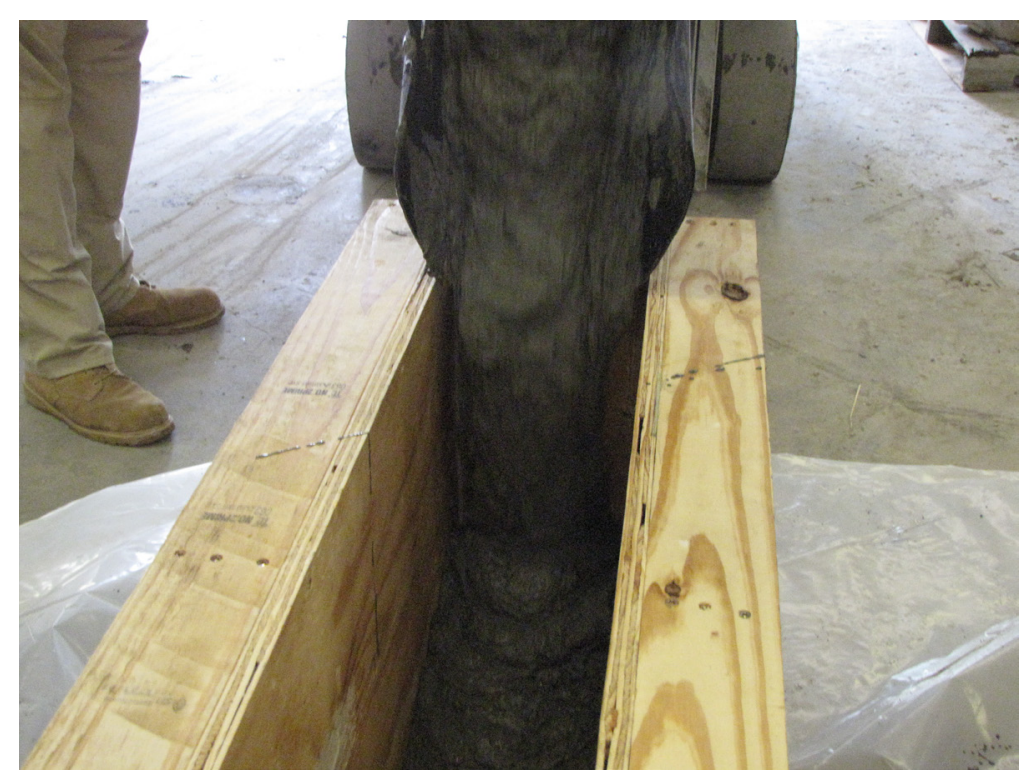

Figure 3.2. Placing Cor-Tuf at beam end. 


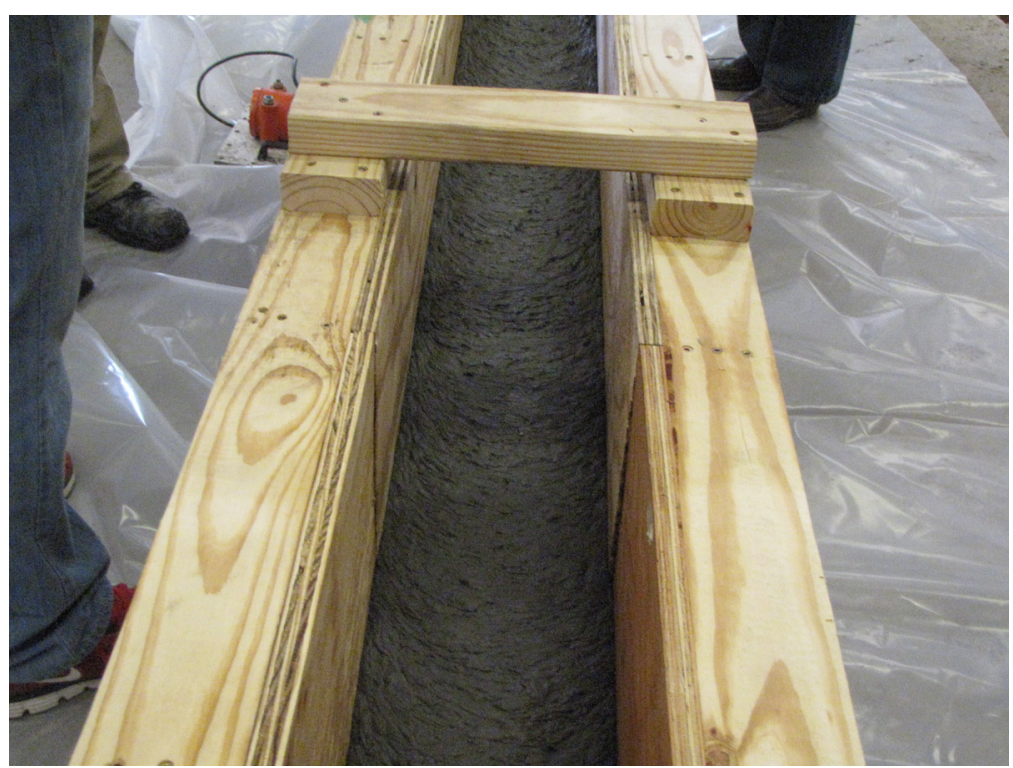

Figure 3.3. Formation of "skin" during casting.

\subsubsection{Cure regime}

The beam was covered in plastic sheeting and wetted periodically for the 24 hours following placement. Wetted burlap was placed on the beam after waiting roughly 5 hours, so that the burlap would not stick to the concrete. After the first 24 hours, a soaker hose was set up with a water timer. The burlap was inspected periodically and a timer setting was selected to keep the burlap adequately wet. A setting of 10 minutes of water every 4 hours was used. At 7 days after placement, the beam was demolded and covered with a steam blanket. The steam supply was turned off after 7 days of steam treatment (total age 14 days). To avoid rapid cooling and possible cracking, the steam blanket was vented around the edges and the beam allowed to cool slowly for another 4 days (total age 18 days) before removing the blanket. The 4 in. by 8 in. cylinders received the same cure regime, 7 days of moist curing and 7 days of steam curing in a steam room. Cylinders were then stored in ambient laboratory conditions until being tested at an age of 28 days. 


\subsubsection{Cutting}

Figure 3.4 shows a schematic of the beam, indicating the $x$-, $y$-, and $z$-directions. The $x$ axis is defined as the beam's longitudinal axis, and is positive away from the end at which the beam was cast. The $y$-axis is defined as the vertical axis, and is positive upward. Finally, the $z$-axis is defined as the through-web direction, and its positive direction is given by the right hand rule. To take cores from multiple locations in the beam, oriented in different directions, the beam needed to be cut into multiple pieces. The overall process consisted of cutting the beam into eight blocks, which were then cut into smaller prisms as needed to obtain cores. Cores were taken in each direction at various locations, which were not truly randomized (for practical reasons) but were as widely distributed as possible. Cored specimens were identified by the grid position (column number and row height) from which they came. The following sections explain this process in more detail.

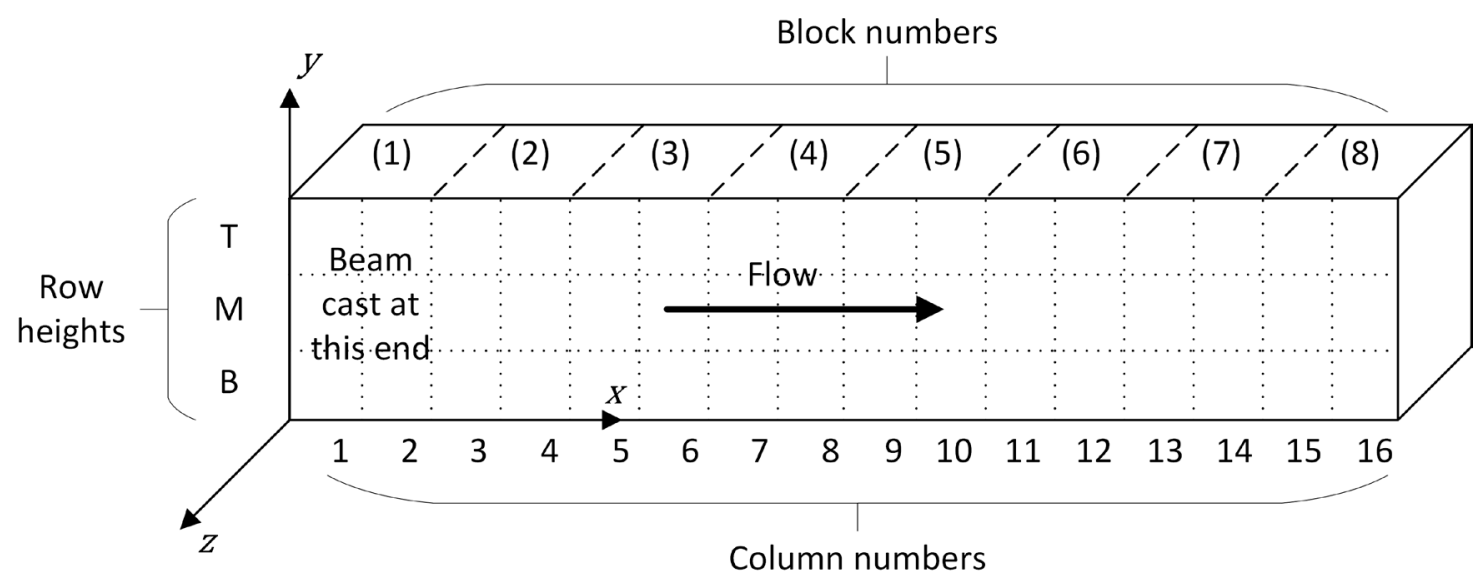

\section{Figure 3.4. Overall schematic view of beam showing grid for specimen IDs and coordinate directions.}

Because the Cor-Tuf was given a very coarse finish on the top, the beam had some mushroom-like projections on the sides, which were chipped off with a hammer. A cutting wheel was used to remove stray fibers from the edge. This was done so that the beam could be laid down flat on the table for cutting. 
Cutting was performed using a Sawing Systems (Knoxville, TN) Model 521C gantry saw, shown in Figure 3.5. The saw was mounted on a beam, and the saw fixture could move up or down as well as back and forth along the beam. The beam was supported on rails allowing for movement left or right. Before cutting, the Cor-Tuf beam was placed on the table and squared with the blade. The blade was then moved into position and advanced slowly through each cut to avoid chipping the beam. The blade was water cooled while cutting.

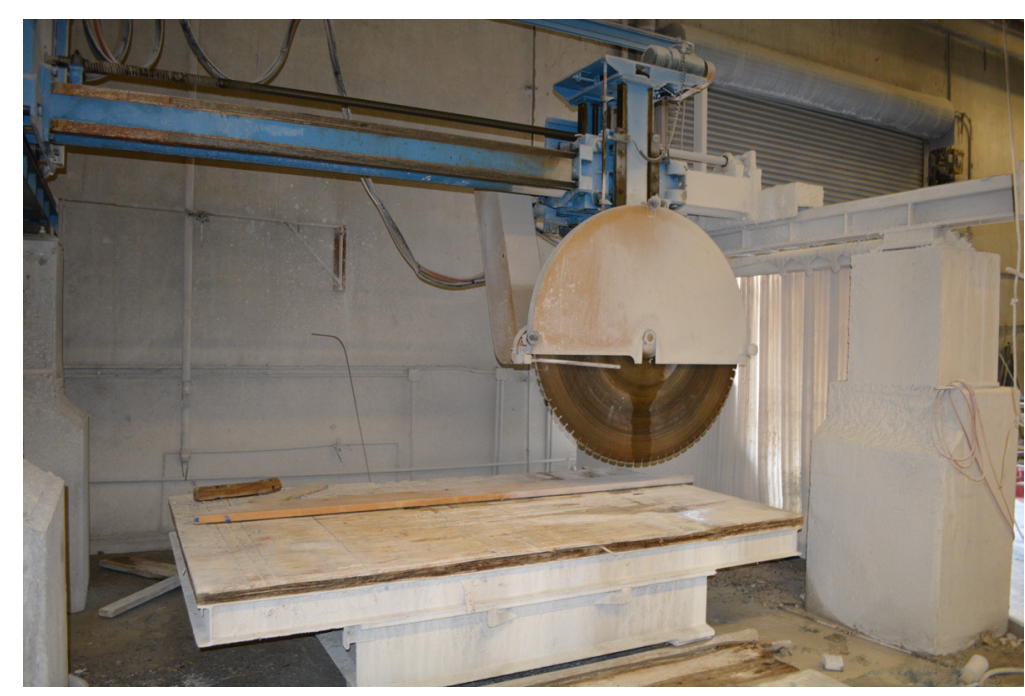

Figure 3.5. Sawing Systems Model 521C gantry saw.

First, the beam was cut into eight blocks. The six interior blocks were $14 \mathrm{in}$. wide, while the two end blocks were 18 in. wide. All blocks were 24 in. high. The top and bottom 2 in. of the beam, as well as the first 4 in. on either end, were marked and avoided when taking cores. This avoids specimens where fiber orientation would be overly influenced by form walls. For identification purposes, blocks were numbered 1 through 8, starting at the end from which the beam was cast. The age of the concrete was 19 days when blocks were cut.

Because cores in three directions were to be taken from each block, the blocks were cut into smaller prisms to facilitate coring. Figure 3.6 illustrates how the blocks were cut into prisms, with the location of the cores shown within each prism. The 
orientations of the $x$ - and $y$-axes were marked on each prism. Prisms were cut at a concrete age of $21-25$ days.

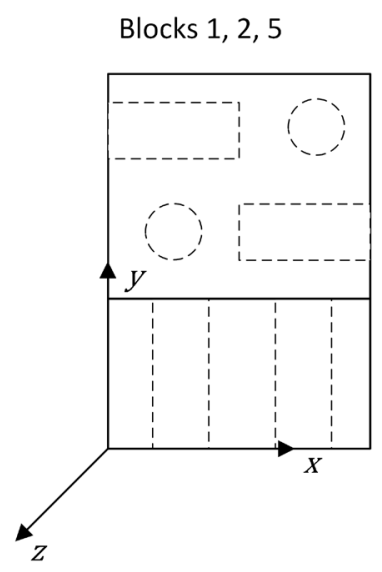

Block 3

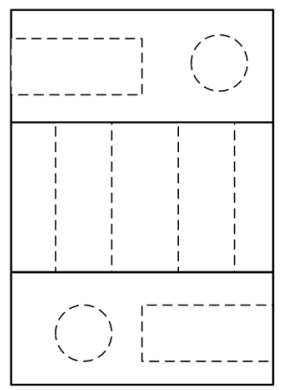

Blocks 4, 7, 8

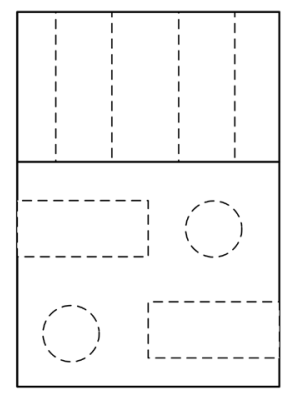

Block 6

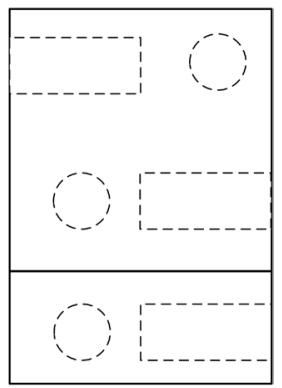

Figure 3.6. Prism cutting and coring layout.

\subsubsection{Coring}

Coring was performed using 3-in. diameter core barrels and two core drills. The drills are mounted on a steel table (Figure 3.7) so that work can be performed at a more comfortable height. Before drilling each core, a score mark was placed across the top of the core so that its original orientation could be determined. Cores were labeled with the grid position they came from, which consists of a column number ( 1 through 16$)$ and $\mathrm{T}$, $\mathrm{M}$, or B (top, middle, or bottom). Column 1 corresponds to the end of the beam from which it was cast. The $x$-, $y$-, and $z$-directions were also marked. Cores were cut to 6-in. lengths, for quasistatic test specimens, or 3-in. lengths, for dynamic test specimens. When cut, at least $1 \mathrm{in}$. was removed from the top and bottom. Cores were taken at a concrete age of 26-28 days and were stored in ambient laboratory conditions to avoid rusting the steel fibers. 


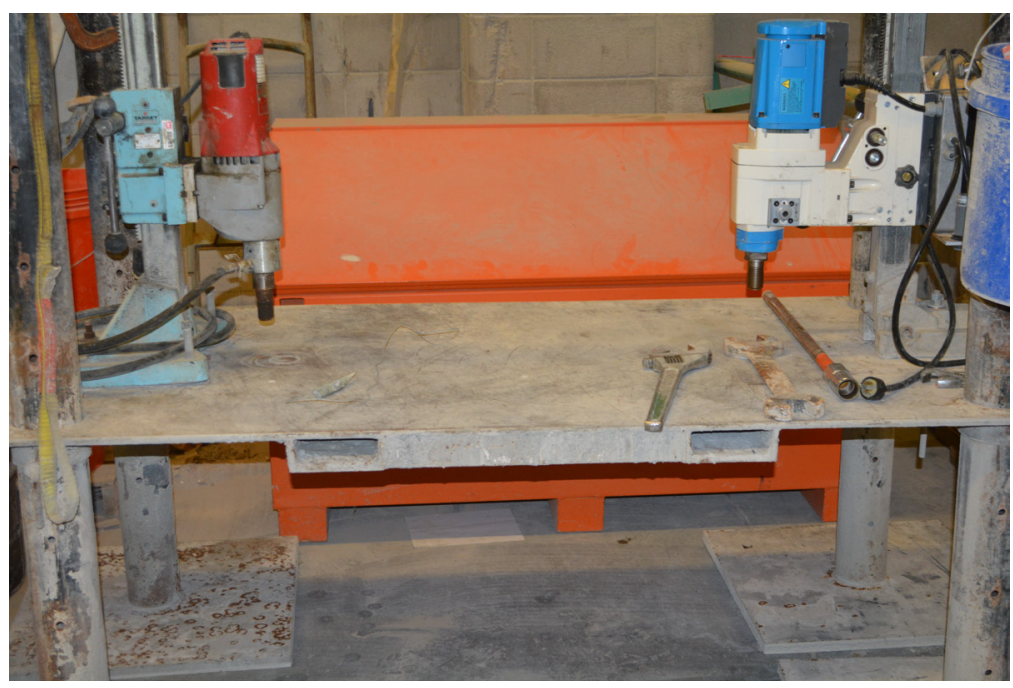

Figure 3.7. Core drills mounted to work table.

Specimen IDs incorporate the grid position, as well as information about core direction and whether the specimen is for quasistatic or dynamic testing. The ID is a string consisting of three parts, separated by hyphens, as shown in Figure 3.8. For example, X-S-7M refers to a specimen that was cored in the $x$-direction from the 7 th column at mid-height, and will be tested in quasistatic compression.

\begin{tabular}{|c|c|c|c|}
\hline AXIS & LOAD RATE & \multicolumn{2}{|c|}{ GRID POSITION } \\
\hline $\mathrm{X}$ & D - Dynamic & Column & Height \\
\hline Y & S - Quasistatic & $1-16$ & $\mathrm{~T}-\mathrm{Top}$ \\
\hline \multirow[t]{2}{*}{ Z } & & & M - Middle \\
\hline & & & B - Bottom \\
\hline
\end{tabular}

Figure 3.8. Specimen nomenclature.

\subsubsection{End grinding}

End grinding for quasistatic specimens was performed on a Marui \& Co. Ltd. Hi-Kenma model MIT-196-1-30 end grinder, shown in Figure 3.9. Some specimens required 
extensive end grinding, because of chipped ends from sawing, or because of air voids. All quasistatic specimen lengths were within $1.8 \mathrm{D}$ to $2.0 \mathrm{D}$, meeting the length tolerances from ASTM C39/C39M (ASTM 2015). Dynamic specimens received rough end grinding on the Marui Hi-Kenma end grinder, followed by fine end grinding. Fine end grinding was performed using a surface grinder to achieve a tighter tolerance than would be possible with the Marui Hi-Kenma.

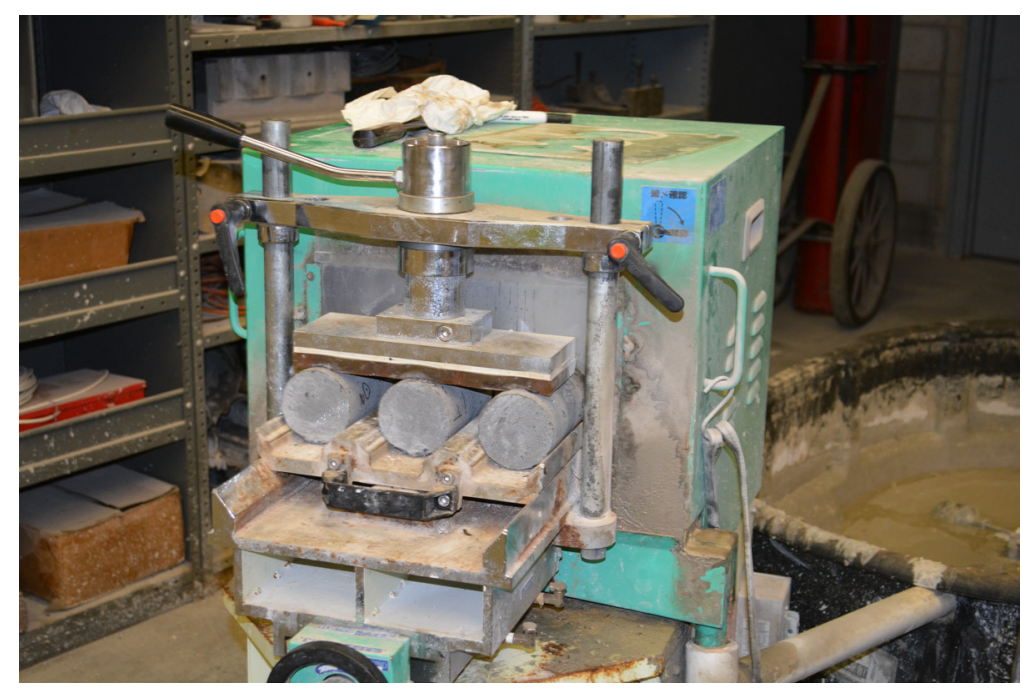

Figure 3.9. Marui Hi-Kenma end grinder.

The parallelness of the specimen ends was measured at ERDC using a dial gauge micrometer mounted on a stand, shown in Figure 3.10a. A differently-configured gauge was used at Michigan Tech (shown in Figure 3.10b). Quasistatic specimens were measured at ERDC, and dynamic specimens were measured at Michigan Tech after being shipped from ERDC. This was due to the length of time required for the fine grinder to become available. Rather than measuring total height, the micrometer was used to measure the relative height at 5 points on the cylinder ends. These were then used to calculate the deviation from parallelness. Complete measurements can be found in Appendix A. 


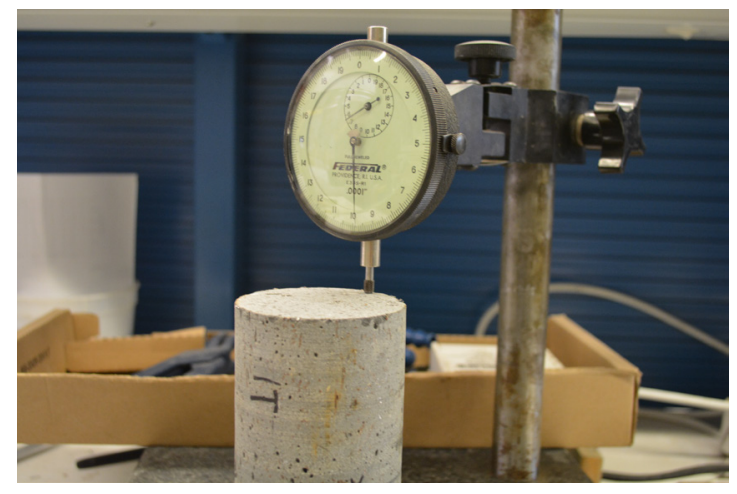

a.

Figure 3.10. Gauges for measuring end parallelness: $a$. ERDC $b$. Michigan Tech.

\subsubsection{Contrast coating}

Dynamic specimens were thinly coated with flat white enamel spray paint on one side. The coating provides a contrast for the formation of cracks, which will appear as gray on white, rather than gray on gray. Enamel, rather than acrylic, paint is used because acrylic will stretch before cracking, whereas enamel cracks with the specimen. Flat paint is selected so that the intense lights required for high speed video will not reflect off of the specimen.

\subsection{X-Ray CT}

X-ray CT scans were performed at the University of Florida's Advanced Materials Characterization Laboratory (AMCL). The AMCL has a North Star Imaging, Inc. SR450 x-ray CT system with both a $450 \mathrm{kV}$ source and a $225 \mathrm{kV}$ microfocus source. FXEControl software is used to control both sources. A movable stage is provided to hold specimens. Scans are performed using X-View CT IW software, which captures images from the detector panel and automatically rotates the stage to advance to the next radial view. The x-ray sources, specimen stage, and detector panel are all contained inside a lead-lined chamber. A closed-circuit TV system allows monitoring of operations when the door is closed. 
The NSI SR-450 has a safety system that prevents the x-ray source from being turned on unless the doors have been closed and the warning siren sounded. The warning siren is audible inside the chamber and is accompanied by flashing lights. An emergency stop button inside the chamber can be used to halt operations if needed.

All scans were completed using the $225 \mathrm{kV}$ microfocus cone-beam source. A 1/16-in. thick copper plate was placed in front of the source to filter out undesirable wavelengths, resulting in sharper images (Oesch 2015). The following paragraphs describe the steps taken to warm up, calibrate, and use the x-ray CT scanner.

\subsubsection{Startup}

Startup should be performed after powering on the x-ray source and before beginning any scans. Startup is intended to be an automated process. FXE-Control provides a "Startup" button which automatically runs through the following tasks:

Warmup - The x-ray source voltage is gradually increased until reaching the maximum voltage ( $225 \mathrm{kV}$ in this case). During this process, a lead block should be placed in front of the x-ray source to reduce wear on the detector.

Filament Adjust-Automatically selects x-ray source filament settings that "optimize image quality and extend filament service life" (Oesch 2015).

Autocenter-This step adjusts the current to the centering coils, which produce a magnetic field to slightly deflect the electron beam and ensure it is centered when impacting the target (Oesch 2015). Autocenter can either be performed as "Autocenter All," which adjusts the centering coils for the entire range of voltages that were warmed up, or as "Autocenter $\mathrm{kV}$," which adjusts the centering coils for a user-specified voltage.

FXE-Control also provides buttons for executing these tasks individually. During the experiments, it was discovered that warmup typically required several tries to successfully complete. On one occasion, warmup was attempted over 20 times before completing. This seems to have been due to a voltage overload in the high voltage generator. In some cases, a full warmup was not performed; the x-ray source was warmed up to roughly $60 \mathrm{kV}$ beyond the needed working range, and the remaining steps 
were executed manually. The full startup procedure generally took 1 hour or more. The abbreviated procedure described above required about 20-30 minutes and allowed more scans to be completed.

\subsubsection{Calibration}

Calibration should be performed after the machine has been out of use, or after altering the machine. A full calibration consists of capturing images at three different intensities: dark field, light field, and midfield. Dark field calibration is performed with the x-ray source turned off, and light field and mid-field calibrations are performed with the x-ray source turned on (Oesch 2015). No specimen is placed between the source and detector during calibration. This step could also be called a calibration check, as the resulting images are used to ensure the images contain pixels within the expected range; the images are not used for adjusting scans collected with the x-ray scanner. Calibration, or calibration checking, tests the range of x-ray intensities that the $\mathrm{x}$-ray source can produce and the detector can detect. Calibration settings based on the procedure described by Oesch (2015) are given in Table 3.2.

Table 3.2. Calibration settings (Oesch 2015).

\begin{tabular}{llll}
\hline \hline \multirow{2}{*}{ Settings } & \multicolumn{3}{c}{ Calibration step } \\
\cline { 2 - 4 } & Dark field & Light field & Midfield \\
\hline Line filter & Off & On & On \\
X-ray source & Off & On & On \\
Voltage & N/A & $180 \mathrm{kV}$ & $160 \mathrm{kV}$ \\
Current & N/A & $370 \mu \mathrm{A}$ & $270 \mu \mathrm{A}$ \\
Number of frames averaged per view & 16 & 2 & 2 \\
Target pixel intensity & 16000 & $1500-3500$ & $4000-8000$ \\
\hline \hline
\end{tabular}

The line filter setting pertains to the image acquisition software and is used for improving the quality of images. Frame averaging is also used to improve image quality by taking multiple images and averaging them before display. Note that the intensity is a 14-bit grayscale value varying from 0 , white, to $2^{14}-1=16383$, black (Yu-Min Su 2012). 


\subsubsection{Scanning}

Use of a cone beam $\mathrm{x}$-ray source requires that the specimens are elevated above the stage so that part of the beam is not blocked by the stage. Styrofoam cups were found to work well as stands for supporting the specimens: the low-density foam has an x-ray attenuation coefficient that is roughly the same as that of air. When placing the specimen on the Styrofoam, care was taken to center the cup on the stage, and the specimen on the cup. The specimen's orientation was also recorded so that the results of the scan could be correctly interpreted for fiber orientation determination.

After positioning the specimen, the x-ray was turned on (following the safety procedure). The stage was rotated and the image from the detector was inspected in XView IW CT to ensure the specimen remained in the detector's field of view during the full range of rotation. This served as an additional check on the centering of the specimen. After returning the stage to its original, zero rotation position, the scan was started. Typical settings for image acquisition control and the $\mathrm{x}$-ray source are given in Table 3.3. The x-ray source can be controlled by specifying a voltage and current, or a voltage and power. The latter option is called "Isowatt" mode, and was found to be a more intuitive way to adjust the $\mathrm{x}$-ray source.

Table 3.3. Typical range of settings for x-ray CT scanning.

\begin{tabular}{ll}
\hline \hline \multicolumn{1}{c}{ Settings } & \multicolumn{1}{c}{ Value } \\
\hline Voltage & $162-185 \mathrm{kV}$ \\
Current & $270-493.8 \mu \mathrm{A}$ \\
Power & $49.95-80 \mathrm{~W}$ \\
Number of radial views & 720 \\
Number of frames averaged per view & 1 \\
Variation limit & $2 \%$ \\
\hline \hline
\end{tabular}

Note that multiple $\mathrm{x}$-ray source settings are given. This is because these settings were varied somewhat during the course of the experiment in an attempt to obtain consistent levels of penetration between specimens. Penetration was assessed by inspecting the intensity of pixels in the center of a captured radiograph image. Ideally, 
the intensity should be at or around 1900. Settings for each scan are recorded in Appendix B. Scans typically took between 90 and 110 minutes.

The first three settings in Table 3.3 pertain to the x-ray source and are set in FXEControl. The last three settings are related to image acquisition and are set in X-View IW CT. The number of radial views is simply the number of images captured over a full rotation of the specimen. For example, 720 radial views means that images are taken at 0.5 degree rotation increments. Frame averaging can be used when the materials in the specimen are difficult to distinguish; it improves image quality by taking multiple images at each rotation step and averaging them. For Cor-Tuf, however, Oesch (2015) found that the matrix and fibers are distinct enough that frame averaging does not bring any significant benefit but only increases the required scan time. Finally, the variation limit is the maximum allowed difference between the pixel values in the current and previous images; if the variation limit is exceeded, the software will recapture the current image until the variation reduces to an acceptable level (Oesch 2015). The variation limit essentially functions to prevent the scan from continuing if a component of the scanner breaks down (Oesch 2015).

The resolution obtained in the scan is affected by the distance from the source to the specimen, and from the specimen to the detector. These distances are illustrated in Figure 3.11. The distances $D_{1}$ and $D_{2}$ are recorded with the x-ray CT scan settings in Appendix B.

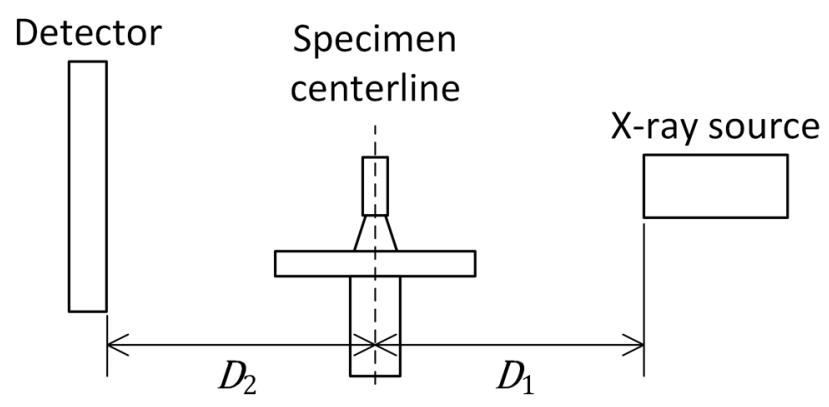

Figure 3.11. Image acquisition geometry. Not to scale. 


\subsubsection{Calibration scan}

The calibration scan is performed on a special calibration rod, which contains ball bearings embedded in plastic. The dimensions of the rod are known to the reconstruction software (North Star Imaging efx-CT), and the calibration scan is used to track the rotation of the stage during the scan. A calibration scan was performed whenever the source-to-specimen or specimen-to-detector distances were changed. Typical calibration scan settings are described below in Table 3.4; full details can be found in Appendix B.

Table 3.4. Typical settings for calibration scanning.

\begin{tabular}{ll}
\hline \multicolumn{1}{c}{ Setting } & \multicolumn{1}{c}{ Value } \\
\hline Voltage & $120 \mathrm{kV}$ \\
Current (calculated) & $683.3 \mu \mathrm{A}$ \\
Power (specified in Isowatt mode) & $82 \mathrm{~W}$ \\
Number of radial views & 60 \\
Number of frames averaged per view & 1 \\
Variation limit & $2 \%$ \\
\hline \hline
\end{tabular}

\subsubsection{Volume reconstruction}

Finally, the program efx-CT was used to reconstruct the 3-D volume data from the specimen radiographs. This process is automated, and guides the user through a number of steps in a dialog box to determine the radiographs to use, the calibration scan to use, and the region of interest (ROI) for the reconstruction, as well as the number of voxels in the reconstruction. The ROI option allows the user to exclude the air surrounding the specimen from the reconstruction process. The resolution is determined by the number of voxels and the dimensions of the ROI. Note that it is not possible to get a better resolution than the geometric setup can provide; the software simply interpolates between voxels if this is attempted. The dialog box also includes optional sections for beam hardening correction and fine $\mathrm{x}$ tuning. These were not used, as beam hardening and other artifacts were removed during the image processing described in Section 3.3. Settings for volume reconstruction can be found in Appendix B. Processing took between 4 and 6 hours depending on the number of voxels in the reconstruction. 


\subsection{Image Analysis}

The image analysis was performed in MATLAB (The MathWorks 2014b) using the methodology and code established by Oesch (2015). Modifications were made to run the most computationally-intensive portions of the code on a cluster, thus reducing the overall time needed for data processing. The data processing consisted of three general phases: pre-processing, fiber orientation analysis, and post-processing. Pre- and postprocessing were performed on a lab workstation due to the amount of data that needed to be read from and written to disk during the process. Fiber orientation analysis was processor- and memory-intensive and was performed on the Superior cluster at Michigan Tech. Although the cluster could have been used for all data processing, the time required to transfer files to and from the cluster via the network outweighed the increase in execution speed for most operations. Network transfer rates were 10-20 MB/s, depending on the network traffic.

A pilot study on the scalability of the fiber analysis code was conducted. This study provided information for deciding the most efficient way to perform data processing. Information on scalability was also a requirement for the project proposal submitted to Michigan Tech to obtain access to the Superior cluster. The pilot study was conducted on the Portage cluster, which is intended for small-scale projects and testing code. The pilot study can be found in Appendix C.

The steps in the analysis are described in the following sections. The code used is already openly available (Oesch 2015), and only slight modifications were made. Modifications were mainly to accommodate limited memory on the workstation, by only using the portions of Oesch's code that deal with quarter-scale images, or to work with the queueing system on Superior. Therefore, the code is not reproduced in this document: interested readers are encouraged to refer to Oesch (2015).

\subsubsection{Image extraction}

Images must first be converted from NSI's image format into a more convenient format for MATLAB. NSI images consist of a number of .nsidat files, with each file containing multiple slices of the image and the number of slices limited so that no file is larger than 
2 GB. For each file, the NSIExtractor function (University of Maine 2013) reads the slices, downsamples them from 32-bit floating point values to 8-bit unsigned integers, and assembles them into a MATLAB array, which is saved to disk. The downsampling process scales the floating point values to fit the range from 0 to 255 in the new data format. Scaling is controlled by selecting maximum and minimum intensities $I_{\max }$ and $I_{\min }$. Values below $I_{\min }$ are set to 0 , and values above $I_{\max }$ are set to 255 ; values in between are scaled linearly. Typically, $I_{\min }=0$ is used, corresponding to the intensity level of the voids. A value for $I_{\max }$ is selected by using ImageJ (Schneider et al. 2012) to adjust the contrast on a representative slice from the NSI image. Contrast is increased slowly to determine the lowest value of $I_{\max }$ where no significant image information is lost, i.e., no features begin to disappear.

\subsubsection{Image assembly and scaling}

After converting each file in the image to a MATLAB array, the arrays are scaled to onequarter size and assembled into a single MATLAB array for the entire image, which is then saved to disk. This is accomplished using the IAccumulatorQ function, which was adapted from the IAccumulator function (Oesch 2014d) to only assemble the quarterscale image, and not the full image. Scaling by one quarter reduces the image size 64fold, significantly reducing the required time for subsequent operations. Although scaling does result in a loss of information, this does not affect the fiber orientation calculations. The CT images have resolutions of 40-60 $\mu \mathrm{m}(0.0016-0.0024 \mathrm{in}$.), depending on the sample size, which translates to roughly 9-14 voxels across the fiber diameter. At one-quarter scale, the resolution is effectively 160-240 $\mu \mathrm{m}(0.0063-0.0094$ in.), which gives roughly 2-3 voxels across the fiber diameter. A minimum of 2 voxels across the fiber diameter is needed to determine the orientation.

\subsubsection{Shrink-wrapping}

Shrink-wrapping is the process of creating a binary array that indicates which image voxels are part of an object, in this case, the Cor-Tuf specimen. The function shrinkWrap (de Wolski 2011; modifications by Oesch 2014g) was used for this purpose. When run 
on the quarter-scale image, shrinkWrap returns a logical array with the same dimensions as the image; each entry in the logical array is true if the corresponding image voxel is within the specimen, and false otherwise.

\subsubsection{Image correction and void analysis}

The function IqtCorrect, adapted from ICorrect (Oesch 2014e) to process quarter-scale images only, corrects the image for vertical beam hardening, identifies voids, and computes some basic void properties. Vertical beam hardening is corrected by calculating the voxel intensity histogram for each slice, identifying the peak on the histogram corresponding to the Cor-Tuf matrix, and shifting the intensities of each slice so that the peak occurs at the same intensity for all slices. The intensity chosen is that of the matrix peak on the middle slice (Oesch 2015). Threshold values separating voids from matrix, and matrix from fibers, are chosen using the triangular algorithm (Young et al. 1998). Oesch (2015) found the triangular algorithm well-suited to automatic determination of threshold values for Cor-Tuf. After identifying the voids, they are rendered for visual inspection and to serve as a quick check on correct threshold determination. The surface area and volume of voids are calculated in terms of square and cubic voxels, respectively.

\subsubsection{Fiber correction and identification}

The function FCorrect (Oesch 2014b) corrects for beam hardening artifacts, which cause the fibers near the outside of the cylinder to appear thicker (Oesch 2015). Based on the resolution of the CT scan (determined during volume reconstruction, see Section 3.2.5), the width of the fiber in voxels can be determined. Using the matrix-fiber threshold determined previously, all fibers within the shrink-wrapped region are selected. The MATLAB Image Processing Toolbox (The MathWorks 2014a) connected components analysis functionality is used to identify elliptical fiber cross-sections, measure their minor axis diameter, and their distance from the specimen centroid. A quadratic polynomial fit is used to determine a relationship between diameter and distance from the centroid; this polynomial is then used to determine an intensity correction to be applied. Finally, objects with a width greater than 10 times the true fiber diameter are removed; 
this eliminates ring artifacts. Ring artifacts are simply bright circles in a reconstructed slice.

\subsubsection{Fiber orientation analysis I (cluster)}

As mentioned previously, computing the fiber orientation was computationally intensive, and was performed on the Superior cluster at Michigan Tech. The LSFfiberOrient function (Flanders 2014; modifications by Oesch 2014f) was used to determine the fiber orientation vector at each fiber voxel. This algorithm, described by Trainor et al. (2013a; 2013b), is also similar to that used by Krause et al. (2010). First, the image is smoothed by applying a Gaussian image filter. Then, the eigenvectors of the Hessian are computed at each voxel. Two of these eigenvectors correspond to the directions of most change, i.e., perpendicular to the fiber axis, moving from fiber to matrix. The third eigenvector is directed along the fiber axis, where there is the least change in image information; this eigenvector is associated with the smallest eigenvalue (Krause et al. 2010). Because the computation is performed at each voxel, independent of the other voxels, this function was determined to be a good candidate for running in parallel. Based on the scalability study (Appendix C), this function was run in parallel using 2 CPU cores.

The function FAnalysis (Oesch 2014a) was used to calculate the fiber orientation angles with the $x$-, $y$-, and $z$-axes. The angles $\theta_{x}, \theta_{y}$, and $\theta_{z}$ were calculated by solving equations $(2.1 \mathrm{a}-\mathrm{c})$. FAnalysis also performed a connected component analysis using the MATLAB Image Processing Toolbox to determine the fiber volume fraction.

\subsubsection{Fiber orientation analysis II (workstation)}

The function FStatistics (Oesch 2014c) was used to compute basic summary statistics on the fiber orientation angles. The mean, standard deviation, and pseudo-mode were calculated. The true mode is difficult to determine for continuous data such as this; the pseudo-mode is the angle corresponding to the most-populated bin in a histogram of the data. In Oesch's (2015) analysis, 180 bins were used, each spanning 0.5 degrees. The FStatistics function can also perform fiber dispersion analysis, but this feature was not used in this project. 
Finally, correctly interpreting the axes in the fiber orientation data is important. Because the specimen placement within the scanner was recorded, it was possible to determine which axes in the $\mathrm{CT}$ scan data mapped to which axes in the original beam.

\subsection{Quasistatic Compression Testing}

Currently, there is no standard for compression testing of UHPC. Therefore, quasistatic compression testing on Cor-Tuf was performed according to ASTM C39/C39M (ASTM 2015), albeit using an increased rate of loading. Research by Graybeal (2006) indicates that increasing the rate of loading from $35 \mathrm{psi} / \mathrm{s}$, as recommended in ASTM C39/39M, to $150 \mathrm{psi} / \mathrm{s}$, has a negligible effect on the compressive strength of UHPC. The UHPC loading rate of $150 \mathrm{psi} / \mathrm{s}$ was used for all quasistatic tests in this work. Tests were performed on a Baldwin 300-kip compression testing machine (model 300-CT). This machine was originally manually operated but was later upgraded by adding a controller to automate the ASTM C39/C39M test procedure. Based on the entered cross-sectional area of the specimen, the controller sets the rate of force application to conform to the stress rate in the ASTM standard. The controller does not permit a non-ASTM load rate to be entered, but the same effect can be produced by entering a cross-sectional area that is larger than that of the actual specimen. The desired rate of force application is given by

$$
\dot{P}=\dot{\sigma}_{U H P C} A_{S}=\dot{\sigma}_{U H P C} \frac{\pi}{4} D_{S}^{2}
$$

where $\dot{\sigma}_{U H P C}=150 \mathrm{psi} / \mathrm{s}$. Because the controller will only apply a stress rate of $\dot{\sigma}_{A S T M}=35 \mathrm{psi} / \mathrm{s}$, the area that should be entered is

$$
A_{\text {controller }}=\frac{\dot{\sigma}_{U H P C}}{\dot{\sigma}_{A S T M}} A_{S}=\frac{150}{35} \frac{\pi}{4} 3^{2}=30.29 \mathrm{in}^{2}
$$

The specimen length and diameter were measured before testing, using the average of three measurements. Specimen dimensions can be found in Appendix D. The end parallelness was measured after end-grinding, and is summarized in Appendix A. Before each test, specimens were placed between two steel platens and centered. Note 
that the load is applied from the bottom in this machine. The testing machine was manually advanced until the platen on top of the specimen was less than $1 / 4$ in. from the machine's upper bearing block, and centering and alignment were rechecked. Some tests were mistakenly performed with neoprene pads (unbonded caps), as discussed in Section 4.2. Later tests were performed without neoprene pads: specimens were in direct contact with the steel bearing faces of the platens. Once satisfactorily centered and aligned, specimens were tested at a stress rate of $150 \mathrm{psi} / \mathrm{s}$ until a load drop of $95 \%$ was detected (ASTM 2015). Failure modes were photographed and described using the ASTM C39/C39M fracture pattern types. Although not specifically intended for UHPC, these fracture descriptions are still a useful means of identifying different failure modes, especially in the absence of a similar classification system for UHPC. The age of all specimens at quasistatic testing was 259 days.

\subsection{Dynamic Compression Testing}

The SHPB consists of a compressed nitrogen cannon, input and output bars $12 \mathrm{ft}$ long and 3 in. in diameter, and a stop bar. A number of 3-in. diameter striker bars with different lengths are available; in this work, all tests were performed with a 12-in. long striker bar. All bars, including the striker bar, were made from $1045 \mathrm{~S}$ steel, with a tensile strength of approximately $92 \mathrm{ksi}$ (Gilbertson 2011). The SHPB is shown in Figure 3.12. Four BAM units are used to receive signals from strain gages bonded to the input and output bars. Vishay or Ellis Associates BAM-1 model units were used. Strain gages are Vishay Micro-Measurements EA-06-125AC-350 gages (Gilbertson 2011), attached at the midpoint of each bar. Two pairs of diametrically opposed gages are used for each bar, as shown in Figure 3.13. Each pair of gages is connected to a BAM-1 unit and forms one channel of output. 


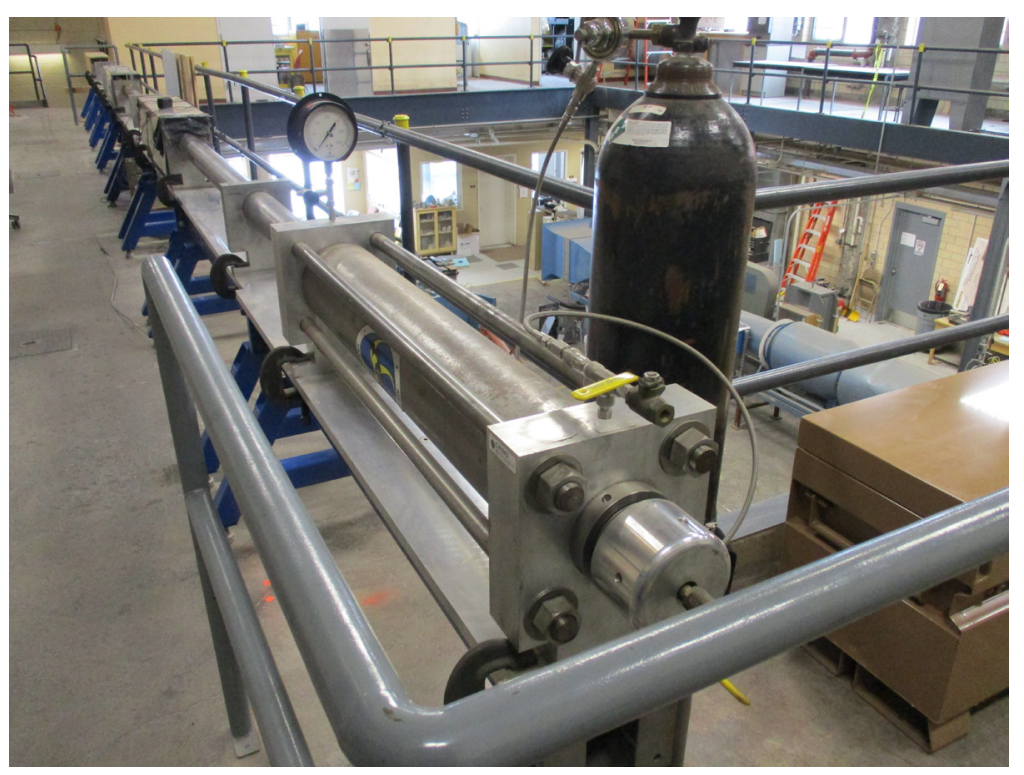

Figure 3.12. SHPB instrument looking toward output bar. The compressed nitrogen cannon is in the foreground.
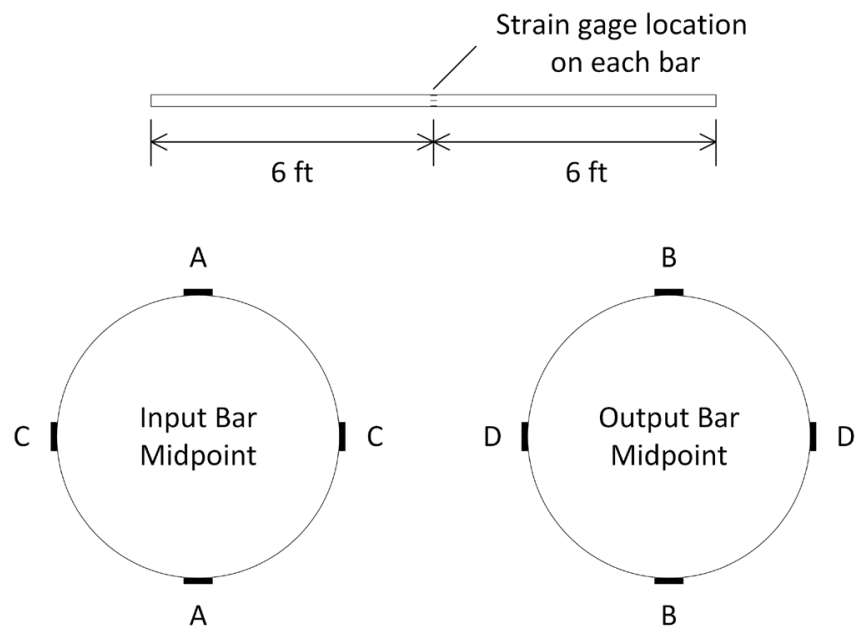

Figure 3.13. Strain gage location (after Clark 2013). Letters indicate the channel for each pair of strain gages.

The BAM-1 units were used in mode setting 4, which corresponds to a fullWheatstone bridge. However, the use of external resistors (Gilbertson 2011) results in a circuit that is more accurately described as a half-Wheatstone bridge. This setup 
compensates for bending effects introduced by nonparallelness between the bar and specimen faces, or by slight misalignment in the bearings supporting the bars (Gilbertson 2011).

The output voltages from the BAM-1 units were recorded by a Pico Technology Picoscope 4424 digital oscilloscope, which was selected based on minimum specifications determined in previous research (Gilbertson 2011). The Picoscope was controlled by software on a lab PC, which was used to save waveforms in CSV format for easy import to Microsoft Excel for data processing. Voltages were recorded using a single trigger of $400 \mathrm{mV}$, with a threshold of $10 \%$ (Gilbertson 2011). Recording starts when the voltage in any of the channels reaches $400 \mathrm{mV}$. The threshold determines the number of samples kept prior to the trigger: 2,000 samples (at roughly $1 \mu$ s increments) were recorded during the test, with 200 samples kept prior to the trigger.

The velocity of the striker bar is also recorded, as it determines the magnitude of the incident pulse. A Shooting Master Beta chronograph was used for this purpose. The chronograph has two light gates, spaced 6 in. apart, which the striker bar interrupts as it leaves the cannon. The light gates are intended to be spaced 12 in. apart, but space limitations on the SHPB prevented this. Therefore, the chronograph calculates a velocity which is twice the actual velocity of the striker. A cover is used to prevent overhead fluorescent lighting from interfering with the light gate sensors.

The following sections describe the test procedure for the SHPB. Calibration (Sections 3.5.1 through 3.5.3) should be performed once per day, before starting any tests.

\subsubsection{Bridge amplifier and meter unit calibration}

Prior to testing, the BAM-1 units were warmed up to reduce noise in the output signal. The units were then balanced to give a zero output voltage when there is no load in the bars. Finally, the BAM-1 units were calibrated to ensure consistent output levels for all units. The BAM-1 units have a calibration feature that shunts an internal resistor and unbalances the bridge, resulting in an output voltage (Clark 2013). A calibration setting of 20 was used, which should correspond to an output voltage of roughly $1.6 \mathrm{~V}$. The gain 
on each BAM-1 unit was adjusted to obtain this value when the resistor was shunted, and the actual voltage during shunting was recorded using the Picoscope. Calibration simulates a strain $\mu \varepsilon_{C A L}$ ( $\mu$ in./in.), which can be calculated as shown (Clark 2013):

$$
\mu \varepsilon_{C A L}=\frac{R_{g} \cdot C A L S E T}{G F \cdot N}=\frac{350 \cdot 20}{2.11 \cdot 1}=3317.5 \mu \mathrm{in} . / \mathrm{in} .
$$

$$
\text { where: } \quad \begin{array}{ll}
R_{g} & =\text { gage resistance }(\Omega) ; \\
C A L S E T & =\text { calibration setting (unitless); } \\
G F & =\text { gage factor (unitless); } \\
N & =\text { number of fully active gages. }
\end{array}
$$

As the filled-in terms in the equation show, the shunted resistor has a resistance of $350 \Omega$, the strain gages used have a gage factor of 2.11, and the bridge is configured with one active gage during calibration (Clark 2013).

\subsubsection{Bars together calibration}

The bars together calibration is performed with the input and output bars in contact. The procedure for testing (Section 3.5.3) is followed, with the exception that no specimen is placed between the bars. This calibration is performed as a check on proper wave transmission, and to determine a so-called stress correction factor $K_{\sigma}$. The stress correction factor is determined for the strain gages on the output bar. In one-wave analysis (see Section 2.3.2), the strain from the stress pulse transmitted into the output bar is used to determine stress in the specimen. The stress correction factor is determined as (Clark 2013)

$$
K_{\sigma}=\frac{\varepsilon_{T, \text { theoretical }}}{\varepsilon_{T, \text { measured }}}
$$

where: $\quad \varepsilon_{T, \text { theoretical }}=\quad$ theoretical strain for transmitted pulse (in./in.);

$$
\varepsilon_{T, \text { measured }}=\text { measured strain for transmitted pulse (in./in.). }
$$

The theoretical strain $\varepsilon_{T, \text { theoretical }}$ for the transmitted pulse is the same as the theoretical strain for the incident pulse given by equation (2.19). The measured strain 
$\varepsilon_{T, \text { measured }}$ is taken as the average strain over the full pulse. Because the use of a pulseshaper results in a smoothly-tapered wave, rather than a square wave, the pulse is not as well-defined. In this analysis, the full pulse is taken as an $80-\mu$ s window, approximately centered on the wave's peak.

\subsubsection{Bars apart calibration}

The bars apart calibration is performed with the input and output bars separated by approximately 2 in. As in the previous calibration, the procedure for testing (Section 3.5.3) is followed, and no specimen is placed between the bars. This calibration is performed as a check on proper wave transmission, and to determine a so-called strain correction factor $K_{\varepsilon}$. The strain correction factor is determined for the strain gages on the input bar. In one-wave analysis (see Section 2.3.2), the strain from the reflected stress pulse in the input bar is used to determine strain in the specimen. In this case, two strain correction factors are calculated, one for the incident pulse, and one for the reflected pulse. The strain correction factor for the incident pulse is determined as (Clark 2013)

$$
K_{\varepsilon, I}=\frac{\varepsilon_{I, \text { theoretical }}}{\varepsilon_{I, \text { measured }}}
$$

where: $\quad \varepsilon_{I, \text { theoretical }}=\quad$ theoretical strain for incident pulse (in./in.);

$$
\varepsilon_{I, \text { measured }}=\text { measured strain for incident pulse (in./in.). }
$$

The theoretical strain is given by equation (2.19). The measured strain is taken as the average strain over the full pulse, as described in the previous section. Equation (3.5) can also be used to calculate a strain correction factor for the reflected pulse.

\subsubsection{Specimen testing}

The age of dynamic specimens when tested was between 271 and 272 days. Specimen length and diameter were measured before testing, using the average of three measurements. The specimens were also weighed. Specimen dimensions and weights can be found in Appendix D. The following steps were performed for each test. Step 6 was skipped when performing a bars apart or bars together calibration. 
(1) The ends of all bars were wiped clean.

(2) The 12-in. striker bar was loaded into the cannon with an aluminum ramrod.

(3) The input bar was reset to its original position after any movement from a previous shot. The bar may also be moved when necessary to fit a longer striker bar into the cannon.

(4) A copper pulse shaper was attached to the end of the input bar facing the cannon. C1100 copper discs 0.75 in. in diameter and 0.085 in. thick were used, and were attached using a thin layer of AGS white lithium grease.

(5) The chronograph light box cover was placed over the space between the cannon muzzle and the input bar. The lights were turned on and checked.

(6) The end of the output bar was moved to approximately 3 in. (the specimen length) away from the end of the input bar. A thin layer of dry film lubricant (Liquid Wrench Dry Lubricant) was applied to the ends of both bars. The specimen was then placed between the bars, and the output bar was moved to provide a snug fit. A thin strip of duct tape on either end was used to hold the specimen up, preventing it from becoming dislodged before being loaded during the test.

(7) A fragment shield was placed over the specimen. Plexiglass windows on the front, top, and rear of the shield allow for video recording of tests.

(8) All data acquisition settings and triggers were checked.

(9) The cannon was filled with compressed nitrogen until a reservoir pressure of 80 psi was reached.

(10) All personnel were verified to be clear of the cannon. The cannon was fired after a countdown from three.

(11) The striker bar velocity was recorded from the chronograph readout, dividing by 2 to get the correct velocity. The waveform captured by the Picoscope was saved.

(12) The specimen and any sizable fragments were recovered and kept for later inspection. 
Note that, after completing step 9, the cannon is now live, and no one should be permitted to reach into or around the bars due to the pinching/crushing hazard that exists.

\subsubsection{High speed video}

High speed video recording of the dynamic compression tests was performed by a technician from the U.S. Army Corps of Engineers. A Phantom 7.1 camera was used, and recording was initiated by an acoustic trigger. Video was captured at a frame size of $256 \times 256$ pixels covering an area of roughly 4 in. by $4 \mathrm{in}$. The frame rate was approximately $26,000 \mathrm{fps}$ with a $2-\mu \mathrm{s}$ exposure for each frame.

\subsubsection{Data processing}

The Excel spreadsheet program developed by Clark (2013) was used with slight modification. In addition to the extensive calibration calculations and one-wave analysis in the existing spreadsheet, calculations for stress (equation (2.14)), strain (equation (2.11)), and strain rate (equation (2.12)) using the three-wave equations were added. Strain was determined by integrating strain rate using the rectangular rule with a time step of $1 \mu \mathrm{s}$. A calculation for the degree of stress equilibrium was also added, following equation (2.21). 



\section{CHAPTER 4 RESULTS}

\subsection{Fiber Orientation}

Due to the limited scan time available, all specimens except X-S-7M, Y-S-9B, and Z-S$12 \mathrm{~T}$ were scanned. The quasistatic specimens that were left unscanned were selected on the basis of having large voids or areas of incomplete hydration, which might affect compressive strength and mask fiber orientation effects. Therefore, a total of 33 specimens were scanned, 18 dynamic specimens and 15 quasistatic specimens. Unfortunately, the images from specimens Y-D-4B and Z-D-5B were filled with a number of bubble artifacts, rendering the data useless for image processing. The number of usable scans was then 31. Specimens Y-D-4B and Z-D-5B were the first specimens scanned on the first and second days of scanning in February, respectively. The artifacts could have been caused by the x-ray scanner not being fully warmed up despite running through the startup procedure. Figure 4.1 compares a slice containing bubble artifacts with a slice from a typical specimen.

$a$.

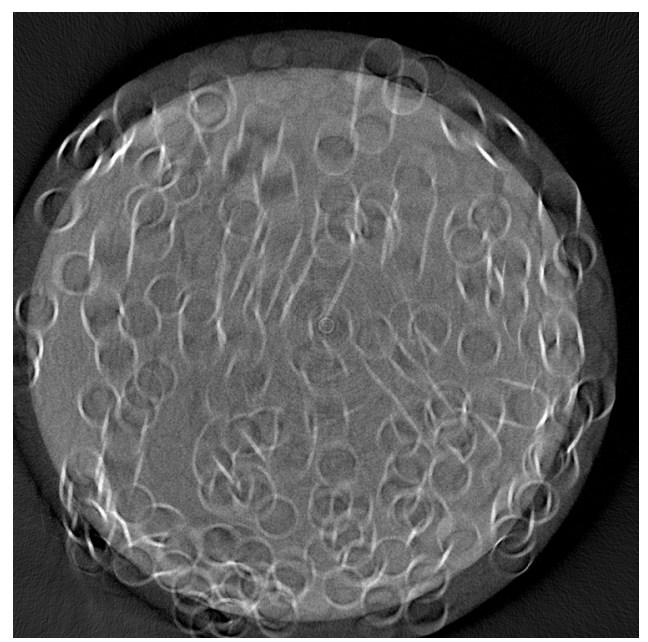

$b$.

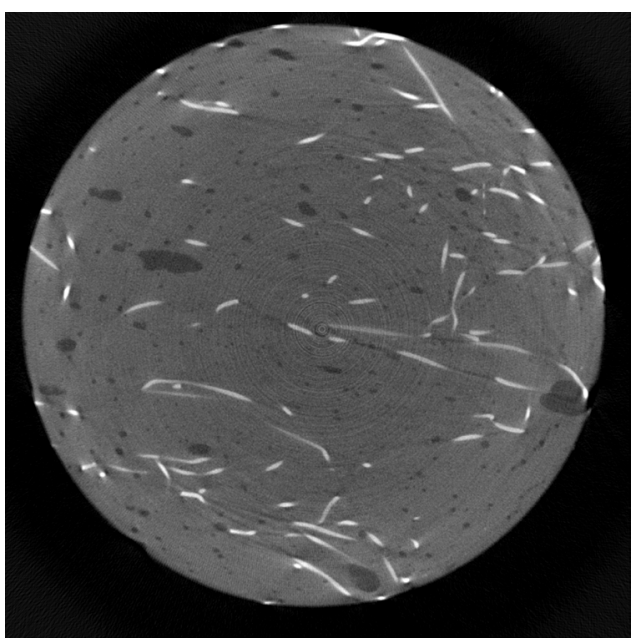

Figure 4.1. CT scan slices $a$. bubble artifacts in specimen Y-D-4B (slice 1000 of 2201) b. typical cross-section from specimen Y-D-2B (slice 1000 of 1996). 
Figure 4.2 is a rendering of only the fibers in specimen X-D-2M. This image illustrates that the fiber orientation varies throughout the specimen, and also shows that fibers are not evenly dispersed. Fiber dispersion is not considered in this research, however. It is difficult to discern many details in the picture due to the high fiber content of Cor-Tuf. The green lines indicate the vertices of the region of interest, which was set during volume reconstruction (Section 3.2.5).

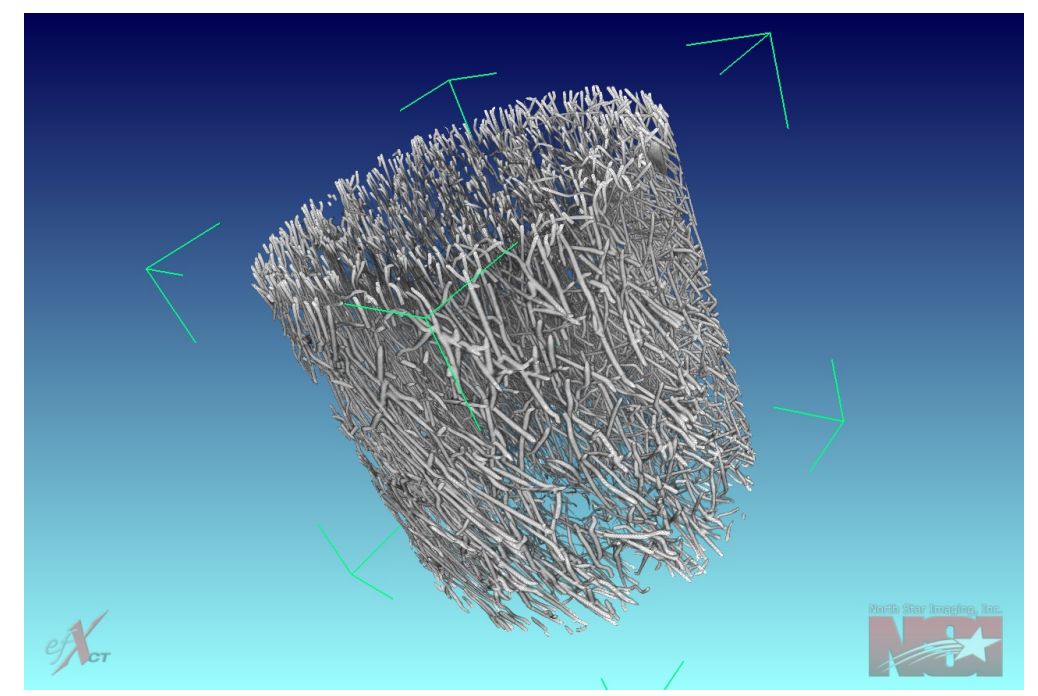

Figure 4.2. Rendering of fibers in specimen X-D-2M. Image courtesy of Charlie Burchfield. Reproduced with permission. 


\subsubsection{Specimen-level}

The specimen orientation angles, calculated using image processing methods, are summarized in Table 4.1. For the orientation angle with each axis $(x, y$, and $z)$, the mean and standard deviation of all the voxel orientation vectors is given. The pseudo-mode (PM) angle is also given (see Section 3.3.7 for definition of PM). These results will be discussed in Section 5.1 and compared to what would be obtained from randomlyoriented fibers. Note that COV is not a meaningful statistic when applied to angles, as the angles obtained depend on the reference from which they are measured. Suppose that one specimen has a mean orientation angle of 1 degree and a standard deviation of 10 degrees, while a second specimen has a mean orientation angle of 50 degrees and a standard deviation of 10 degrees. The scatter is the same for both specimens, but the $\mathrm{COV}$ would indicate that it is far greater for the first specimen. Also, if the orientation angle were measured from a different axis, the mean would change. This would change the COV, even though the scatter would not have changed.

The CT scan data were also used to determine volume fractions of the components (matrix, fibers, and voids) in each sample. These are given in Table 4.2. The volume fraction of cementitious matrix was fairly consistent, with a mean of 0.940 and a COV of $0.8 \%$. The volume fraction of voids, or porosity, was more variable, with a mean of 0.031 and a COV of $12.9 \%$. The mixture design used a nominal fiber volume fraction of 0.0315 (3.15 vol\%). However, the measured fiber volume fraction ranged from 0.021 to 0.040 , with a mean of 0.030 and a COV of $21.4 \%$. This indicates that the fiber content varies significantly throughout the beam. 
Table 4.1. Fiber orientation angles (degrees) calculated from CT scan data.

\begin{tabular}{|c|c|c|c|c|c|c|c|c|c|}
\hline \multirow{2}{*}{$\begin{array}{l}\text { Specimen } \\
\text { ID }\end{array}$} & \multicolumn{3}{|c|}{ Beam $x$-direction } & \multicolumn{3}{|c|}{ Beam $y$-direction } & \multicolumn{3}{|c|}{ Beam z-direction } \\
\hline & Mean & PM* & St Dev & Mean & $\mathbf{P M}$ & St Dev & Mean & $\mathbf{P M}$ & St Dev \\
\hline $\mathrm{X}-\mathrm{D}-2 \mathrm{M}$ & 42.1 & 25.5 & 22.9 & 73.2 & 87.0 & 14.4 & 57.5 & 89.5 & 20.8 \\
\hline Y-D-2B & 33.1 & 17.5 & 19.9 & 75.7 & 83.0 & 11.4 & 64.5 & 78.5 & 18.9 \\
\hline X-D-3T & 34.8 & 16.5 & 21.8 & 74.0 & 89.5 & 13.9 & 64.5 & 89.5 & 19.6 \\
\hline Z-D-3M & 45.2 & 32.5 & 22.1 & 70.8 & 81.0 & 14.9 & 56.1 & 61.5 & 21.0 \\
\hline Y-S-3B & 32.5 & 17.0 & 20.4 & 75.1 & 82.5 & 11.4 & 65.8 & 88.5 & 19.4 \\
\hline Z-D-4T & 33.3 & 16.5 & 21.4 & 74.0 & 88.0 & 14.4 & 66.3 & 82.5 & 19.0 \\
\hline $\mathrm{X}-\mathrm{S}-5 \mathrm{~T}$ & 35.2 & 24.5 & 20.1 & 72.6 & 79.0 & 13.2 & 65.2 & 90.0 & 19.0 \\
\hline Y-D-5M & 51.2 & 41.0 & 19.2 & 65.7 & 66.5 & 15.0 & 54.2 & 61.5 & 21.4 \\
\hline Y-S-6M & 52.1 & 44.5 & 16.9 & 60.6 & 54.0 & 14.0 & 57.4 & 70.0 & 21.1 \\
\hline X-D-6B & 32.6 & 17.5 & 21.1 & 74.4 & 90.0 & 13.8 & 66.6 & 90.0 & 18.6 \\
\hline Y-D-7T & 31.5 & 18.0 & 20.0 & 76.4 & 83.5 & 10.8 & 65.8 & 89.5 & 19.3 \\
\hline Z-S-7B & 32.2 & 18.0 & 19.7 & 74.0 & 90.0 & 13.3 & 67.0 & 81.0 & 17.3 \\
\hline Y-S-8T & 39.2 & 35.5 & 19.9 & 71.1 & 81.0 & 12.7 & 61.6 & 89.5 & 19.8 \\
\hline Z-S-8M & 44.4 & 33.0 & 21.0 & 68.6 & 79.5 & 15.7 & 58.8 & 75.5 & 20.2 \\
\hline X-S-8B & 33.2 & 20.0 & 19.8 & 73.7 & 85.5 & 13.2 & 66.3 & 89.0 & 18.0 \\
\hline X-D-9T & 33.8 & 16.0 & 22.1 & 73.0 & 89.0 & 14.7 & 66.4 & 88.0 & 18.0 \\
\hline Z-D-9M & 43.7 & 30.0 & 20.8 & 67.6 & 69.0 & 15.2 & 60.4 & 76.0 & 20.3 \\
\hline Z-D-10T & 35.3 & 18.0 & 21.3 & 73.1 & 88.0 & 14.3 & 64.4 & 79.5 & 18.0 \\
\hline X-S-10M & 52.8 & 41.5 & 16.8 & 62.2 & 57.0 & 15.2 & 55.4 & 52.0 & 21.0 \\
\hline Y-D-10B & 39.0 & 28.0 & 19.4 & 69.9 & 73.0 & 12.1 & 63.1 & 88.0 & 20.0 \\
\hline X-D-11T & 34.4 & 16.5 & 22.0 & 74.7 & 85.0 & 13.6 & 64.5 & 89.0 & 19.9 \\
\hline Z-S-11M & 41.5 & 31.0 & 21.4 & 69.0 & 89.5 & 16.6 & 61.8 & 73.0 & 18.9 \\
\hline Z-D-11B & 35.4 & 26.5 & 19.0 & 69.1 & 75.5 & 14.7 & 67.9 & 79.5 & 16.9 \\
\hline X-D-12M & 46.4 & 36.0 & 20.2 & 66.8 & 67.0 & 15.0 & 58.2 & 72.0 & 20.6 \\
\hline X-S-12B & 31.8 & 19.0 & 19.3 & 74.1 & 88.5 & 13.0 & 67.5 & 90.0 & 17.3 \\
\hline Y-D-13T & 34.5 & 20.0 & 20.4 & 75.3 & 81.0 & 11.0 & 63.3 & 85.5 & 19.9 \\
\hline $\mathrm{X}-\mathrm{S}-13 \mathrm{M}$ & 46.8 & 38.0 & 19.1 & 67.8 & 68.5 & 14.0 & 56.8 & 70.5 & 20.5 \\
\hline Z-S-13B & 36.3 & 23.5 & 20.0 & 70.2 & 90.0 & 15.9 & 65.9 & 75.5 & 16.5 \\
\hline Y-S-14T & 43.8 & 37.5 & 20.3 & 71.1 & 75.0 & 11.8 & 56.7 & 64.5 & 21.1 \\
\hline Z-S-14M & 40.5 & 29.5 & 21.4 & 70.3 & 85.5 & 15.8 & 61.6 & 76.5 & 19.4 \\
\hline Y-S-15T & 42.1 & 30.0 & 21.3 & 73.7 & 81.0 & 11.2 & 56.6 & 90.0 & 21.9 \\
\hline Mean of means & 39.0 & & & 71.2 & & & 62.2 & & \\
\hline St Dev of means & 6.4 & & & 3.9 & & & 4.2 & & \\
\hline
\end{tabular}

*PM = Pseudo-mode angle (see Section 3.3.7 for definition). 
Table 4.2. Specimen composition by volume from CT scan data.

\begin{tabular}{llll}
\hline \hline & \multicolumn{3}{c}{ Volume fraction $(-)$} \\
\cline { 2 - 4 } Specimen ID & Matrix & Voids & Fibers \\
\hline X-D-2M & 0.936 & 0.028 & 0.036 \\
Y-D-2B & 0.955 & 0.023 & 0.022 \\
X-D-3T & 0.939 & 0.020 & 0.040 \\
Z-D-3M & 0.945 & 0.027 & 0.028 \\
Y-S-3B & 0.954 & 0.025 & 0.021 \\
Z-D-4T & 0.944 & 0.027 & 0.029 \\
X-S-5T & 0.928 & 0.034 & 0.039 \\
Y-D-5M & 0.942 & 0.027 & 0.031 \\
Y-S-6M & 0.941 & 0.030 & 0.029 \\
X-D-6B & 0.930 & 0.033 & 0.037 \\
Y-D-7T & 0.941 & 0.037 & 0.022 \\
Z-S-7B & 0.946 & 0.028 & 0.025 \\
Y-S-8T & 0.948 & 0.031 & 0.021 \\
Z-S-8M & 0.945 & 0.028 & 0.027 \\
X-S-8B & 0.927 & 0.034 & 0.039 \\
X-D-9T & 0.929 & 0.033 & 0.038 \\
Z-D-9M & 0.933 & 0.038 & 0.029 \\
Z-D-10T & 0.941 & 0.033 & 0.027 \\
X-S-10M & 0.936 & 0.032 & 0.032 \\
Y-D-10B & 0.942 & 0.032 & 0.026 \\
X-D-11T & 0.924 & 0.037 & 0.039 \\
Z-S-11M & 0.945 & 0.030 & 0.025 \\
Z-D-11B & 0.944 & 0.029 & 0.027 \\
X-D-12M & 0.935 & 0.031 & 0.035 \\
X-S-12B & 0.927 & 0.035 & 0.039 \\
Y-D-13T & 0.942 & 0.034 & 0.024 \\
X-S-13M & 0.937 & 0.030 & 0.033 \\
Z-S-13B & 0.943 & 0.032 & 0.025 \\
Y-S-14T & 0.945 & 0.032 & 0.024 \\
Z-S-14M & 0.943 & 0.031 & 0.026 \\
Y-S-15T & 0.950 & 0.029 & 0.021 \\
\hline \hline & 0.940 & 0.031 & 0.030 \\
COV & $0.8 \%$ & $12.9 \%$ & $21.4 \%$ \\
\hline
\end{tabular}




\subsubsection{Beam-level}

Fiber orientation in the beam was thought to be predominantly affected by alignment in the direction of flow. The following figures illustrate the variation of fiber orientation as a function of distance from the end at which the beam was cast. The distance is measured to the center of the region (roughly $6 \times 6 \times 8$ in.) from which each core was taken. Recall the axes defined in Figure 3.4: the $x$-axis is along the length of the beam, the $y$-axis is vertical, and the $z$-axis goes across the web thickness. Figure 4.3 displays results for fiber orientation angle relative to the $x$-axis, Figure 4.4 displays results for fiber orientation angle relative to the $y$-axis, and Figure 4.5 displays results for fiber orientation angle relative to the $z$-axis. Each figure is broken into three subfigures, for the top, middle, and bottom thirds of the beam. Heights are classified in this way owing to the varying layout necessary to get cores in different directions.

Note that the $y$-direction and $z$-direction orientation angles are much higher than the orientation angles in the $x$-direction. A low orientation angle means that a fiber is more closely aligned with that axis, so this is consistent with fibers aligning in the direction of flow, which is along the $x$-axis. Consider a perfectly aligned fiber, which is parallel to the $x$-axis and has an $x$-axis orientation angle of 0 degrees. The fiber will then be perpendicular to the $y$ - and $z$-axes (orientation angle of 90 degrees). In the beam, fibers did not perfectly align, but the qualitative trend toward high $y$ - and $z$-direction orientation angles is still seen. Fiber orientation will be further analyzed in Chapter 5 .

Results also suggest that the mean angles for specimens from the middle third of the beam are different from the other two heights. For the $x$-direction (Figure 4.3), the mean angles at mid-height are greater than those at either the top or the bottom. Hence, the fibers at mid-height appear to be less aligned with the $x$-axis. At the bottom of the beam, wall effects from the formwork may play a role. There is no wall at the top, though finishing may have influenced some portion of the concrete at the top. For the $y$ and $z$-directions (Figure 4.4 and Figure 4.5, respectively), the mean angles at mid-height are less than those at either the top or the bottom. Using the same reasoning as in the previous paragraph, this is consistent with fibers at mid-height being less aligned in the $x$ direction. 


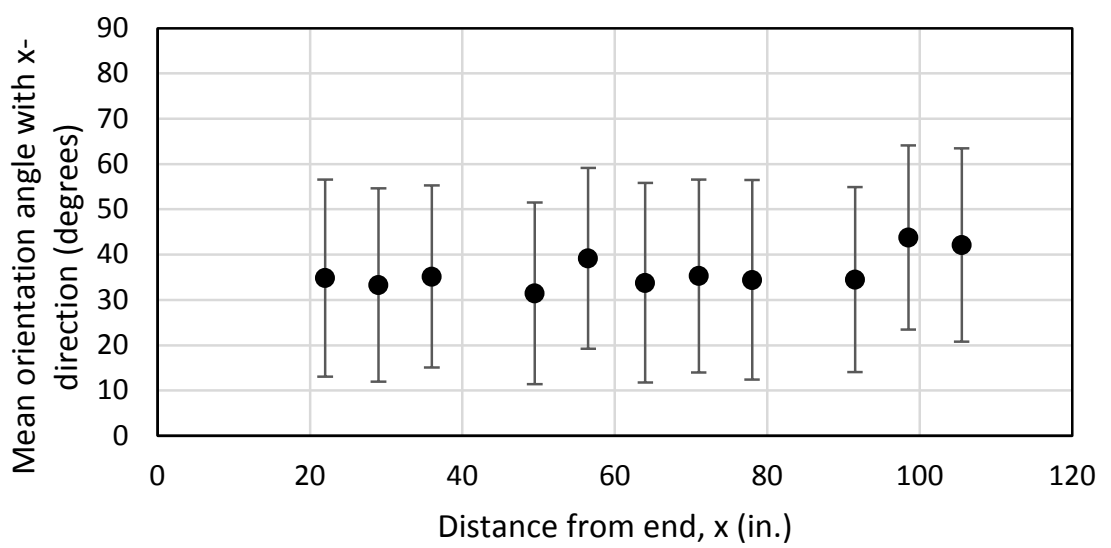

$a$.

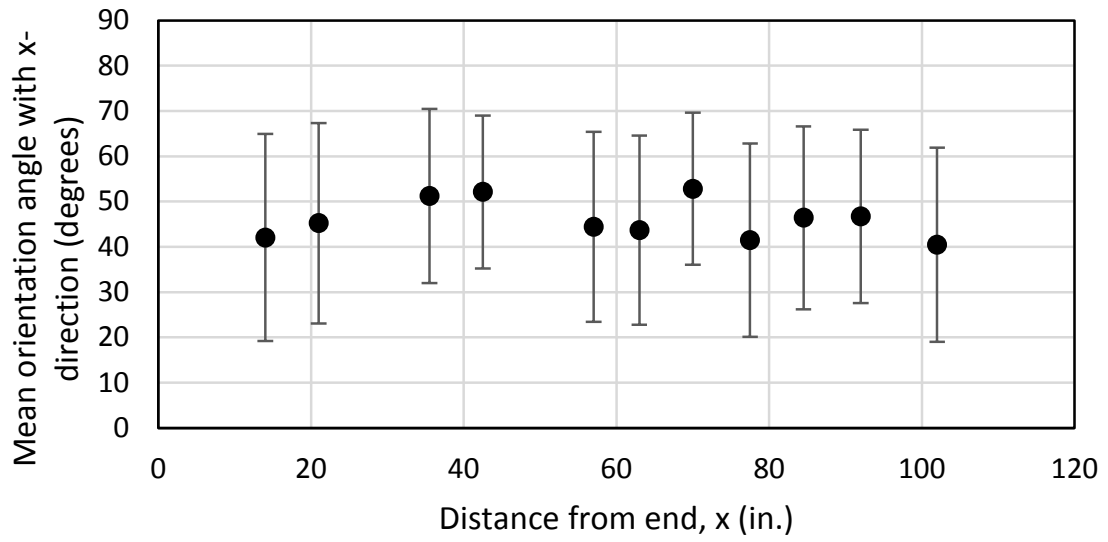

$b$.

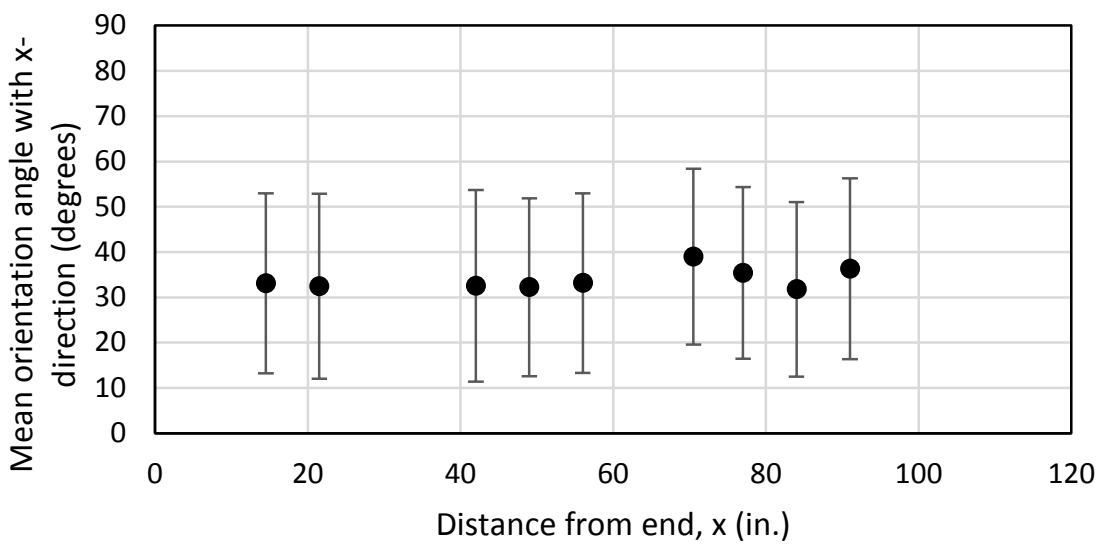

$c$.

Figure 4.3. Variation of mean $x$-direction orientation angle with distance from casting end of beam, at three heights in the beam: $a$. top, $b$. middle, and $c$. bottom. Error bars show \pm 1 standard deviation. 


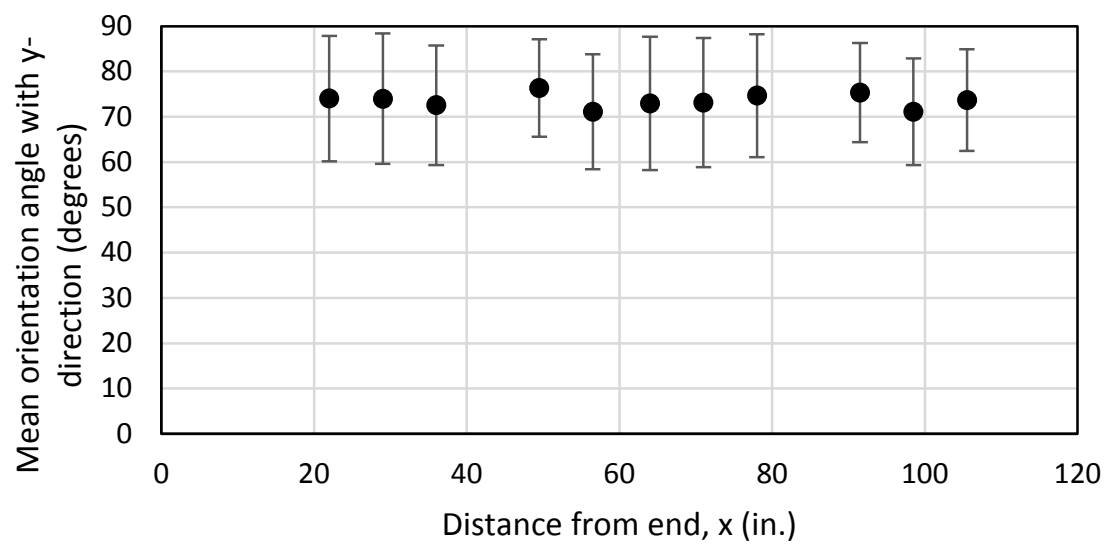

a.

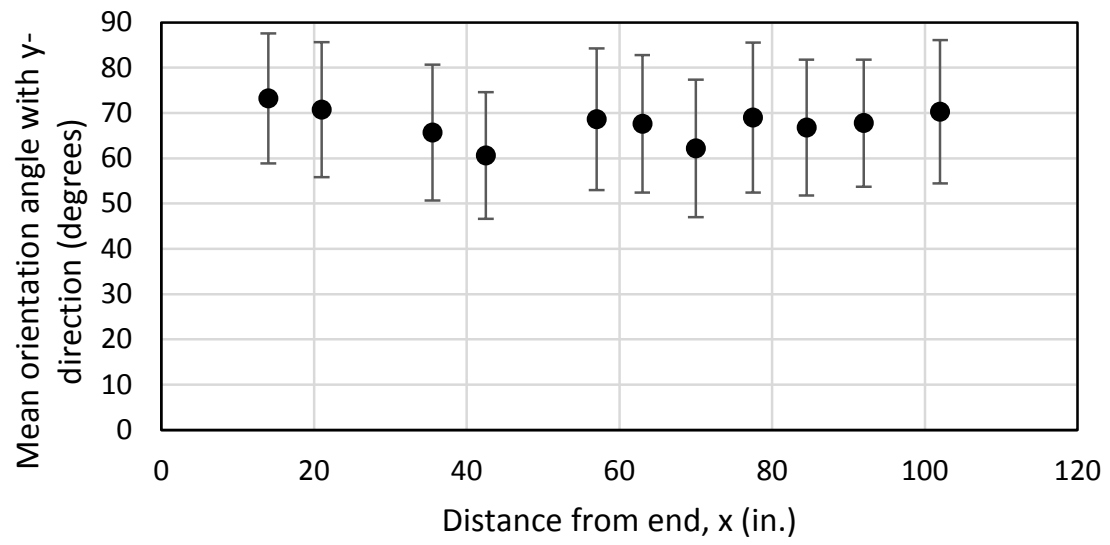

$b$.

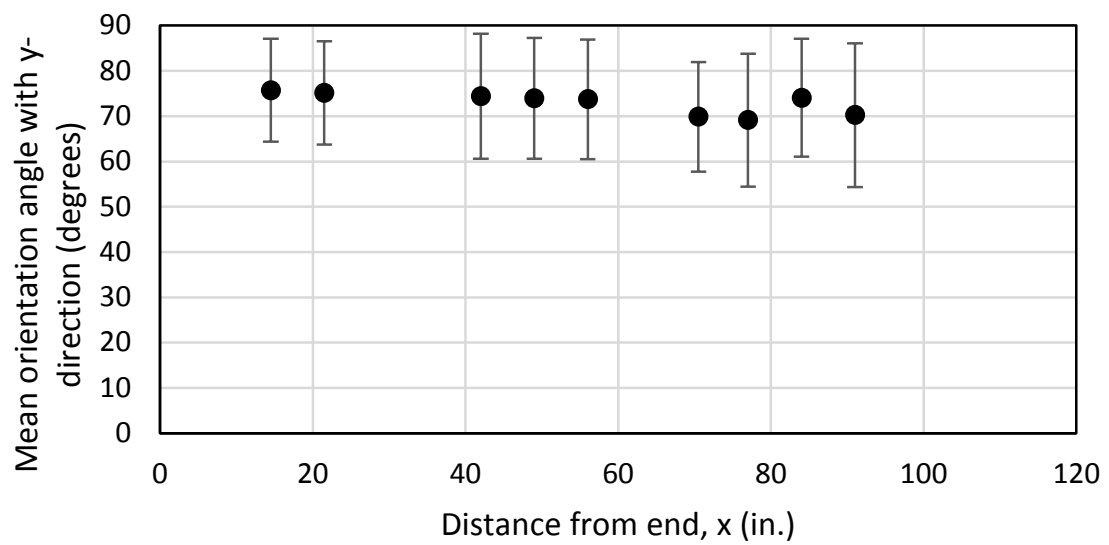

$c$.

Figure 4.4. Variation of mean $y$-direction orientation angle with distance from casting end of beam, at three heights in the beam: $a$. top, $b$. middle, and $c$. bottom. Error bars show \pm 1 standard deviation. 


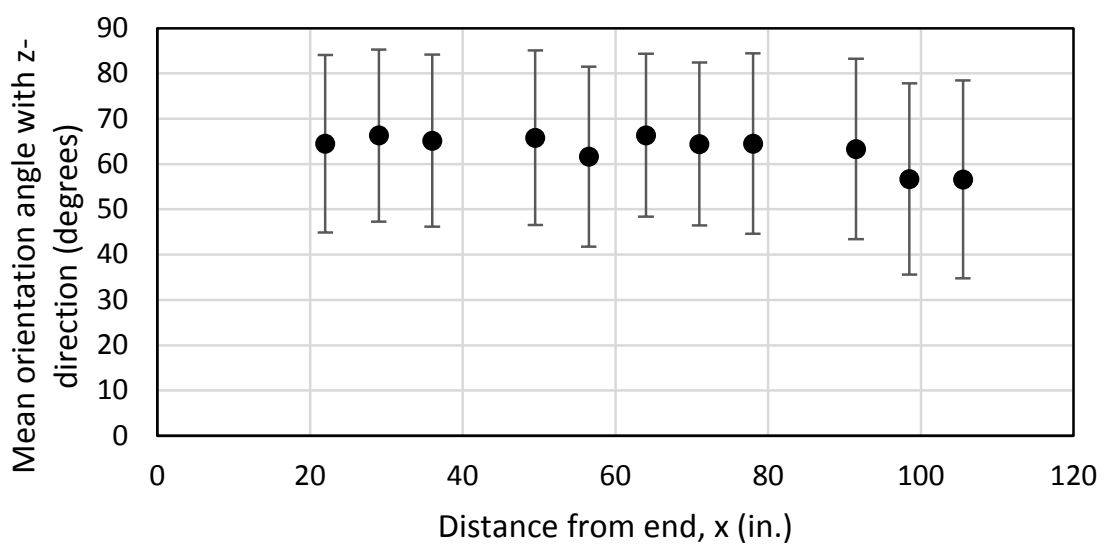

$a$.

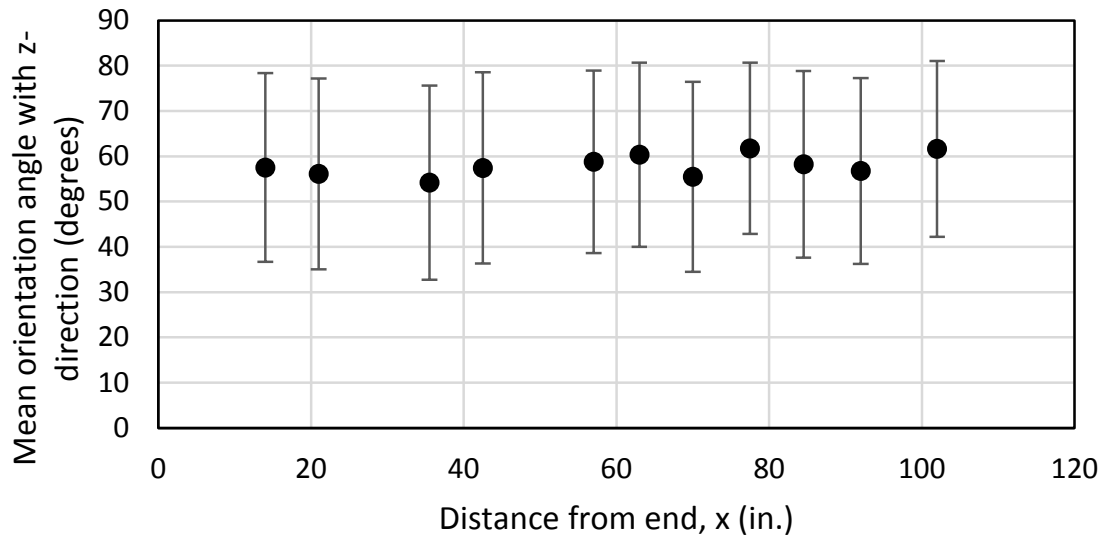

$b$.

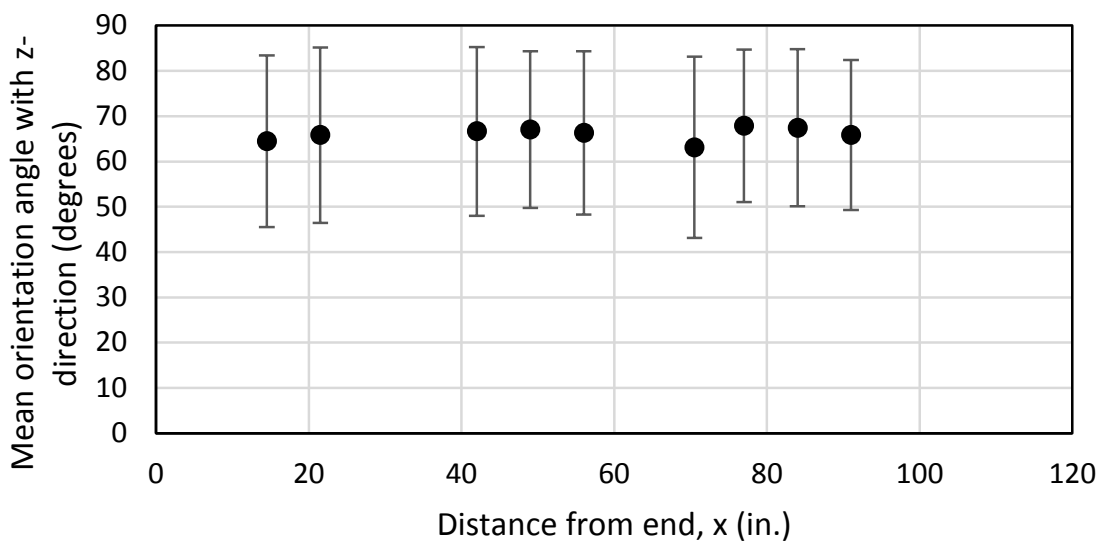

$c$.

Figure 4.5. Variation of mean $z$-direction orientation angle with distance from casting end of beam, at three heights in the beam: $a$. top, $b$. middle, and $c$. bottom. Error bars show \pm 1 standard deviation. 


\subsection{Quasistatic Compression Testing}

The results of quasistatic compression testing are summarized in Table 4.3. Columnar failures predominated. The highest strength obtained was $23.61 \mathrm{ksi}$, whereas the majority of specimens had strengths in the 15-17 ksi range and some approached $14 \mathrm{ksi}$. This is far lower than the published value of $34 \mathrm{ksi}$ (Williams et al. 2009). The use of incorrect end conditions (neoprene pads with steel retaining ring) was eventually determined to be the cause. Therefore, additional tests were conducted without the pads. The additional tests are described later in this section.

Table 4.3. Quasistatic compressive strength of Cor-Tuf specimens*.

\begin{tabular}{lcccl}
\hline \multirow{2}{*}{$\begin{array}{c}\text { Specimen } \\
\text { ID }\end{array}$} & \multirow{2}{*}{ Peak load } & Compressive & \multicolumn{2}{l}{ Failure mode } \\
\cline { 5 - 5 } & strength, & $\boldsymbol{f}_{\text {cs }}$ (ksi) & Type & \multicolumn{1}{c}{ Remarks } \\
\hline Y-S-3B & 137,776 & 19.33 & 5 & Side fracture \\
X-S-5T & 118,717 & 16.61 & 3 & Columnar \\
Y-S-6M & 153,864 & 21.44 & 6 & Side fractures, pointed end \\
X-S-7M & 105,617 & 14.71 & 3 & Columnar \\
Z-S-7B & 132,119 & 18.56 & 3 & Columnar \\
Y-S-8T & 123,647 & 17.27 & 3 & Columnar \\
Z-S-8M & 114,260 & 16.05 & 3 & Columnar \\
X-S-8B & 124,032 & 17.31 & 2 & Cone-and-split \\
Y-S-9B & 113,606 & 15.81 & 3 & Columnar \\
X-S-10M & 109,379 & 15.24 & 5 & Side fracture \\
Z-S-11M & 120,125 & 16.56 & 3 & Columnar \\
Z-S-12T & 101,855 & 14.28 & 3 & Columnar \\
X-S-12B & 121,968 & 17.00 & 3 & Columnar \\
X-S-13M & 109,053 & 15.21 & 3 & Columnar \\
Z-S-13B & 114,817 & 16.06 & 3 & Columnar \\
Y-S-14T & 148,411 & 20.77 & $4 / 5$ & Shear with side fracture \\
Z-S-14M & 126,515 & 17.70 & 3 & Columnar \\
Y-S-15T & 169,104 & 23.61 & 5 & Side fracture \\
\hline & Mean & 17.42 & & \\
& St Dev & 2.49 & & \\
\hline \hline
\end{tabular}

\footnotetext{
${ }^{*}$ Cored specimens tested with neoprene pads, age $=259$ days.
} 
Out of a total of 18 specimens, 12 failed by columnar fracture, 5 failed by side fracture [either type 5 or 6 (ASTM 2015)], and 1 failed by cone-and-split fracture. Figure 4.6 illustrates columnar and side fracture failures. Note that both specimens shown have unhydrated areas, which appear as white or beige against the gray matrix. These unhydrated, or sometimes incompletely hydrated, regions appeared in several specimens but did not seem to initiate failure. It is likely that the low w/c is responsible for the incomplete hydration. For columnar failures, cracks formed vertically, parallel to the applied load. It is expected that fibers perpendicular to the load, and therefore perpendicular to the cracks, will be most effective in crack bridging. This will be analyzed in Section 5.3.
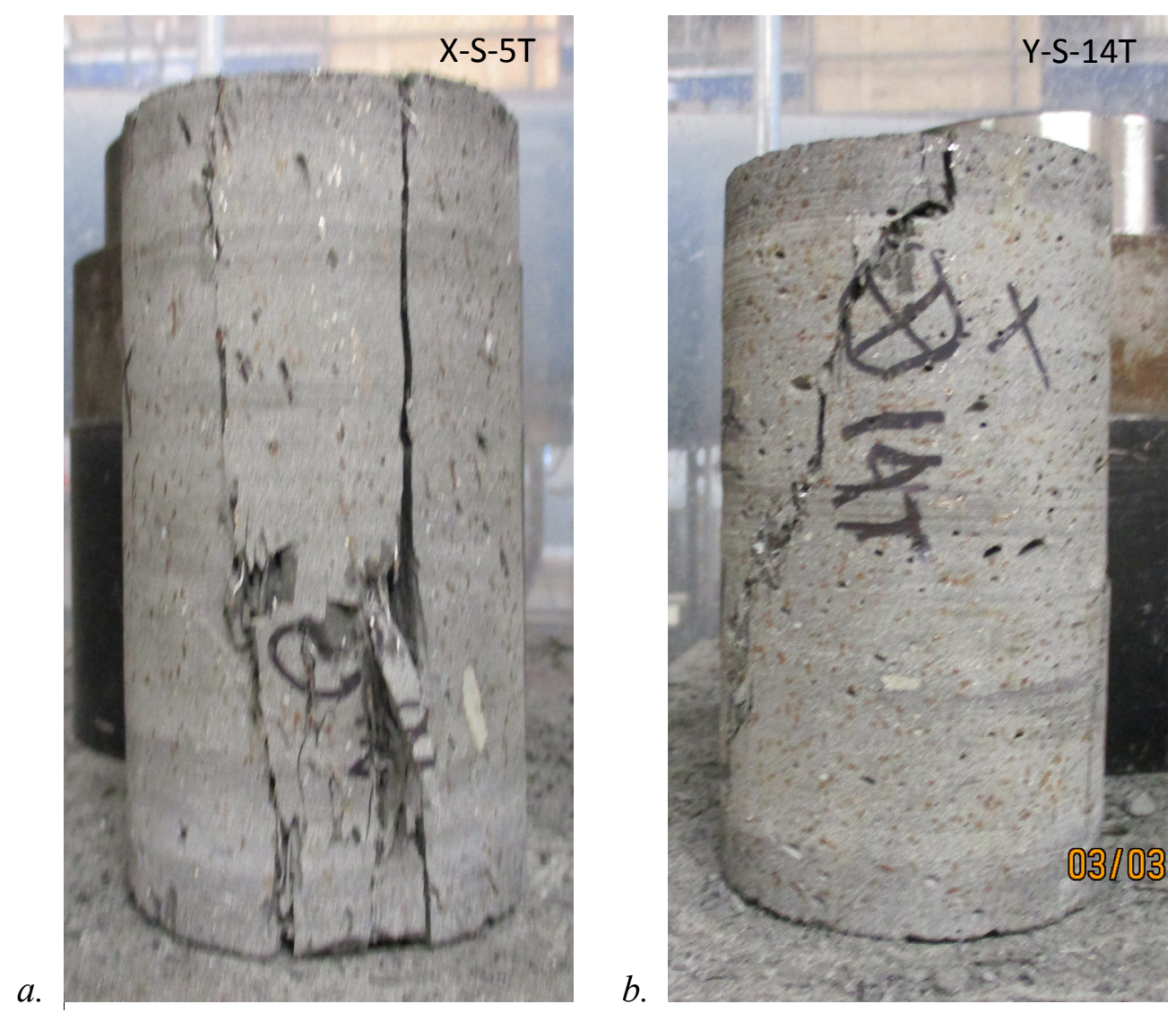

Figure 4.6. Quasistatic failure modes: $a$. columnar failure of specimen X-S-5T $b$. shear/side fracture of specimen Y-S-14T. 
The tests reported in Table 4.3 were conducted with neoprene bearing pads. Six additional tests were carried out to determine the effect of end conditions. The concrete age in these later tests was 364 days (52 weeks). Tests were conducted with the specimens in direct contact with steel bearing surfaces, and results are shown in Table 4.4. The mean compressive strength when bearing on steel was $26.62 \mathrm{ksi}$, compared to $17.42 \mathrm{ksi}$ when bearing on neoprene pads. Therefore, on average, the tests on neoprene pads gave a strength that was lower by $35 \%$.

Table 4.4. Quasistatic compressive strength of Cor-Tuf specimens bearing on steel*.

\begin{tabular}{|c|c|c|c|c|}
\hline \multirow{2}{*}{$\begin{array}{c}\text { Specimen } \\
\text { ID }\end{array}$} & \multirow{2}{*}{$\begin{array}{c}\text { Peak load } \\
\text { (lbf) }\end{array}$} & \multirow{2}{*}{$\begin{array}{c}\text { Compressive } \\
\text { strength, } \\
\boldsymbol{f}_{c s}(\mathrm{ksi})\end{array}$} & \multicolumn{2}{|r|}{ Failure mode } \\
\hline & & & Type & Remarks \\
\hline $\mathrm{X}-\mathrm{S}-1 \mathrm{~T}$ & 159,942 & 22.29 & 2 & Cone-and-split \\
\hline Y-S-1B & 187,920 & 26.42 & 4 & Predominantly shear \\
\hline Z-S-2T & 184,327 & 25.90 & 1 & Cone \\
\hline Z-S-15B & 177,440 & 24.72 & 2 & Cone-and-split \\
\hline Y-S-16T & 218,231 & 30.42 & 1 & Cone \\
\hline Z-S-16M & 214,766 & 29.99 & 1 & Cone \\
\hline & Mean & 26.62 & & \\
\hline & St Dev & 3.12 & & \\
\hline & $\mathrm{COV}$ & $11.7 \%$ & & \\
\hline
\end{tabular}

*Cored specimens tested without neoprene pads, age $=364$ days.

Different end conditions resulted in different stress distributions in the specimens: this is evident from the failure modes when tested on neoprene pads (mainly columnar and side fractures) and those when tested on steel (mainly cone and cone-and-split fractures). The efficiency of fiber reinforcement depends on its orientation with respect to the crack. Thus, the same fiber orientation (relative to the axis of loading) would likely have different strengthening effects with the two end conditions. Section 5.3.1 addresses the different stress distributions, and how this issue is handled in the analysis. 
Finally, results from quality control testing performed at ERDC are given in Table 4.5. Three 4 in. by 8 in. cylinders were cast at the same time as the beam and tested for 28-day compressive strength by ERDC staff. These cylinders received the same cure treatment at the beam and were stored in ambient conditions until testing. The first cylinder was damaged during demolding, leading to a lower compressive strength. The two undamaged cylinders had strengths of 28-29 ksi, as would be expected for Cor-Tuf.

Table 4.5. Quasistatic compressive strength of Cor-Tuf control specimens*.

\begin{tabular}{|c|c|c|c|c|}
\hline \multirow{2}{*}{$\begin{array}{c}\text { Specimen } \\
\text { ID }\end{array}$} & \multirow{2}{*}{$\begin{array}{c}\text { Peak load } \\
\text { (lbf) }\end{array}$} & \multirow{2}{*}{$\begin{array}{c}\text { Compressive } \\
\text { strength, } \\
\boldsymbol{f}_{c}^{\prime} \text { (ksi) }\end{array}$} & \multicolumn{2}{|r|}{ Failure mode } \\
\hline & & & Type & Remarks \\
\hline 1 & 312,790 & 24.64 & 2 & $\begin{array}{l}\text { Cone-and-split (side damaged } \\
\text { prior to test) }\end{array}$ \\
\hline 2 & 358,840 & 28.27 & 1 & Cone \\
\hline 3 & 376,650 & 29.68 & 1 & Cone \\
\hline & Mean & 27.53 & & \\
\hline & St Dev & 2.60 & & \\
\hline & $\mathrm{COV}$ & $9.4 \%$ & & \\
\hline
\end{tabular}

$*$ Cylinders tested without neoprene pads, age $=28$ days. 


\subsection{Dynamic Compression Testing}

The results of dynamic compression testing are summarized in Table 4.6. Results presented are the average of three wave analyses on the $\mathrm{A} / \mathrm{B}$ and $\mathrm{C} / \mathrm{D}$ strain gage channels. Specimens X-D-6B, X-D-11T (only one side), and Y-D-13T were still essentially intact after testing.

Table 4.6. Dynamic compressive strength of Cor-Tuf specimens.

\begin{tabular}{|c|c|c|c|c|c|}
\hline \multirow{2}{*}{$\begin{array}{l}\text { Specimen } \\
\text { ID }\end{array}$} & \multirow{2}{*}{$\begin{array}{c}\text { Peak } \\
\text { stress, } \\
\boldsymbol{f}_{c d} \\
(\mathbf{k s i})\end{array}$} & \multirow{2}{*}{$\begin{array}{l}\text { Strain at } \\
\text { peak } \\
\text { stress, } \varepsilon_{c r} \\
\text { (in./in.) }\end{array}$} & \multirow{2}{*}{$\begin{array}{l}\text { Max strain } \\
\text { rate, } \dot{\varepsilon}_{\text {max }} \\
\quad\left(\mathrm{s}^{-1}\right)\end{array}$} & \multicolumn{2}{|r|}{ Failure mode } \\
\hline & & & & Type & Remarks \\
\hline X-D-2M* & 40.00 & 0.0120 & 168 & 2 & Cone-and-split \\
\hline Y-D-2B & 48.62 & 0.0126 & 172 & 3 & Columnar $^{\dagger}$ \\
\hline $\mathrm{X}-\mathrm{D}-3 \mathrm{~T}^{*}$ & 50.02 & 0.0122 & 170 & 3 & Columnar \\
\hline Z-D-3M & 50.15 & 0.0129 & 181 & 3 & Columnar \\
\hline Z-D-4T* & 54.73 & 0.0122 & 172 & 3 & Columnar \\
\hline Y-D-4B & 48.69 & 0.0121 & 167 & 3 & Columnar \\
\hline Y-D-5M** & 38.12 & 0.0118 & 196 & 4 & Shear with side fracture ${ }^{\dagger}$ \\
\hline Z-D-5B & 41.04 & 0.0124 & 185 & 3 & Columnar \\
\hline X-D-6B & 56.57 & 0.0105 & 136 & 3 & $\begin{array}{l}\text { Thin columnar fracture } \\
\text { on either side }\end{array}$ \\
\hline Y-D-7T & 42.84 & 0.0131 & 184 & 2 & Cone-and-split \\
\hline X-D-9T & 44.87 & 0.0115 & 166 & 4 & Shear $^{\dagger}$ \\
\hline Z-D-9M** & 45.58 & 0.0126 & 187 & 3 & Columnar \\
\hline Z-D-10T* & 51.19 & 0.0120 & 172 & 3 & Columnar \\
\hline Y-D-10B* & 45.62 & 0.0129 & 172 & 3 & Columnar \\
\hline X-D-11T* & 58.54 & 0.0119 & 173 & 3 & Columnar \\
\hline Z-D-11B & 52.51 & 0.0125 & 181 & 2 & Cone-and-split \\
\hline $\mathrm{X}-\mathrm{D}-12 \mathrm{M}^{*}$ & 49.05 & 0.0122 & 164 & 3 & Columnar \\
\hline Y-D-13T* & 52.65 & 0.0113 & 145 & 6 & Side fracture \\
\hline Mean & 48.38 & 0.0121 & 172 & & \\
\hline St Dev & 5.68 & 0.0006 & 14 & & \\
\hline $\mathrm{COV}$ & $11.7 \%$ & $5.2 \%$ & $8.2 \%$ & & \\
\hline
\end{tabular}

*Departure from parallelness greater than 0.5 degree parallelness tolerance.

**Departure from parallelness greater than 1.0 degrees.

†Very few fibers in a failure surface. 
Several frames from high speed video of testing specimen Y-D-4T are shown in Figure 4.7. Each frame is numbered, and the elapsed time from frame 0 is listed. The resolution is only $256 \times 256$ pixels at $26,000 \mathrm{fps}$; a high speed camera can only process so many pixels' worth of data per second, so increasing the frame rate means sacrificing resolution. For reference, the input bar appears on the right side of each frame, and the output bar on the left. Frame 0 shows the specimen just prior to cracking. In frame 1, cracking initiates at voids on the output bar side. A hairline crack propagates toward the input bar in frame 2, with additional cracking/crushing at the output bar. Two hairline cracks across the specimen length are present by frame 3. Spalling in the upper left corner also occurs at this time. Most cracks have formed by frame 4, and fine particles are beginning to be ejected from the cracks. Jumping forward in time to frame 39, the fragments have noticeably separated and are falling from between the bars. Note that stress and strain measurements may be inaccurate once the specimen begins to separate from the bars. This is because the SHPB technique relies on wave propagation to measure the specimen's response.

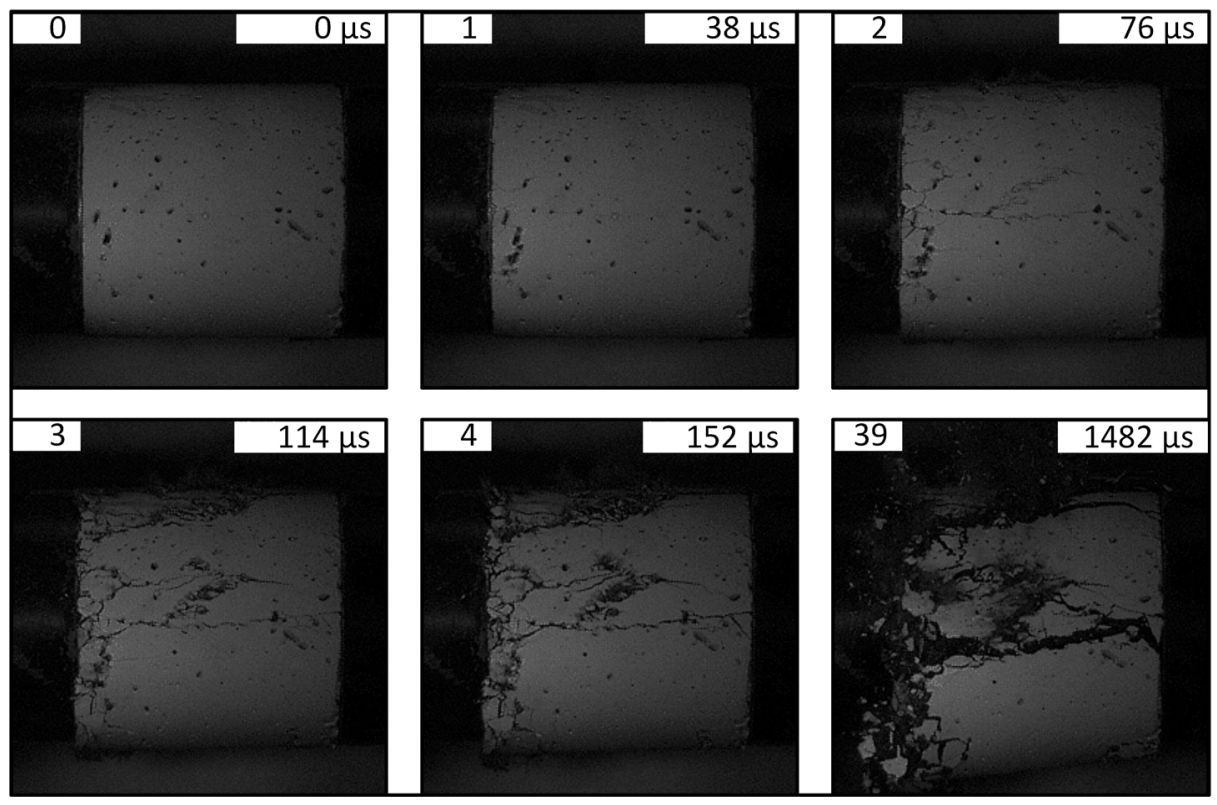

Figure 4.7. High speed video frames showing failure of specimen Y-D-4T. Each frame is numbered in the upper left hand corner. The approximate time relative to frame 0 is given in the upper right hand corner. 
Additional selected images from high speed video of testing are presented in Figure 4.8. As before, the input bar appears on the right side of each frame, and the output bar on the left. The majority of specimens failed with columnar fractures. Specimen X-D-3T (Figure 4.8a) is shown as an example of columnar failure. Columnar failures were often accompanied by crushing at either the input or output bar; in this case, crushing occured at the input bar. Specimen Y-D-5M (Figure 4.8b) was a peculiar case, as it failed on a fairly clean shear plane and split into two wedge-like halves, one of which broke the plexiglass shield in front of the camera. The shear plane can be seen on the front of the specimen near the input bar, and extends toward the rear of the specimen at the output bar. Specimen Y-D-7T (Figure 4.8c) showed a cone-and-split failure, with the cone on the input bar side. Finally, specimen Y-D-13T (Figure 4.8d) experienced only side fractures, and remained intact after the test.

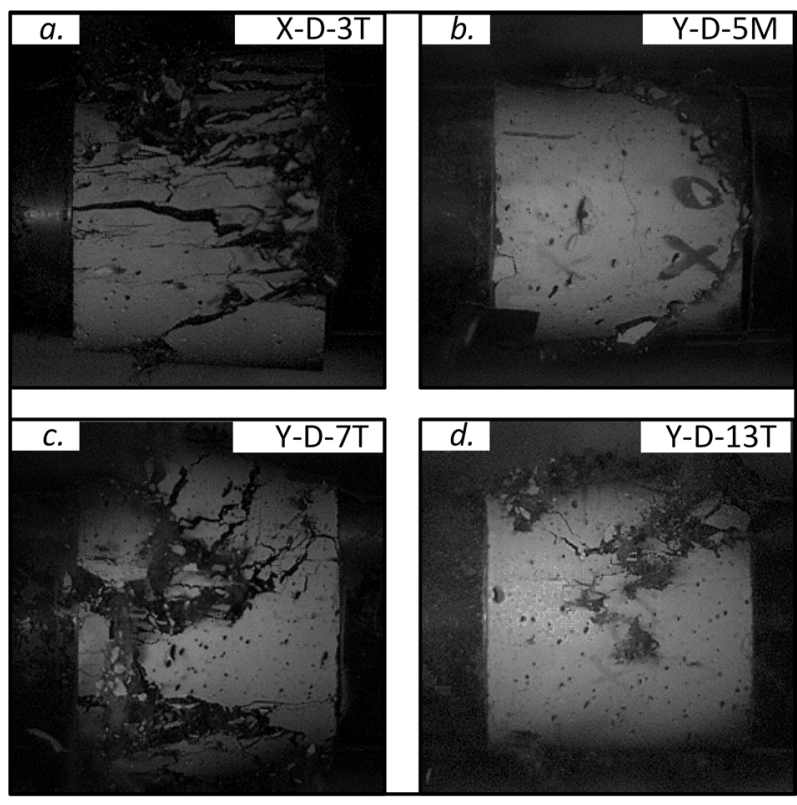

Figure 4.8. High speed images of failures $a$. specimen X-D-3T $b$. specimen Y-D-5M $c$. specimen Y-D-7T $d$. specimen Y-D-13T. 
Intact specimens had minor loss of material and cracks that were bridged effectively by fibers. The three specimens that were intact after dynamic compression testing are shown in Figure 4.9. Specimen X-D-6B was essentially intact, with a small columnar fracture on either side. Specimen X-D-11T also exhibited a columnar fracture, though only on one side. The right side of the specimen in the figure is still fairly intact. There was some difficulty in classifying specimen Y-D-13T, which did not exhibit a well-formed cone, despite initial appearances. The cracks shown do not extend through to the other side, and so were considered a side fracture. It appears that fiber crackbridging helped restrain crack growth.
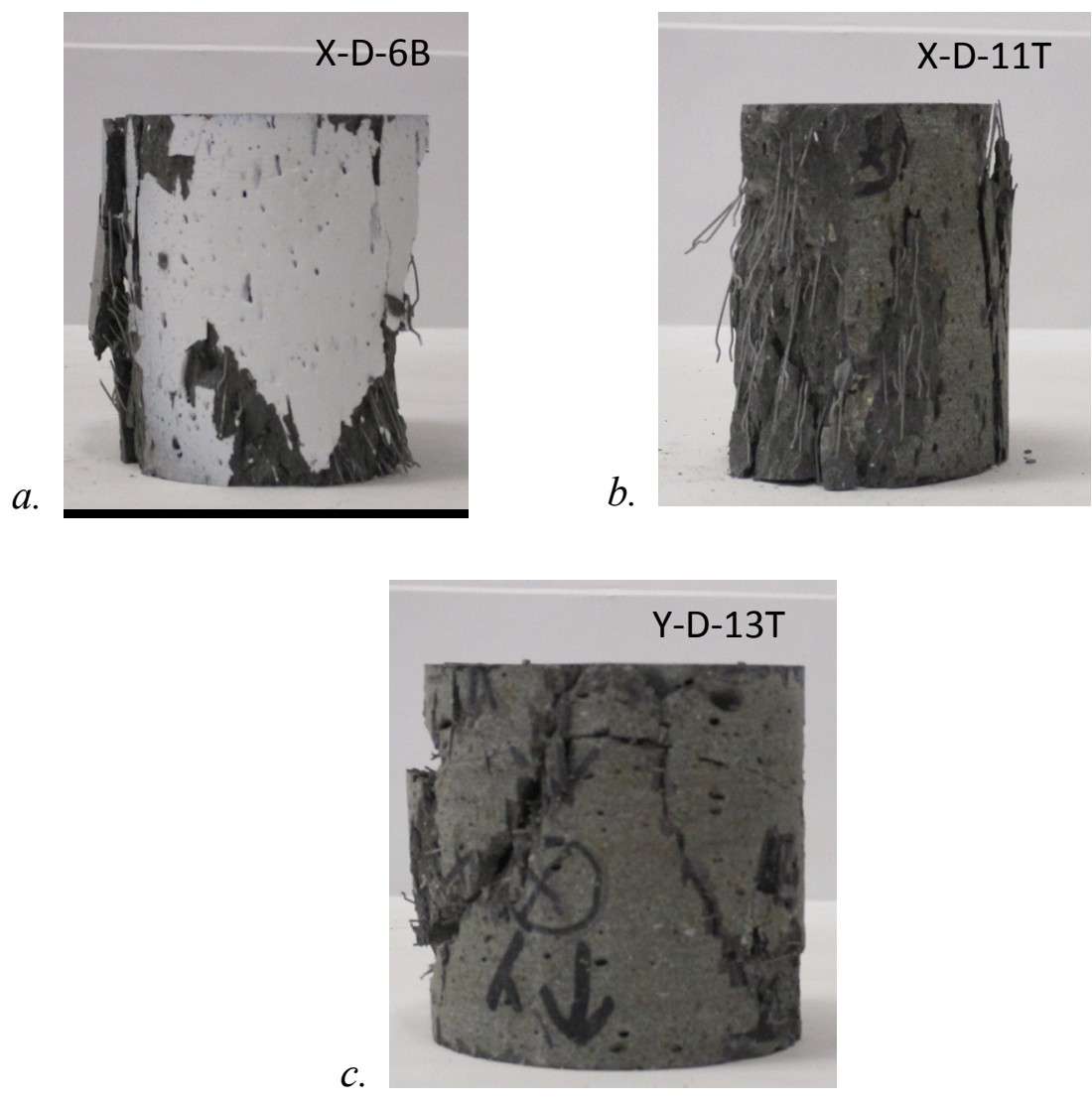

Figure 4.9. Intact specimens $a$. specimen X-D-6B, small columnar fractures $b$. specimen X-D-11T, columnar fracture on one side only $c$. specimen Y-D-13T, side fractures bridged by fibers. 
ASTM failure type 2 (ASTM 2015), cone-and-split, had a slightly truncated appearance in the specimens tested. Two examples of this wedge-like failure are shown in Figure 4.10. The altered failure pattern may be due to the use of shorter specimens, which have an aspect ratio of 1, compared to the aspect ratio of 2 for which the failure mode descriptions were developed. The presence of fibers likely also plays a role in altering the fracture pattern, and are not included in the failure mode descriptions (ASTM 2015). While failure descriptions for NSC can be applied to UHPC, it seems that a classification system specifically designed for UHPC would be better able to describe the types of failures that were observed. Developing such a classification is beyond the scope of this work, however.
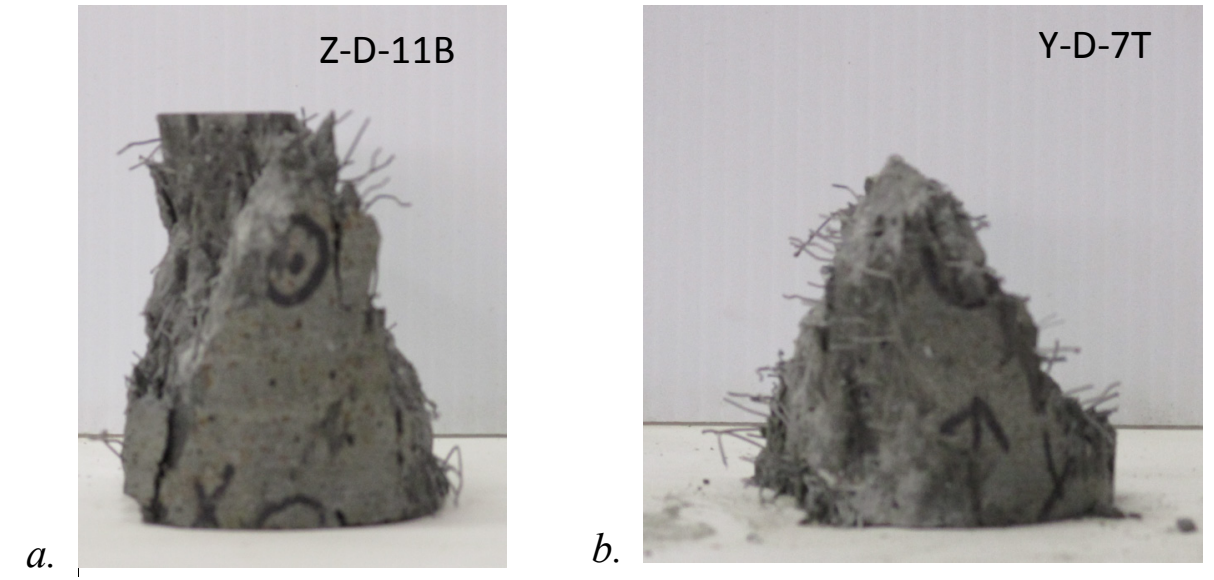

Figure 4.10. Examples of truncated type 2 failures. 


\section{CHAPTER 5 DISCUSSION}

\subsection{Random and Aligned Fiber Orientations}

To make sense of the various summary statistics for fiber orientation, it is useful to consider the values that would occur for randomly-oriented fibers. However, it is first necessary to clarify what is meant by randomly-oriented. Consider a fiber free to rotate about its center of gravity. The ends of the fiber describe a sphere of radius $\ell / 2$, but only one end (and the corresponding hemisphere of radius $\ell / 2$ ) need be considered, as the position of one end determines that of the other. If the fiber end is equally likely to fall at any point on the hemisphere, then the probability density function is $f(\theta)=\sin (\theta)$, where $\theta$ is measured from the axis through the hemisphere's pole. Figure 5.1 shows the probability density function for the orientation angle. For a derivation, see, for example, Victor C. Li et al. (1991). In dealing with probabilities, remember that angles must be expressed in radians.

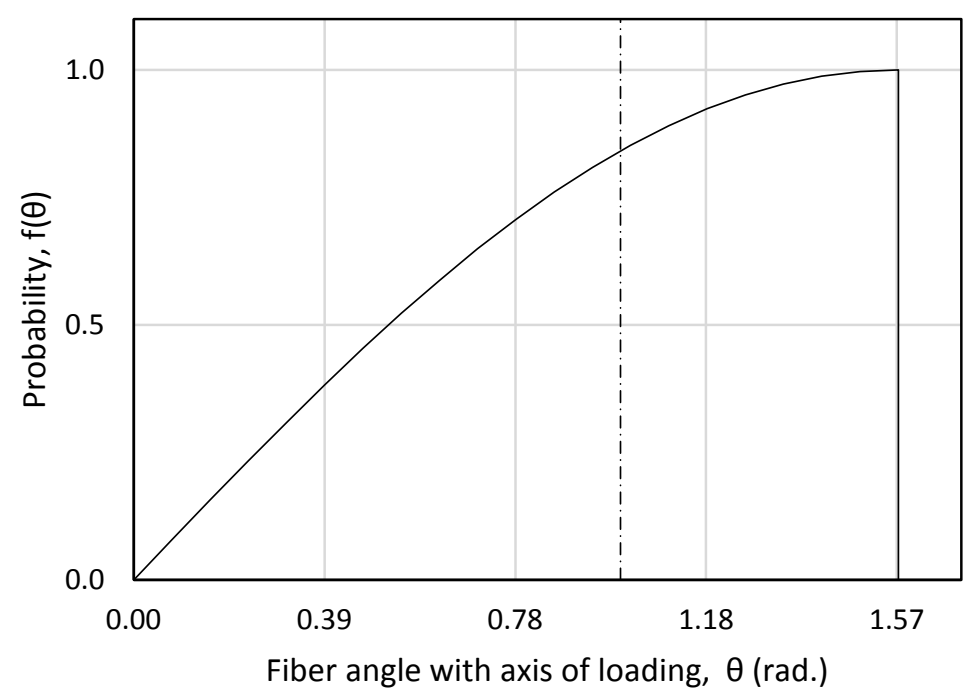

Figure 5.1. Theoretical probability density function for fiber orientation angle. The mean orientation angle is shown with a dash-dot line. 
The mean orientation angle is shown in the figure by a dash-dot line, and is 1 radian or about 57.3 degrees. The standard deviation is exactly $\sqrt{\pi-3}$ radians or 21.6 degrees. Finally, the mode is $\pi / 2$ radians or 90 degrees. Now, these can be compared to the results in Table 4.1. Generally, the summary statistics for the $z$-direction orientation angles are closest to what would be obtained for randomly-oriented fibers. The mean $y$ direction orientation angle (71.2 degrees) is higher than the mean for randomly-oriented fibers. This indicates a slightly greater tendency to angles oriented at 90 degrees from the $y$-axis. The mean $x$-direction orientation angle (39.0 degrees) is lower than the mean for randomly-oriented fibers, indicating a greater tendency to angles oriented at 0 degrees from the $x$-axis, that is to say, aligned with the $x$-axis.

The cumulative distribution function for the probability density function (Figure $5.1)$ is given by:

$$
\begin{aligned}
F(\theta) & =\int_{-\infty}^{\theta} f(z) \mathrm{d} z=\int_{0}^{\theta} \sin (z) \mathrm{d} z \\
& =1-\cos (\theta) .
\end{aligned}
$$

This cumulative distribution function is shown in Figure 5.2.

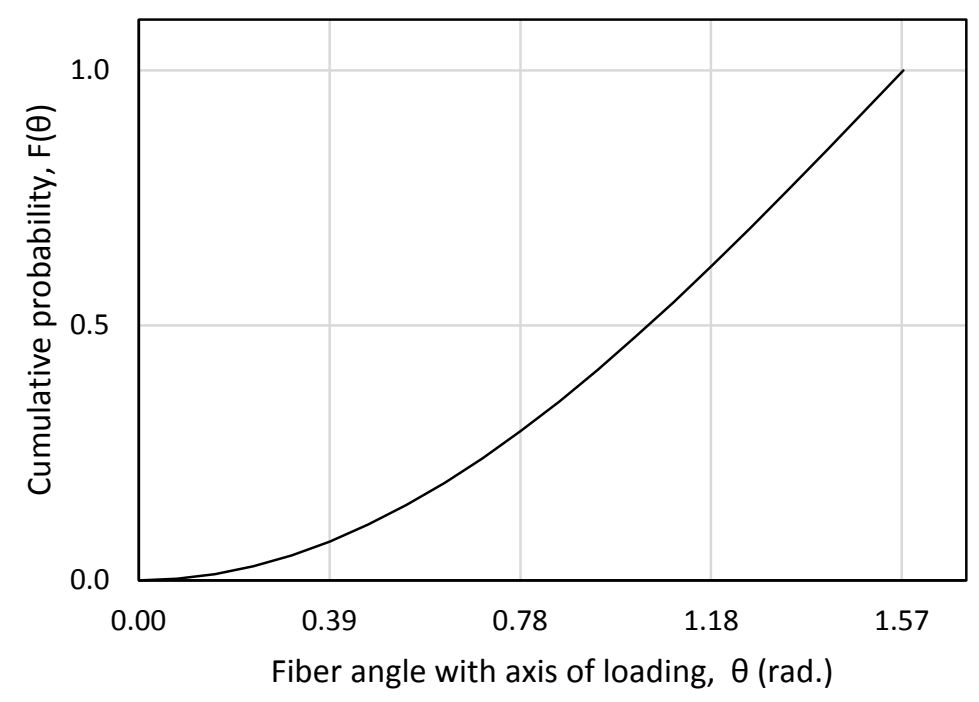

Figure 5.2. Theoretical cumulative distribution function for fiber orientation angle. 
Representative histograms of $x$-, $y$-, and $z$-direction orientation angles are shown in Figure 5.3. The early peak for the $x$-axis histogram (Figure 5.3a) is consistent with alignment along the $x$-axis. The $y$-axis histogram (Figure 5.3b) has a long left tail, with most of the fibers taking on higher orientation angles. Note that the peak value on this histogram is about 3 times that of the other two: the angles are tightly grouped, hence the low standard deviations for the $y$-axis in Table 4.1. Finally, the $z$-axis histogram (Figure 5.3c) falls somewhere between the between the other two. Fibers have mainly higher orientation angles, but are not as tightly grouped as for the $y$-axis.

The histograms cannot be directly compared to either the probability density function or the cumulative distribution function. In a histogram, the frequency for a bin spanning $\left[\theta_{a}, \theta_{b}\right]$ is $\int_{\theta_{a}}^{\theta_{b}} f(z) \mathrm{d} z$, assuming that the sample is representative. Because of this, the histograms can be easily converted into a cumulative distribution function. (Note that this is not a cumulative mass function: the plot is discretized, but the underlying variable - the orientation angle - is continuous, not discrete.) Bar plots of the cumulative distribution function are shown in Figure 5.4. The cumulative distribution function for the $x$-axis (Figure 5.4a) initially grows more quickly than the theoretical curve, then more slowly. The cumulative distribution function for the $y$-axis (Figure $5.4 b$ ) grows slowly until rapidly increasing between 50 and 60 degrees. Finally the cumulative distribution function for the $z$-axis (Figure 5.4c) most closely resembles the theoretical curve shown in Figure 5.2. This indicates that fibers are nearly randomly oriented with respect to the $z$-axis, and less so with respect to the $x$ - and $y$-axes. 


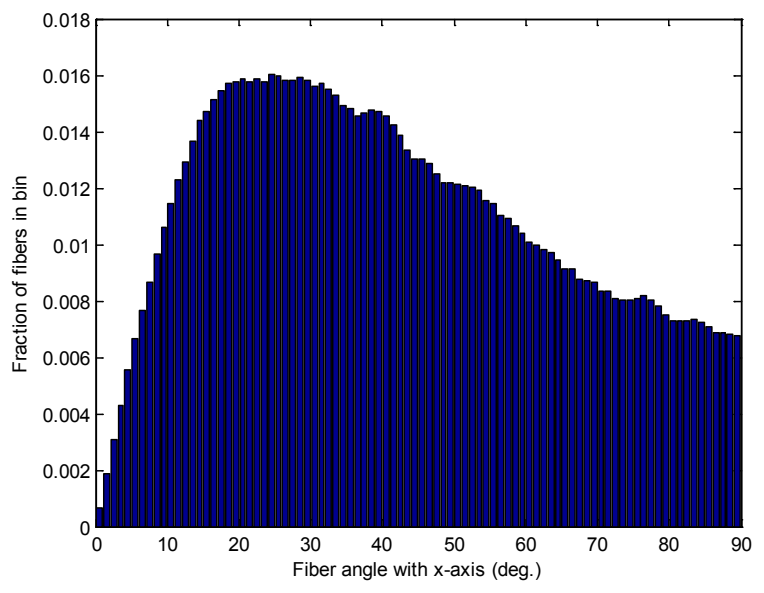

a.

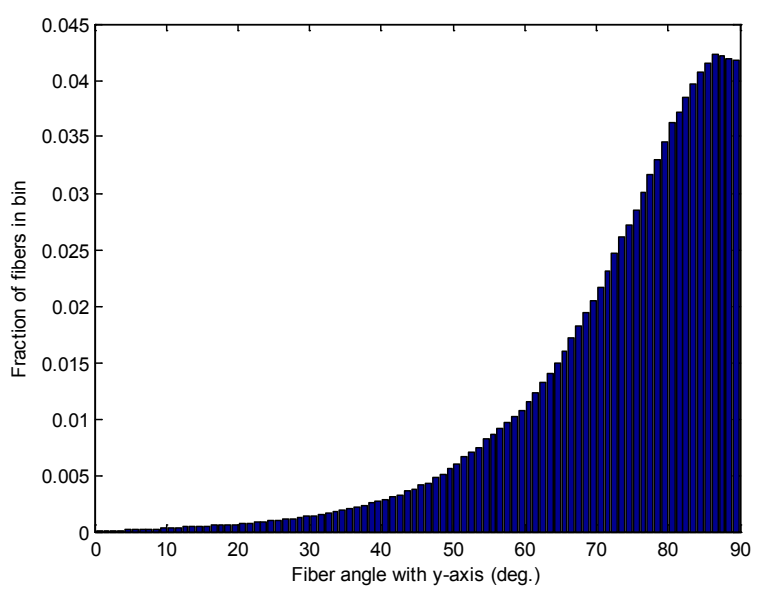

$b$.

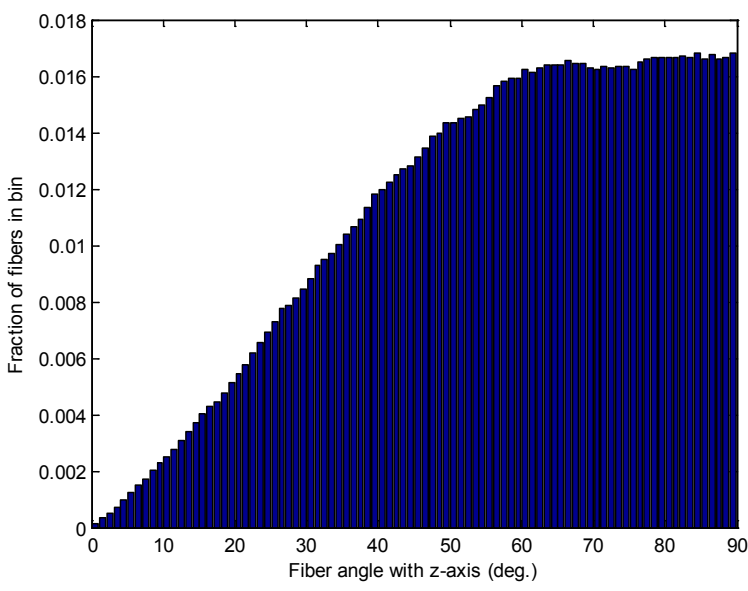

Figure 5.3. Measured orientation angle distribution for specimen X-D-2M for $a$. the $x$-axis, $b$. the $y$-axis, and $c$. the $z$-axis. 


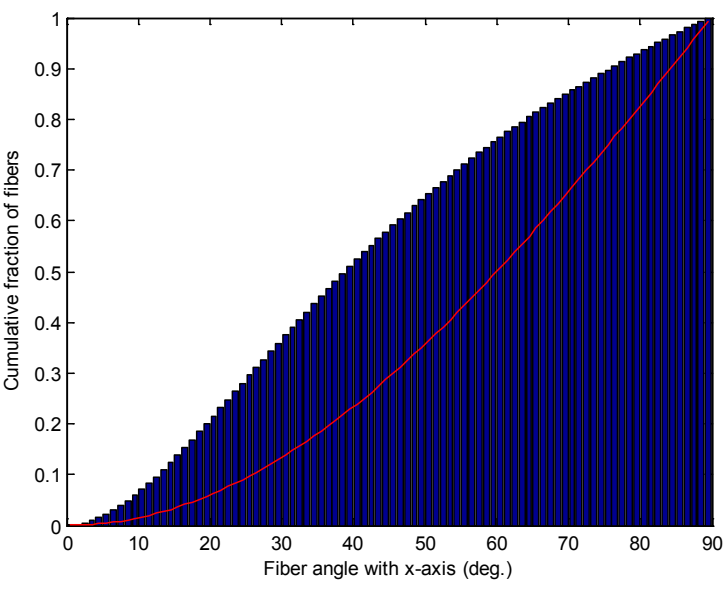

$a$.

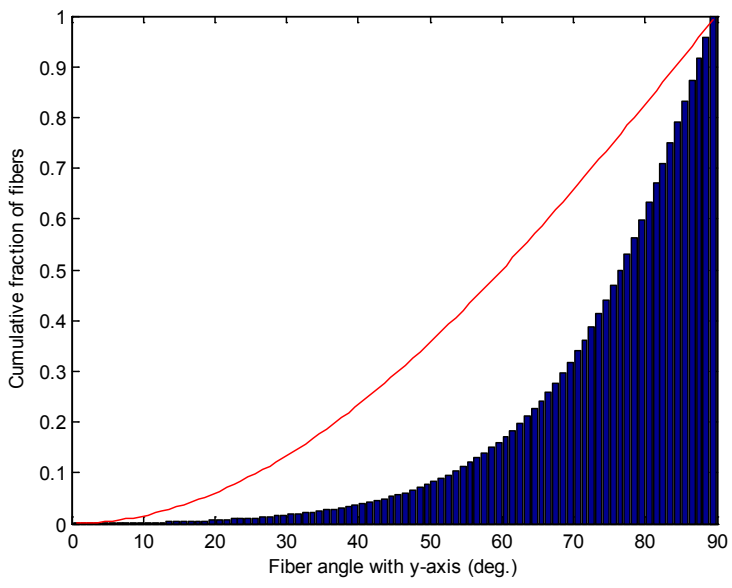

$b$.

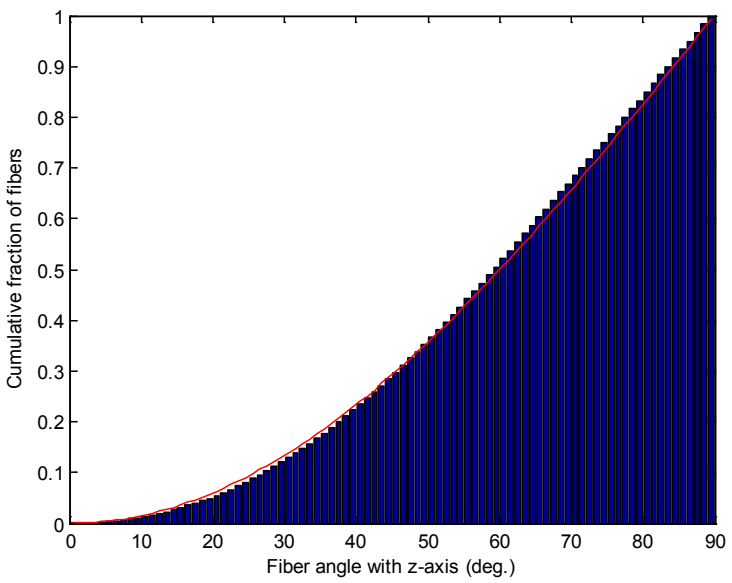

Figure 5.4. Cumulative distribution of orientation angle for specimen X-D-2M for $a$. the $x$-axis, $b$. the $y$-axis, and $c$. the $z$-axis. The theoretical curve for a randomly-oriented fiber is shown in red. 


\subsection{Variation of Fiber Orientation throughout Beam}

It was expected that alignment due to flow would result in an $x$-direction average orientation angle, $\bar{\theta}_{x}$, that differed from both the $y$ - and $z$-direction average orientation angles, $\bar{\theta}_{y}$ and $\bar{\theta}_{z}$, respectively. This was assessed using the Games-Howell method for simultaneous comparison in Minitab 17 Statistical Software (Minitab 2010). The GamesHowell method can be used when the variances of samples are unequal, and also does not require equal sample sizes. Results of this statistical test at a $95 \%$ confidence level ( $\alpha=$ $0.05)$ are shown in Table 5.1. The number of samples for $\bar{\theta}_{x}, \bar{\theta}_{y}$, and $\bar{\theta}_{z}$ was 31 . The $95 \%$ confidence interval (CI) is given for each difference: if the CI does not include zero, the difference is significant. The differences $\bar{\theta}_{y}-\bar{\theta}_{x}$ and $\bar{\theta}_{z}-\bar{\theta}_{x}$ are positive, indicating that the average orientation angle in the $x$-direction is less than that in either the $y$ - or $z$ direction. This is consistent with alignment in the $x$-direction. The more closely aligned a fiber is with the $x$-axis, the lower its orientation angle will be with that axis. If the fiber has a low $x$-direction orientation angle, then its $y$ - and $z$-direction orientation angles must be high. The magnitude of the difference $\bar{\theta}_{z}-\bar{\theta}_{y}$ is less than for the other two comparisons, as the average orientation angles in the $y$ - and $z$-directions do not differ from each other as much as they differ from those in the $x$-direction. Overall, the average orientation angles are significantly different in each direction.

Table 5.1. Results of Games-Howell comparison for average orientation angle.

\begin{tabular}{cccc}
\hline Comparison & $\begin{array}{c}\text { Difference of means } \\
\text { (degrees) }\end{array}$ & $\begin{array}{c}\text { 95\% CI for difference } \\
\text { (degrees) }\end{array}$ & Adjusted $\boldsymbol{p}$-value \\
\hline $\bar{\theta}_{y}-\bar{\theta}_{x}$ & 32.17 & $(28.91,35.43)$ & $<0.001$ \\
$\bar{\theta}_{z}-\bar{\theta}_{x}$ & 23.15 & $(19.81,26.48)$ & $<0.001$ \\
$\bar{\theta}_{z}-\bar{\theta}_{y}$ & -9.02 & $(-11.50,-6.55)$ & $<0.001$ \\
\hline \hline
\end{tabular}

It appeared that the fibers were most aligned in the $x$-direction near the top and bottom of the form. The significance of height on the average $x$-direction orientation angle was assessed using the Games-Howell method for simultaneous comparison in Minitab. Results of this statistical test at a 95\% confidence level $(\alpha=0.05)$ are shown in 
Table 5.2. The number of samples was 9 for the bottom third, and 11 for both the middle and top thirds. The test indicates that the middle third differs significantly from both the bottom and top thirds, confirming that fibers were most aligned in the $x$-direction near the top and bottom of the form. The top and bottom thirds are not significantly different. As previously mentioned, wall effects are present at the bottom, causing the fibers to align parallel to the formwork surface. However, the bottom 2 in. was avoided when taking cores, so wall effects should not influence the orientation in the specimens. At the top, it is possible that the fibers were disturbed during placement: when the flowability of the concrete decreased, it had to be hand-guided to fill the remainder of the form. On the whole, it seems that fibers have a reduced tendency to align in the direction of flow at the midheight of the beam. This is likely related to the flow process when the beam was cast, but is outside the scope of this work.

Table 5.2. Results of Games-Howell comparison for average $\boldsymbol{x}$-direction orientation angle.

\begin{tabular}{cccc}
\hline \hline Comparison & $\begin{array}{c}\text { Difference of means } \\
\text { (degrees) }\end{array}$ & $\begin{array}{c}\text { 95\% CI for difference } \\
\text { (degrees) }\end{array}$ & Adjusted $\boldsymbol{p}$-value \\
\hline Middle - Bottom & 12.06 & $(8.12,16.01)$ & $<0.001$ \\
Top - Bottom & 2.17 & $(-1.44,5.79)$ & 0.297 \\
Top - Middle & -9.89 & $(-14.31,-5.48)$ & $<0.001$ \\
\hline \hline
\end{tabular}

Finally, the degree of alignment in each direction was assessed by comparing the variance of the average orientation angles, $\operatorname{Var}(\bar{\theta})$. A test for equal variance using the multiple comparisons method was performed in Minitab. Results of the statistical test at a 95\% confidence level $(\alpha=0.05)$ are shown in Table 5.3. The number of samples for $\bar{\theta}_{x}, \bar{\theta}_{y}$, and $\bar{\theta}_{z}$ was 31 . For this test, the null hypothesis is that all variances are equal; the corresponding alternate hypothesis is that at least one variance is different. The associated $p$-value is 0.036 , so at least one variance is significantly different for $\alpha=0.05$. Results show that $\operatorname{Var}\left(\bar{\theta}_{x}\right)$ and $\operatorname{Var}\left(\bar{\theta}_{y}\right)$ are significantly different, as the CIs for the standard deviation do not overlap. This indicates that the average orientation angle is more variable in the $x$-direction than in the $y$-direction. So, although fibers are aligned in 
the $x$-direction, they show varying degrees of alignment. This may be due to turbulence in the flow, though if turbulence were responsible, the variance at the casting end of the beam should differ more from the variance at the other end. A similar trend would be expected for the mean. Referring back to Figure 4.3, Figure 4.4, and Figure 4.5, this does not appear to be the case: the variance and mean do not show any clear trend with distance from the casting end. Lastly, $\operatorname{Var}\left(\bar{\theta}_{z}\right)$ is not significantly different from $\operatorname{Var}\left(\bar{\theta}_{x}\right)$ or $\operatorname{Var}\left(\bar{\theta}_{y}\right)$.

Table 5.3. Results of multiple comparisons method for equal variance.

\begin{tabular}{cccc}
\hline \hline Sample & $\begin{array}{c}\text { Estimated standard } \\
\text { deviation (degrees) }\end{array}$ & $\begin{array}{c}\text { 95\% CI for standard } \\
\text { deviation (degrees) }\end{array}$ & Differs from \\
\hline $\bar{\theta}_{x}$ & 6.44 & $(5.21,8.59)$ & $\bar{\theta}_{y}$ \\
$\bar{\theta}_{y}$ & 3.85 & $(3.17,5.07)$ & $\bar{\theta}_{x}$ \\
$\bar{\theta}_{z}$ & 4.24 & $(3.68,5.28)$ & - \\
\hline
\end{tabular}

Multiple comparisons $p=0.036$

\subsection{Orientation Effects}

Previously, orientation angles were presented in degrees, using the mean and pseudomode to characterize the central tendency, and the standard deviation to assess the alignment. Dealing with orientation angles is useful for understanding the orientation of fibers within the beam, but the orientation number has a firmer basis for examining material properties. Building on the definition from Section 2.2.1, two orientation numbers can be defined as below:

$$
\begin{aligned}
& \eta_{\|}=\frac{1}{N} \sum_{i=1}^{N} \cos \left(\theta_{i}\right), \\
& \eta_{\perp}=\frac{1}{N} \sum_{i=1}^{N} \sin \left(\theta_{i}\right)
\end{aligned}
$$


where: $\quad N=$ total number of fibers;

$$
\theta_{i}=\text { angle of fiber } i \text { with the axis of loading. }
$$

The first of these, $\eta_{\|}$, is the ratio of the fibers' projected length along the axis of loading, to the total fiber length. If the traditional orientation number $\eta$ is calculated at a section normal to the axis of loading, then $\eta$ is equivalent to $\eta_{\|}$. Similarly, $\eta_{\perp}$ is the ratio of the fibers' projected length normal to the axis of loading, to the total fiber length. The orientation numbers of two simple fibers are illustrated in Figure 5.5. Although it might seem that $\eta_{\|}^{2}+\eta_{\perp}^{2}=1$, that is not the case, as the orientation numbers are averages over all fibers. In this study, the value of the sum $\eta_{\|}^{2}+\eta_{\perp}^{2}$ ranged from 0.85 to 0.97 . Though these two measures of orientation angle are not quantitatively related, they do have a qualitative relation, as will be seen in the plots in the following sections.

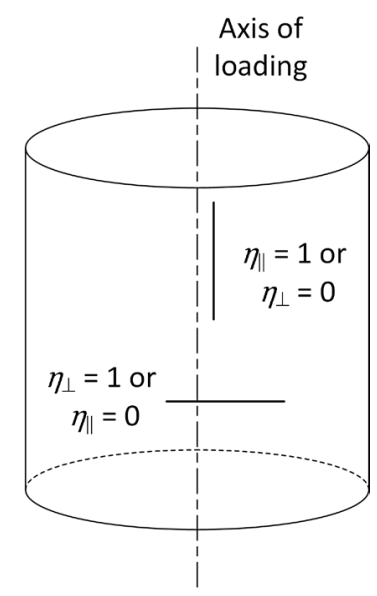

Figure 5.5. Illustration of parallel and perpendicular orientation number. For all specimens in this work, note that the axis of loading is the same as the direction in which they were cored. 


\subsubsection{Strength}

The variation of quasistatic compressive strength with orientation number is shown in Figure 5.6. The strengths are normalized by the maximum quasistatic compressive strength. Note that 15 data points are shown: 18 specimens were tested in quasistatic compression, but only 15 could be CT scanned. Orientation can be assessed using either $\eta_{\|}$or $\eta_{\perp}$ : quasistatic compressive strength is highest for low values of $\eta_{\|}$and high values of $\eta_{\perp}$. Because the strength enhancement is thought to be due to crack bridging by fibers perpendicular to the load, $\eta_{\perp}$ is a more natural choice of orientation parameter.

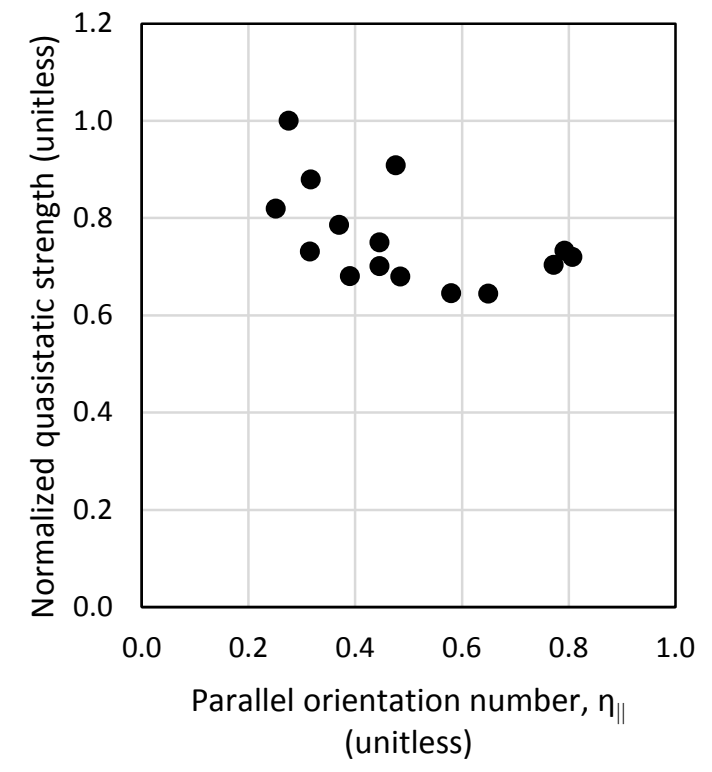

$a$.

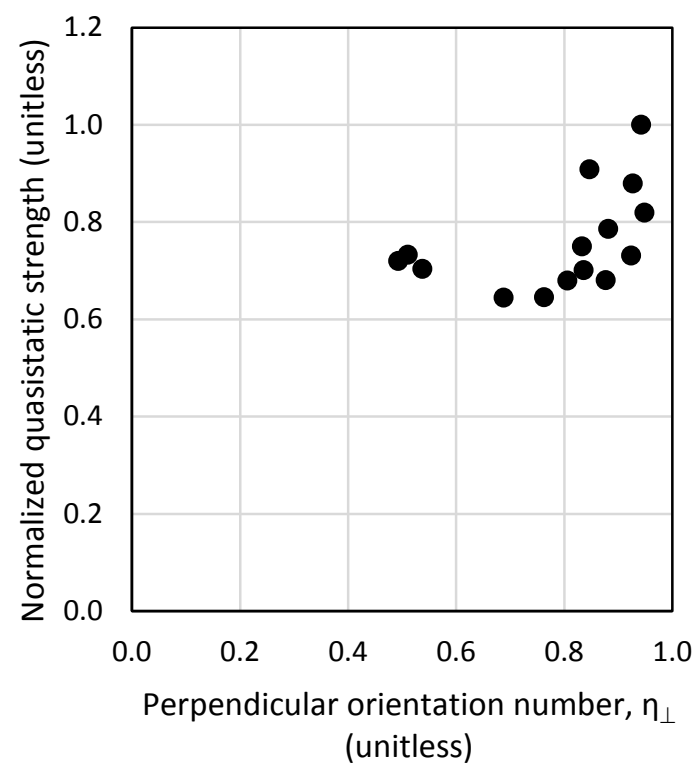

b.

Figure 5.6. Variation of quasistatic compressive strength with $a$. parallel orientation number and $b$. perpendicular orientation number.

Due to limited data for $\eta_{\perp}<0.8$, it is uncertain whether strength has a minimum near $\eta_{\perp} \approx 0.7$, or if the strength, in fact, increases monotonically with $\eta_{\perp}$. Under the first explanation, the low strengths are indicative of material performance when fibers are oriented at about 45 degrees relative to the load (roughly corresponding to $\eta_{\perp}=0.7$ ). Under the second explanation, the low strengths are due to experimental scatter. The two 
specimens with low strength, X-S-10M and X-S-13M, failed by side fracture and columnar fracture with diagonal cracking, respectively. These failure modes are shown in Figure 5.7. For a side fracture, it is possible that fibers could be aligned such that the fracture occurs on a weak plane where nearly all fibers are parallel to the crack. The angle with respect to the axis of loading does not provide enough information to tell if the fibers are parallel or perpendicular to the crack. If the crack pattern is known, the inplane angle can be used to distinguish between the two cases. However, it is difficult to predict the crack pattern (failure mode) prior to conducting a test. Further testing is needed to determine if strength always has a minimum near $\eta_{\perp} \approx 0.7$, and what effect the in-plane angle has.

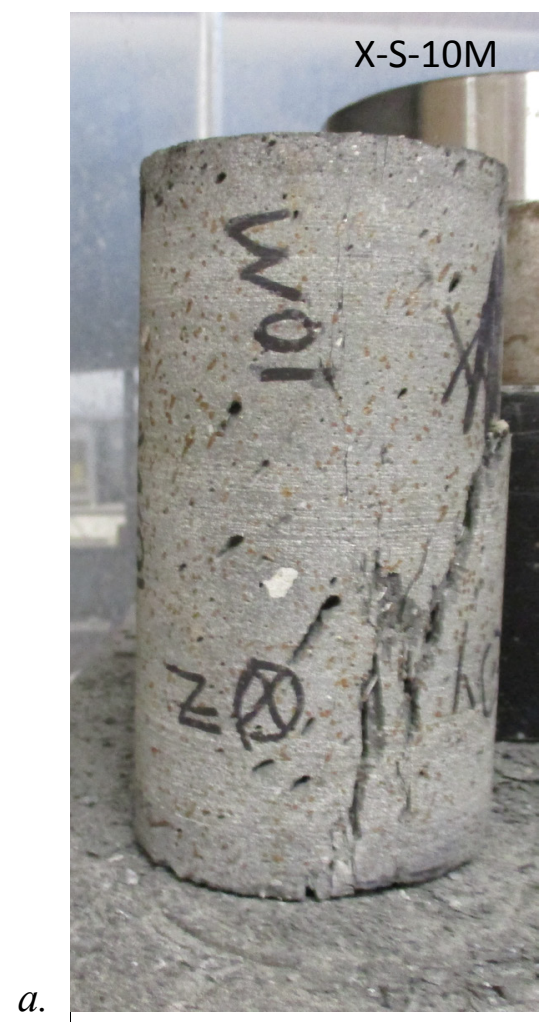

$b$.

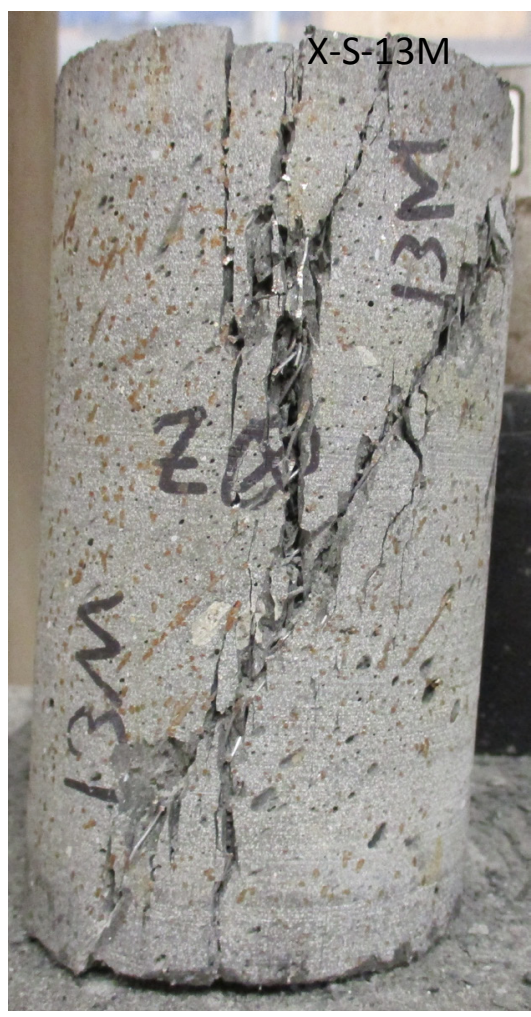

Figure 5.7. Specimens with low quasistatic strength: $a$. Specimen X-S-10T, side fracture $b$. specimen X-S-13M, columnar fracture with some side fracture tendency. 
To further investigate the interplay between failure mode and fiber orientation, the data from Figure 5.6b were replotted, with each point identified as a columnar failure or "other" failure. This plot is shown in Figure 5.8. Curiously, the "other" failures display an essentially linear trend with $\eta_{\perp}$, whereas the columnar failures suggest a parabolic trend. As previously discussed, it is not clear why this might be. One possible explanation is illustrated in Figure 5.9. Both of the fibers shown have an angle of 45 degrees with the axis of loading, but their in-plane angles are different. Fiber A bridges the crack, but fiber B is parallel to the crack and has little, if any, crack-bridging ability.

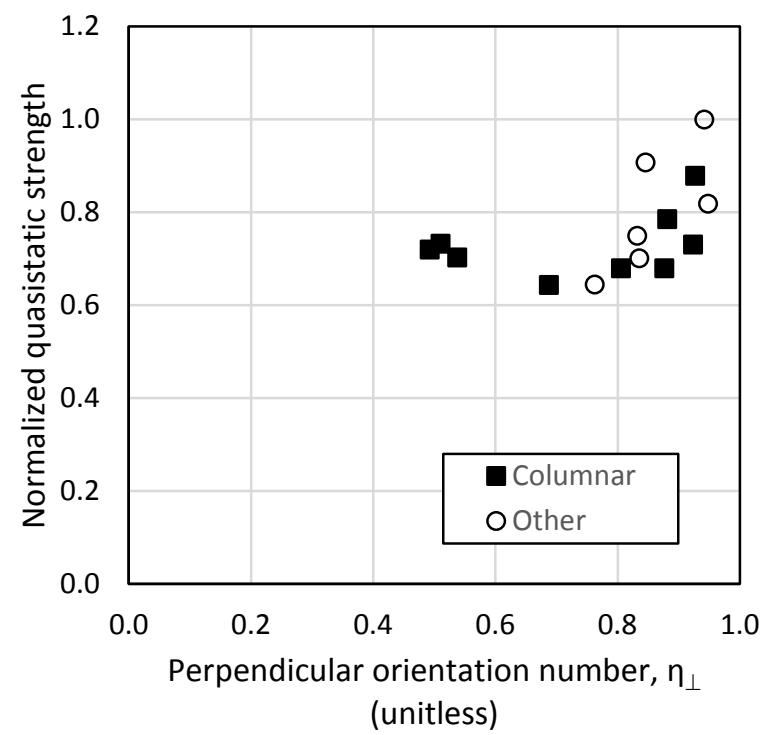

Figure 5.8. Fiber effect on quasistatic strength for different failure modes.

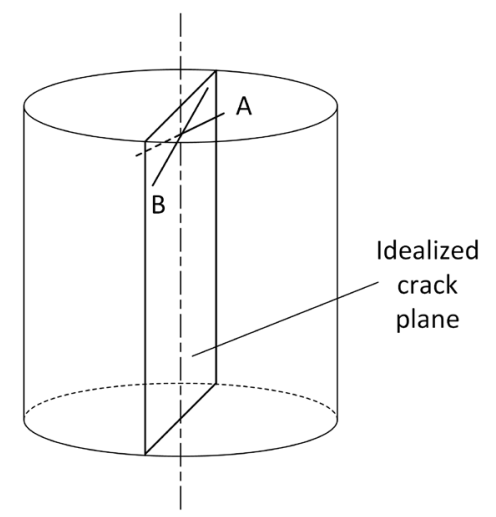

Figure 5.9. Two fibers at 45 degrees from the axis of loading. 
Note that the failure modes were influenced by the end conditions. The neoprene pads were restrained by a steel ring to reduce lateral expansion, which could induce radial stresses in the specimen. A similar phenomenon is discussed by Richardson (1991). However, the pad was not stiff enough for use at such high stresses, and deformed. Friction between the pad and the edge of the specimen likely resulted in radial tension. This served to split the specimen down its axis, as evidenced by the columnar fractures in two-thirds of the specimens. Strength results with neoprene pads were lower than expected due to the nonstandard stress distribution described above. However, the overall trend of strength versus $\eta_{\perp}$ still holds for the failure modes that occurred in the tests. Therefore, results were normalized by the maximum quasistatic compressive strength. This also highlights the relative effect of fiber orientation on strength. It must be emphasized that the results for the relationship between quasistatic strength and orientation number apply only to the loading conditions for which they were obtained.

Finally, the Games-Howell method was used to assess whether the quasistatic compressive strength was significantly different for cores taken in different directions. Recall that the cores were drilled in the $x$-, $y$-, and $z$-directions for the beam (refer to Figure 3.4 for the coordinate system). Loads were then applied along the axis of the core: $x$-direction cores were loaded along the $x$-axis, and so on. Results of this statistical test at a $95 \%$ confidence level $(\alpha=0.05)$ are shown in Table 5.4. The number of samples for each group was 6 . The mean quasistatic compressive strength was $16.01 \mathrm{ksi}$ for $x$ direction cores, $19.71 \mathrm{ksi}$ for $y$-direction cores, and $16.54 \mathrm{ksi}$ for $z$-direction cores. Remember that these cores had unusually low strengths due to the testing conditions. The differences were not significant, though the $p$-value for the $\mathrm{X}-\mathrm{Y}$ comparison was very close to the level of significance for the test. More samples would be needed to draw a definitive conclusion. However, when considered in conjunction with the plot of normalized strength versus $\eta_{\perp}$ (Figure 5.8), it seems that fiber orientation does have an effect on quasistatic compressive strength. 
Table 5.4. Results of Games-Howell comparison for quasistatic compressive strength.

\begin{tabular}{cccc}
\hline \hline Comparison & $\begin{array}{c}\text { Difference of means } \\
\text { (ksi) }\end{array}$ & $\begin{array}{c}\text { 95\% CI for difference } \\
\text { (ksi) }\end{array}$ & Adjusted $\boldsymbol{p}$-value \\
\hline $\mathrm{Y}-\mathrm{X}$ & 3.69 & $(-0.06,7.45)$ & 0.053 \\
$\mathrm{Z}-\mathrm{X}$ & 0.52 & $(-1.57,2.61)$ & 0.772 \\
$\mathrm{Z}-\mathrm{Y}$ & -3.17 & $(-6.97,0.63)$ & 0.099 \\
\hline \hline
\end{tabular}

The variation of dynamic compressive strength with orientation number is shown in Figure 5.10. Here, the strengths are normalized by the maximum dynamic compressive strength. Only 16 data points are shown; all 18 specimens were CT scanned, but the data for 2 specimens were unusable. There is considerable scatter when looking at strength versus $\eta_{\|}$or $\eta_{\perp}$. It seems that dynamic failure strength is independent of the orientation number. This is not to say that fiber orientation has no effect on failure under dynamic compression: several specimens failed along planes that had very few fibers (see Table 4.6). However, the presence of weak planes depends on the fiber orientation as well as the distribution of fibers. The existence of weak planes cannot be characterized by measures such as orientation number.

It is worth noting that the effect of orientation on strength is different at quasistatic and dynamic rates of loading. While there seems to be an increase in quasistatic strength with increasing $\eta_{\perp}$, no such trend is apparent for dynamic strength. 


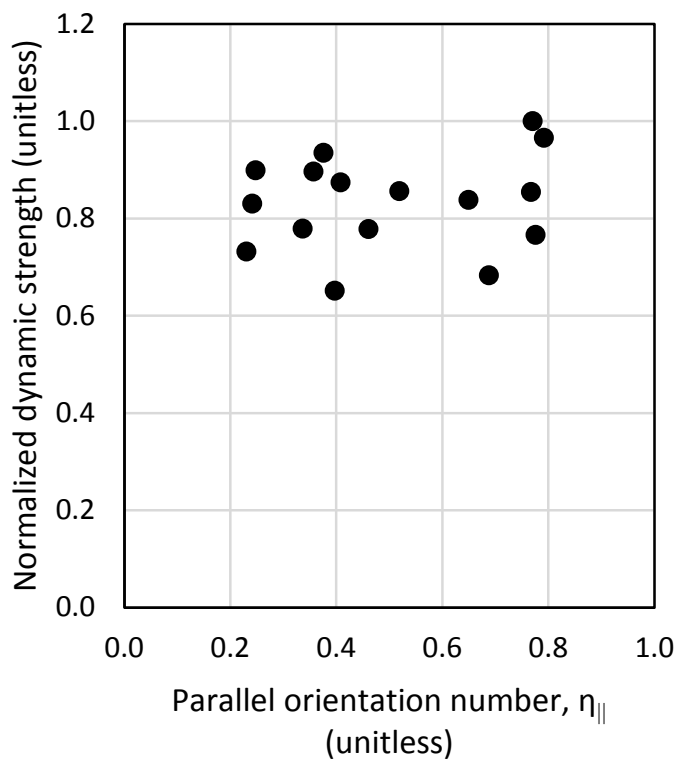

$a$.

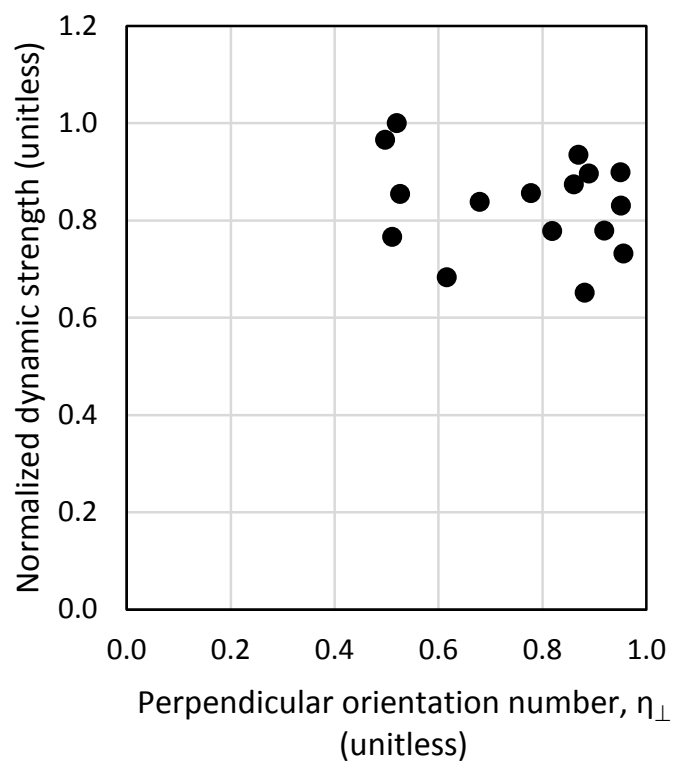

$b$.

Figure 5.10. Variation of dynamic compressive strength with $a$. parallel orientation number and $b$. perpendicular orientation number.

\subsubsection{Ductility under dynamic loading}

All 18 stress-strain curves from dynamic compression tests of Cor-Tuf are shown overlaid on one set of axes in Figure 5.11. In interpreting this data, the following caveats should be remembered. First, the measured stress-strain response is not necessarily representative of material behavior, as the specimen is not in equilibrium until very near the peak stress. Second, post-peak results may not be meaningful. Once the specimen fails, its wave propagation characteristics change; also, it may not even be in contact with the bars after failure. Nevertheless, these results are useful for comparing the overall response of specimens. The initial portions of the stress-strain curves are very similar, with the exception of X-D-6B, which remained intact during the test, and Z-D-3M. The reason for Z-D-3M's difference is not known, but may be due to the specimen not being completely seated before loading. 


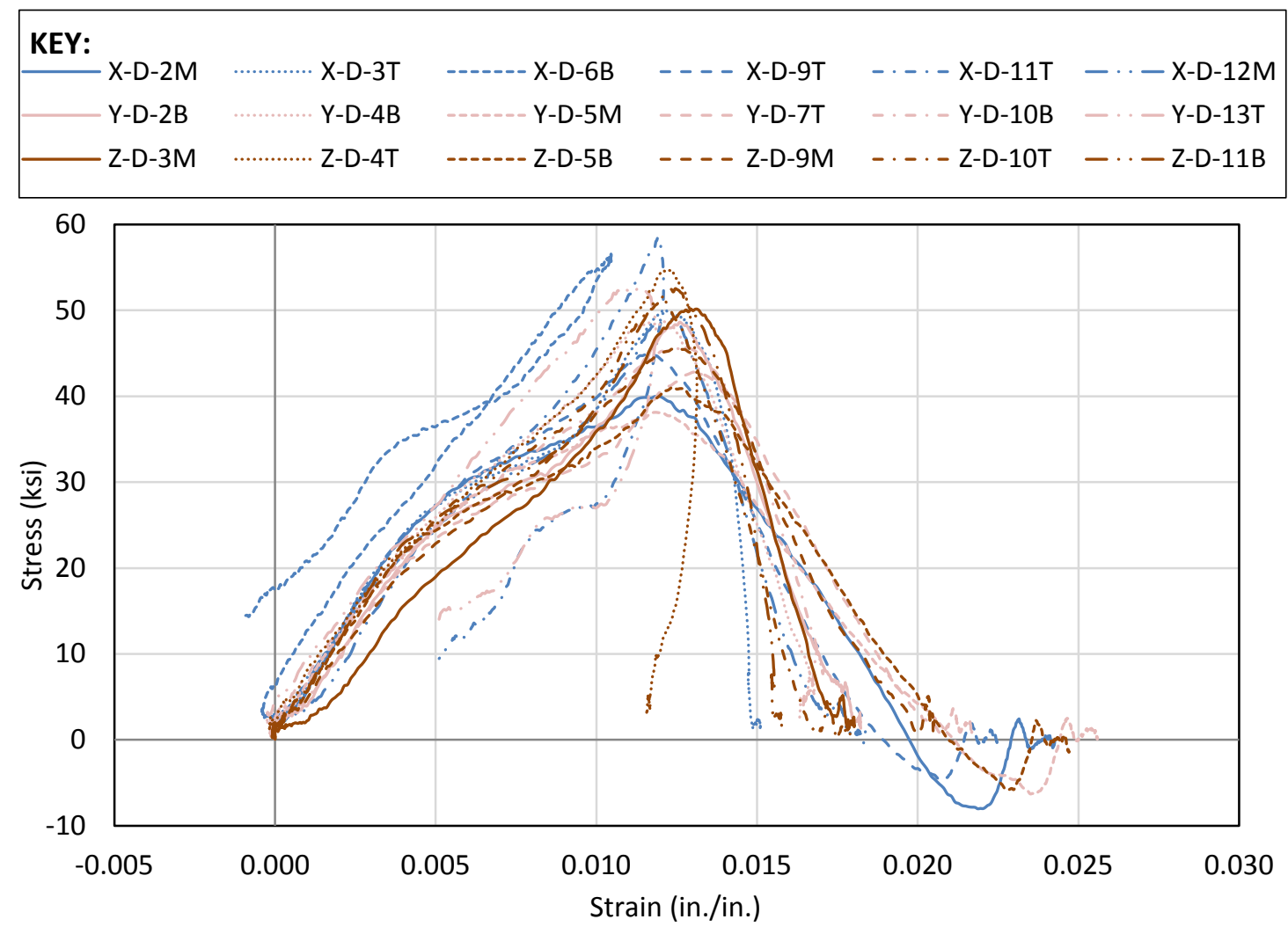

Figure 5.11. Stress-strain curves for all dynamic compression tests.

The strain at peak stress, also called the critical strain, $\varepsilon_{c r}$, is used as a measure of the ductility of the specimens. Figure 5.12 shows the effect of orientation number on the ductility (strain at peak stress), which is normalized by the maximum ductility (Table 4.6). Though there is scatter, ductility increases with $\eta_{\perp}$. When loaded by a stress wave from the input bar, the specimen expanded radially due to the Poisson effect. This expansion caused tensile stress and contributed to cracking parallel to the applied load. Hence, fibers that were perpendicular to the axis of loading were most effective in crack bridging. Therefore, by bridging cracks caused by radial expansion, the fibers kept the specimen intact longer so as to reach a higher axial strain. Best-fit lines determined by linear regression are shown in Figure 5.12. The correlation is weak, which may be due to experimental scatter. The correlation coefficients are $R^{2}=0.24$ and $R^{2}=0.27$ for $\varepsilon_{c r}$ as a function of $\eta_{\|}$and $\eta_{\perp}$, respectively. Though it is debatable whether the correlation 
with $\eta_{\perp}$ is significantly stronger, the perpendicular orientation number is a better descriptor of the physical cause for the increased ductility.

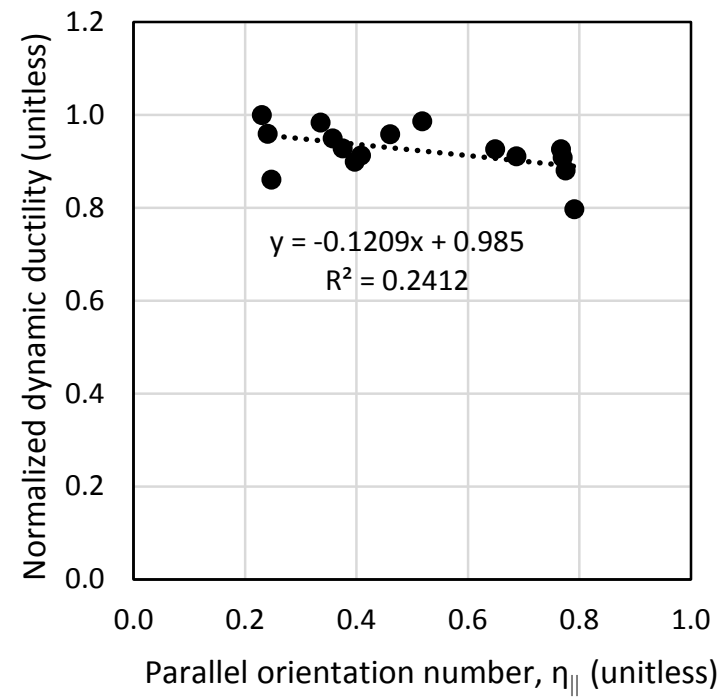

$a$.

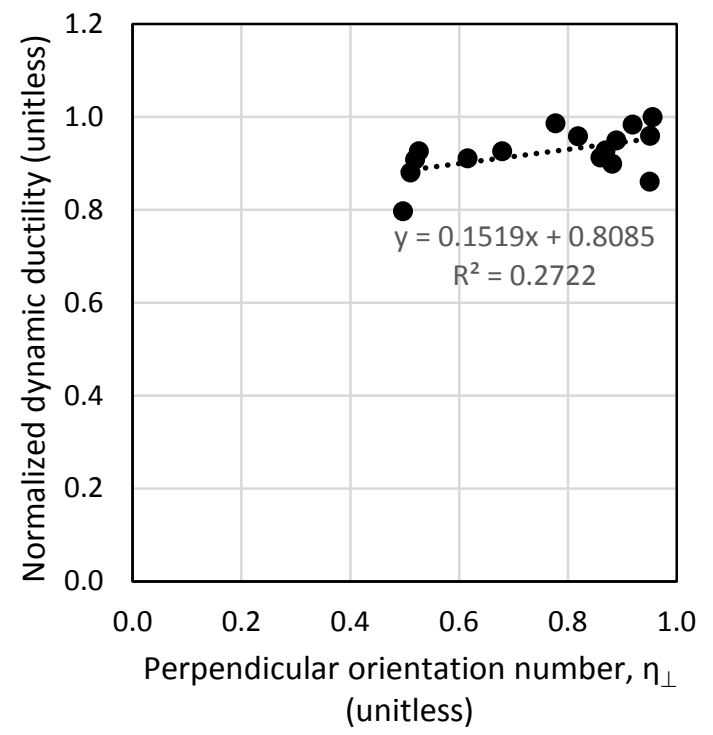

b.

Figure 5.12. Variation of ductility with $a$. parallel orientation number and $b$. perpendicular orientation number.

\subsection{Influence of Loading Rate}

The effect of loading rate on failure strength can be quantified using the DIF. Because the DIF is the ratio of dynamic strength to quasistatic strength, the choice of specimen for quasistatic strength is important. Quasistatic tests were carried out primarily with cores, so as to capture different fiber orientations. Some molded cylinders were also tested by ERDC staff for quality control purposes. DIFs are given in Table 5.5 for the data as a whole and for each core direction individually. Recall that the core directions correspond to the beam coordinate system (see Figure 3.4). The average quasistatic strength from cores tested without neoprene pads is used as a reference to calculate the DIF. In this way, the same specimen type (core) is used for both dynamic and quasistatic strengths. The number of samples indicated is the number for each set of tests: overall, 18 dynamic tests were performed. Dynamic tests achieved a maximum strain rate of 136-196 s $\mathrm{s}^{-1}$. 
Quasistatic tests were performed at a loading rate of $150 \mathrm{psi} / \mathrm{s}$; assuming an elastic modulus of $5930 \mathrm{ksi}$ (see Table 2.1), this corresponds to a strain rate of $2.5 \times 10^{-5} \mathrm{~s}^{-1}$.

Table 5.5. DIFs using core quasistatic strengths as the reference.

\begin{tabular}{ccccc}
\hline \hline \multirow{2}{*}{ Group } & \multicolumn{2}{c}{ Average failure strength (ksi) } & Avg. & DIF \\
\cline { 2 - 3 } & Dynamic & Quasistatic & DIF & COV \\
\hline Overall $(\mathrm{N}=18)$ & 48.38 & 26.62 & 1.82 & $11.7 \%$ \\
$\mathrm{X}(\mathrm{N}=6)$ & 49.84 & 26.62 & 1.87 & $14.0 \%$ \\
$\mathrm{Y}(\mathrm{N}=6)$ & 46.09 & 26.62 & 1.73 & $11.1 \%$ \\
$\mathrm{Z}(\mathrm{N}=6)$ & 49.20 & 26.62 & 1.85 & $10.2 \%$ \\
\hline \hline
\end{tabular}

The average dynamic failure strength differs little between core directions, and the average DIFs range from 1.73 to 1.87 . Overall, individual specimen DIFs range from 1.43 to 2.20. Previous tests of thermally-treated Cor-Tuf specimens ( $3 \times 3$ in. cylinders) with a cannon pressure of 80 psi have shown an average DIF of 1.96 with a COV of $11 \%$ (VanSlembrouck 2015). VanSlembrouck's overall work indicated a DIF for Cor-Tuf ranging from 1.27 to 2.09 . The overall $\mathrm{COV}$ is $11.7 \%$, which is also in line with previous results. Another UHPC, Lafarge Ductal ${ }^{\circledR}$, has exhibited higher DIFs in testing, 1.212.45 for thermally-treated specimens (Clark 2013).

The significance of core direction on the DIF (core quasistatic reference) was assessed using the Games-Howell method for simultaneous comparison in Minitab. Results of this statistical test at a 95\% confidence level $(\alpha=0.05)$ are shown in Table 5.6. The number of samples for each direction was 6. None of the DIFs are significantly different from the others. This is in agreement with the previous conclusion that the dynamic strength is independent of orientation number. Here, the core direction is a stand-in for orientation: recall that the mean orientation angle was shown to be significantly different between core directions (Table 5.1). 
Table 5.6. Results of Games-Howell comparison for DIF.

\begin{tabular}{cccc}
\hline \hline Comparison & $\begin{array}{c}\text { Difference of means } \\
\text { (unitless) }\end{array}$ & $\begin{array}{c}\text { 95\% CI for difference } \\
\text { (unitless) }\end{array}$ & Adjusted $\boldsymbol{p}$-value \\
\hline $\mathrm{Y}-\mathrm{X}$ & -0.141 & $(-0.510,0.228)$ & 0.559 \\
$\mathrm{Z}-\mathrm{X}$ & -0.024 & $(-0.391,0.343)$ & 0.982 \\
$\mathrm{Z}-\mathrm{Y}$ & 0.117 & $(-0.185,0.418)$ & 0.557 \\
\hline \hline
\end{tabular}

\subsection{Possible Sources of Error}

\subsubsection{Stress equilibrium}

To assess the validity of dynamic compression test results, stress equilibrium was determined using the definition presented in Section 2.3.7. Results of stress equilibrium analysis are given in Table 5.7. Times to equilibrium and peak stress are given relative to the start of loading, which was defined as the first positive strain rate value not followed by a fluctuation back into negative values. This was used as an unambiguous method to define the start of loading for each test. Also note that times are given based on signals from both the $\mathrm{A} / \mathrm{B}$ and $\mathrm{C} / \mathrm{D}$ channels of strain gages. It was decided not to average the channels to avoid obscuring possible discrepancies. The stress nonequilibrium at peak stress is also given.

If the specimen reaches stress equilibrium, that is, $\left|\Delta \sigma / \sigma_{m}\right| \leq 5 \%$, prior to peak stress, then the specimen failure data can be considered valid. However, an ANOVA test (Games-Howell method) indicated that attaining stress equilibrium prior to peak stress did not have a significant effect on peak stress in this data. For $\mathrm{A} / \mathrm{B}$ stress equilibrium measurements, $p=0.914$, and for $\mathrm{C} / \mathrm{D}$ stress equilibrium measurements, $p=0.089$. Remember that only two specimens did not reach stress equilibrium on the $\mathrm{C} / \mathrm{D}$ channel prior to peak stress, hence the much lower $p$-value. Finally, the level of stress nonequilibrium at peak stress (given in Table 5.7) is not necessarily a good indication of validity. If the specimen has already begun to fail, then it is not likely that the specimen will still be in equilibrium, even if it was prior to failure. The stress nonequilibrium at 
peak stress is also more variable than the time to equilibrium, as evidenced by a higher COV.

Table 5.7. Stress equilibrium during dynamic compression.

\begin{tabular}{|c|c|c|c|c|c|c|}
\hline \multirow{2}{*}{$\begin{array}{l}\text { Specimen } \\
\text { ID }\end{array}$} & \multicolumn{2}{|c|}{$\begin{array}{c}\text { Time to } \\
\text { equilibrium }(\mu s)\end{array}$} & \multicolumn{2}{|c|}{$\begin{array}{c}\text { Time to peak } \\
\text { stress }(\mu s)\end{array}$} & \multicolumn{2}{|c|}{$\begin{array}{c}\text { Stress nonequilibrium } \\
\text { at peak stress }\end{array}$} \\
\hline & $\mathbf{A} / \mathbf{B}$ & C/D & $\mathbf{A} / \mathbf{B}$ & C/D & $\mathbf{A} / \mathbf{B}$ & C/D \\
\hline X-D-2M & 121 & 117 & 131 & 121 & $12.26 \%$ & $3.78 \%$ \\
\hline Y-D-2B & 137 & 132 & 142 & 138 & $5.37 \%$ & $6.30 \%$ \\
\hline X-D-3T & 134 & 126 & 135 & 139 & $3.96 \%$ & $12.40 \%$ \\
\hline Z-D-3M & 133 & 129 & 139 & 142 & $1.42 \%$ & $12.82 \%$ \\
\hline Z-D-4T* & 141 & 131 & 139 & 137 & $8.40 \%$ & $3.98 \%$ \\
\hline Y-D-4B & 132 & 124 & 137 & 124 & $2.04 \%$ & $4.60 \%$ \\
\hline Y-D-5M* & 126 & 112 & 125 & 114 & $5.39 \%$ & $1.39 \%$ \\
\hline Z-D-5B* & 129 & 115 & 128 & 120 & $6.35 \%$ & $4.13 \%$ \\
\hline $\mathrm{X}-\mathrm{D}-6 \mathrm{~B} *$ & 146 & 144 & 143 & 136 & $8.63 \%$ & $14.56 \%$ \\
\hline Y-D-7T & 128 & 123 & 134 & 132 & $6.03 \%$ & $17.92 \%$ \\
\hline X-D-9T & 131 & 118 & 131 & 123 & $3.02 \%$ & $11.17 \%$ \\
\hline Z-D-9M & 128 & 123 & 133 & 132 & $5.47 \%$ & $16.14 \%$ \\
\hline Z-D-10T & 133 & 120 & 137 & 128 & $2.32 \%$ & $13.64 \%$ \\
\hline Y-D-10B* & 136 & 124 & 135 & 129 & $7.70 \%$ & $7.95 \%$ \\
\hline X-D-11T & 141 & 134 & 148 & 142 & $1.75 \%$ & $4.35 \%$ \\
\hline Z-D-11B & 136 & 135 & 138 & 138 & $0.34 \%$ & $2.43 \%$ \\
\hline X-D-12M & 147 & 139 & 154 & 145 & $9.99 \%$ & $5.30 \%$ \\
\hline Y-D-13T* & 138 & 137 & 128 & 130 & $17.01 \%$ & $14.20 \%$ \\
\hline Mean & 134 & 127 & 137 & 132 & $5.97 \%$ & $8.73 \%$ \\
\hline St. Dev & 7 & 9 & 7 & 9 & $4.25 \%$ & $5.31 \%$ \\
\hline $\mathrm{COV}$ & $5.1 \%$ & $7.0 \%$ & $5.3 \%$ & $6.6 \%$ & $71.1 \%$ & $60.9 \%$ \\
\hline
\end{tabular}

*Peak stress for the bolded channel occurred before equilibrium.

Before these results may be compared to theory, some properties relevant to wave propagation must be determined. The average specific weight of the Cor-Tuf SHPB specimens was $\gamma_{s}=159 \mathrm{pcf}$. Using a typical value of $E_{s}=5930 \mathrm{ksi}$ for Cor-Tuf, the elastic wave speed of the specimens is $c_{0, s}=1.31 \times 10^{4} \mathrm{ft} / \mathrm{s}$. For a specimen with a length of 3.00 in., the specimen transit time is $\tau=19.0 \mu \mathrm{s}$. The $1045 \mathrm{~S}$ steel bar on the SHPB has $\gamma_{b}=489.6 \mathrm{pcf}, E_{b}=30,380 \mathrm{ksi}$, and $c_{0, b}=1.70 \times 10^{4} \mathrm{ft} / \mathrm{s}$. If the 
specimen diameter is the same as that of the bar, the relative acoustic impedance, defined by equation (2.25), is $\beta=0.252$.

The equations developed by Yang and Shim (2005) can be used to predict the number of wave transits required for the specimen to reach equilibrium. If the loading pulse is assumed to be uniform with a rise time of $2 \tau=38.0 \mu$ s, equation (2.24) applies. Under these assumptions, stress equilibrium should occur within 4 wave transits. If the loading pulse is assumed to increase linearly until the specimen fails, equation (2.26) applies, predicting equilibrium within 8 wave transits. Taking these two cases as bounds, stress equilibrium should occur between $76 \mu$ s and $152 \mu$ s after loading. The experimental results show that the mean time to equilibrium was $134 \mu$ s $(\mathrm{COV}=5.1 \%)$ for $\mathrm{A} / \mathrm{B}$ data and $127 \mu \mathrm{s}(\mathrm{COV}=7.0 \%)$ for $\mathrm{C} / \mathrm{D}$ data. Data from both channels falls within the bounds, but the bounds are coarse due to the irregular pulse that results when a pulse shaper is used. Such a tapered pulse would be far more difficult to model analytically than the piecewise linear pulses considered by Yang and Shim (2005). Various pulse forms are illustrated schematically in Figure 5.13. The pulse-shaped waveform was measured during testing of specimen $\mathrm{X}-\mathrm{D}-2 \mathrm{M}$.

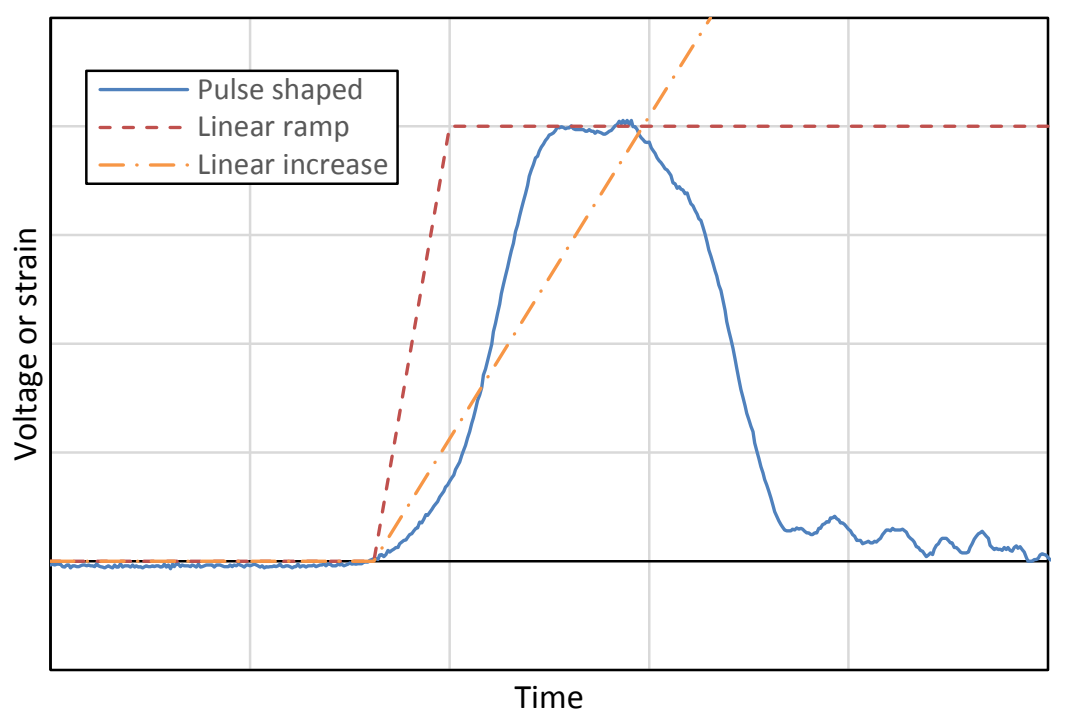

Figure 5.13. Illustration of some loading pulse forms. 


\subsubsection{Parallelness}

Only 8 of the 18 dynamic compression specimens were within the 0.5 degree parallelness tolerance when measured at Michigan Tech. This was unexpected, as these specimens received both coarse and fine grinding at ERDC. It is possible that different measurement devices at Michigan Tech and ERDC may have lead to differences in parallelness measurements. All but 2 specimens were parallel within 1 degree, and the least parallel specimen departed from parallelness by 2.01 degrees. Nonparallelness may have affected the results of dynamic compression testing: with an angled surface, stress concentrations occur on the portions of the surface that are higher, and, therefore, are loaded first.

\subsubsection{Radial confinement due to inertia}

As noted in Section 2.4, radial inertial confinement of UHPC is also a concern. Inertial loading on the specimen is proportional to the strain acceleration. A typical plot of axial strain rate and strain acceleration is given in Figure 5.14. Note the different scales and units on the two vertical axes. The measured strain rate increases to a local maximum, decreases, and then reaches a second local maximum. Strain acceleration was calculated from the strain rate using a forward difference scheme.

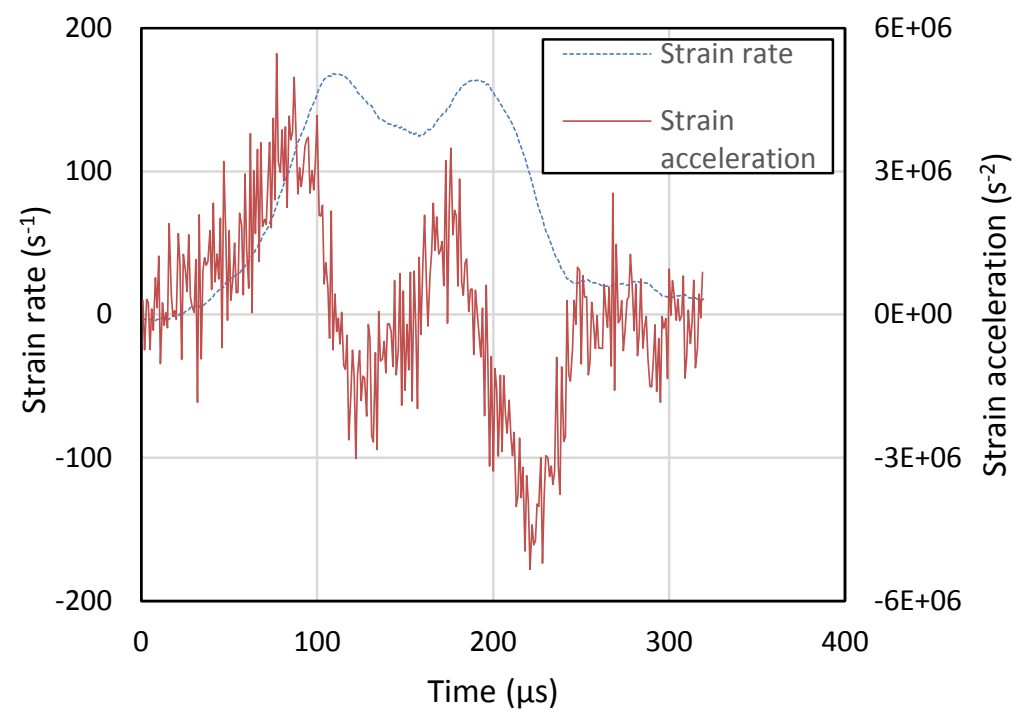

Figure 5.14. Axial strain rate and strain acceleration for specimen X-D-2M. 
Numerical differentiation amplifies any noise present in data, as the plot of strain acceleration shows. The maximum value of axial strain acceleration is of interest to determine the largest radial inertial confinement during the test. However, to obtain a reasonable estimate of radial confinement, the maximum axial strain acceleration should be minimally affected by fluctuations due to noise in the axial strain rate data. A vast selection of numerical differentiation methods are available, and a complete investigation is beyond the scope of this work. Therefore, a relatively simple least squares procedure due to Savitzky and Golay (1964; corrections by Steinier et al. 1972) was used. The Savitzky-Golay filter method is based on fitting a polynomial to the data and obtaining derivatives from the polynomial. This is done in the neighborhood of each point at which the derivative is computed, so the Savitzky-Golay filter can be considered a local method (Ahnert and Abel 2007). Implementation of the method in MATLAB was straightforward, and used a cubic least-squares fit based on seven points (see Appendix E). Strain rate and strain acceleration, determined using the Savitzky-Golay filter, are plotted in Figure 5.15. The shape of the strain acceleration is essentially preserved, but the jagged peaks are smoothed.

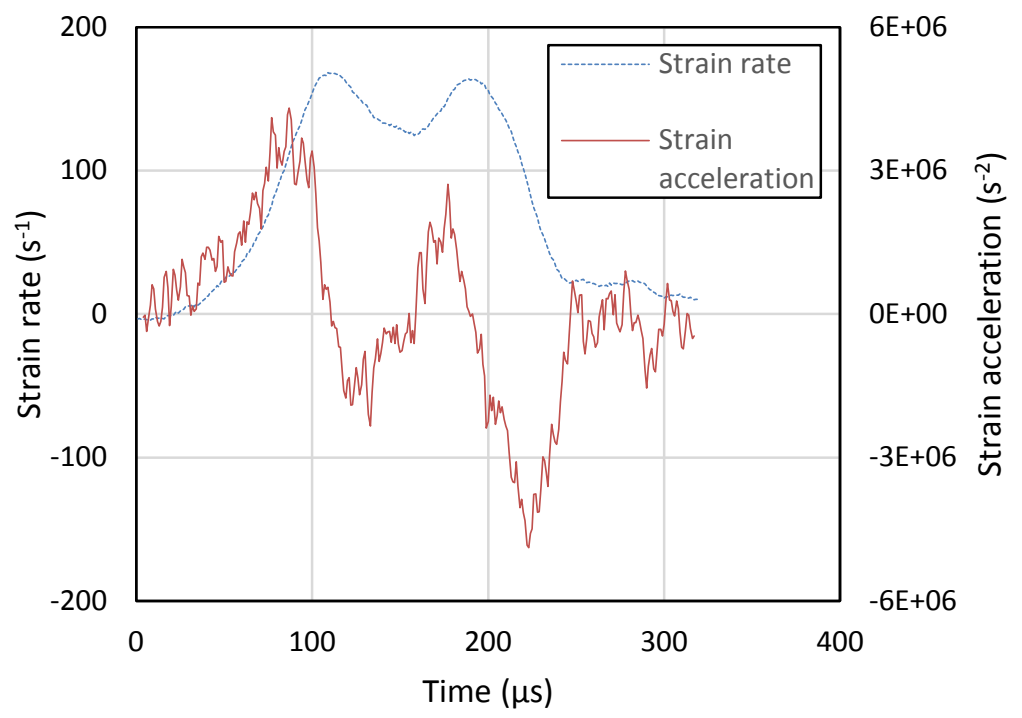

Figure 5.15. Axial strain rate and strain acceleration using a seven-point SavitzkyGolay filter for specimen X-D-2M. 
Following the analysis by Forrestal et al. (2007), confining stress due to radial inertia at the specimen's center $(r=0)$ can be calculated using equation (2.27). Table 5.8 presents the results of radial confinement calculations for the specimens. The maximum positive strain acceleration for specimens is used, as that corresponds to the maximum compressive radial stress. A typical Poisson's ratio of $v_{s}=0.23$ for Cor-Tuf (Table 2.1) is used, and the specimen diameter is assumed as $3.0 \mathrm{in}$. for the calculations. The measured specimen densities were used (see Appendix D).

The analysis assumes that Poisson's ratio is unchanged at high strain rates. Calculated confining stresses ranged from $0.17 \mathrm{ksi}$ to $0.26 \mathrm{ksi}$, with a mean of $0.22 \mathrm{ksi}$ and $\mathrm{COV}$ of $10 \%$. This level of confinement is small compared to what might be applied in a triaxial compression test, but may not be negligible compared to the tensile strength of Cor-Tuf. The quasistatic tensile strength is on the order of $1 \mathrm{ksi}$ (Table 2.1); it is not known how the tensile strength varies with loading rate.

If the forward difference method ( $1-\mu$ s time step) were used, the mean confining stress would be $0.27 \mathrm{ksi}$ with a COV of $11 \%$, and the maximum would be $0.33 \mathrm{ksi}$. The backward difference method (1- $\mu$ s time step) gives the same result. A centered difference method using the points before and after the current point (for a 2- $\mu$ s time step) results in a mean confining stress of $0.23 \mathrm{ksi}$ with a COV of $10 \%$ and maximum of $0.29 \mathrm{ksi}$. Considering the indirect nature of the calculation for radial inertial confinement, and particularly the effect of the choice of numerical differentiation method, it would be wise to consider the values in this section a rough estimate. Therefore, the maximum confining stress should probably be expressed as $0.3 \mathrm{ksi}$. A detailed study of numerical methods could shed light on which method best reduces noise while introducing the least amount of over-smoothing. Developing techniques for a less complex method of determing the inertial confining stress would also be worthwhile. 
Table 5.8. Calculated confining stress due to radial inertia.

\begin{tabular}{ccc}
\hline $\begin{array}{c}\text { Specimen } \\
\text { ID }\end{array}$ & $\begin{array}{c}\text { Max. strain } \\
\text { acceleration } \\
\left(\mathbf{1 0}^{\mathbf{6}} \mathbf{s}^{-2}\right)\end{array}$ & $\begin{array}{c}\text { Maximum } \\
\text { confinement } \\
\mathbf{( k s i )}\end{array}$ \\
\hline X-D-2M & 4.31 & 0.22 \\
X-D-3T & 4.10 & 0.21 \\
X-D-6B & 3.70 & 0.19 \\
X-D-9T & 4.06 & 0.20 \\
X-D-11T & 4.21 & 0.22 \\
X-D-12M & 3.37 & 0.17 \\
Y-D-2B & 4.17 & 0.21 \\
Y-D-4B & 4.22 & 0.21 \\
Y-D-5M & 4.47 & 0.23 \\
Y-D-7T & 4.70 & 0.24 \\
Y-D-10B & 4.26 & 0.22 \\
Y-D-13T & 4.10 & 0.21 \\
Z-D-3M & 3.77 & 0.19 \\
Z-D-4T & 4.52 & 0.23 \\
Z-D-5B & 5.26 & 0.26 \\
Z-D-9M & 4.72 & 0.24 \\
Z-D-10T & 4.63 & 0.23 \\
Z-D-11B & 4.68 & 0.24 \\
\hline Mean & 4.29 & 0.22 \\
St Dev & 0.44 & 0.02 \\
COV & $10 \%$ & $10 \%$ \\
\hline \hline
\end{tabular}

\subsubsection{Low quasistatic failure strength}

Although cored specimens are typically expected to have lower strength than molded cylinders (ASTM 2013), strength differences on the order of $10 \mathrm{ksi}$, such as those seen here, are unreasonably large. This was eventually determined to be due to the use of neoprene pads. These pads are used with lower-strength concrete specimens to distribute load more evenly; however, the pads are not suitable for use with UHPC (ASTM 2015). At a given stress level, the neoprene pads have a greater radial expansion than the UHPC specimen. This is because of neoprene's lower stiffness and high Poisson's ratio. Friction between the pads and the ends of the specimen would have resulted in radial 
tensile stresses. This is consistent with the columnar fractures seen in the majority of specimens. Expansion of the pads was restrained by a steel ring, but the pads still deformed, as evidenced by "dishing" or "cupping" of the pads after testing. The stress state induced by a deformed pad would be difficult to quantify.

This incorrect testing procedure affects only the quasistatic results. The obtained strengths are too low, but the overall trend for strength as a function of orientation number still holds for the cracking patterns in the tests. It should be emphasized that the quasistatic results, which apply to columnar and side fractures, are not necessarily what would be expected for cone or cone-and-split fractures. Dynamic results are not affected, and DIFs reported herein are based on cores tested without neoprene pads although fiber orientation data was not available for these cores. 


\section{CHAPTER 6 CONCLUSIONS AND RECOMMENDATIONS}

This thesis documents work to determine the effect of fiber orientation on the dynamic compressive behavior of Cor-Tuf. A beam was cast, and cores were taken in three directions to assess the fiber orientations occurring in a realistic structure. These cores were then tested in either quasistatic or dynamic compression. Because data on orientation and compressive behavior were collected for each specimen, correlations could be attempted. The following sections summarize the results of this work and identify possible directions for future research.

\subsection{Summary of Findings}

\subsubsection{Fiber orientation}

It was found that the fibers did align preferentially along the length of the beam, though alignment was not as pronounced as might have been expected. This alignment is likely flow-induced. Overall, the mean orientation angle with the $x$-axis (i.e., the long axis of the beam) was 39.0 degrees, with a standard deviation of 6.4 degrees. The mean orientation angle with the $y$-axis (vertical axis) was 71.2 degrees, with a standard deviation of 3.9 degrees, and the mean orientation angle with the $z$-axis (across the web thickness) was 62.2 degrees, with a standard deviation of 4.2 degrees. The differences between orientation angles for each direction were statistically significant at a $95 \%$ confidence level.

Results also showed that the mean orientation angle with the $x$-axis was less at the top and bottom of the beam than at midheight. This indicates that fibers were most aligned in the $x$-direction near the top and bottom of the form. Because no samples were taken from the outer 2 in. at the top and bottom, it is unlikely that wall effects were responsible. 


\subsubsection{Quasistatic compression}

Cored specimens tested quasistatically achieved compressive strengths of 14.3-23.6 ksi. These were lower than expected due to the use of neoprene pads. The perpendicular orientation number, $\eta_{\perp}$, for these specimens ranged from 0.493 to 0.948 . It appears that quasistatic compressive strength increases with $\eta_{\perp}$, but limited data for orientations $\eta_{\perp}<$ 0.8 make this difficult to assess. Strengthening is thought to occur by bridging of tensile cracks. These cracks form parallel to the direction of loading, hence, fibers that are perpendicular to the load are more effective. Molded cylinders used for quality control achieved quasistatic strengths of 24.6-29.7 ksi. Tests on cores without neoprene pads resulted in a mean strength of $26.6 \mathrm{ksi}$, confirming that end conditions were responsible for the low strengths in previous tests.

\subsubsection{Dynamic compression}

Cored specimens tested dynamically at strain rates of $136-196 \mathrm{~s}^{-1}$ achieved compressive strengths of 38.1-58.5 ksi. All dynamic data were calculated as the average of threewave analyses on the $\mathrm{A} / \mathrm{B}$ and $\mathrm{C} / \mathrm{D}$ strain gage channels. The value of $\eta_{\perp}$ for these specimens ranged from 0.497 to 0.956. Dynamic failure strength appears to be independent of orientation number, although results do indicate that the distribution and orientation of fibers influence failure. Three specimens (Y-D-2B, Y-D-5M, and X-D-9T) had fracture surfaces with very few fibers crossing; however, measures such as orientation number are incapable of describing the existence of fiber-free weak planes.

The mean DIF was 1.82 with a COV of $12 \%$. Note that this DIF is based on core quasistatic strengths from tests without neoprene pads. The DIF was not significantly different between core directions.

Strain at peak stress, a measure of ductility, ranged from 0.0105 to 0.0131 for specimens tested dynamically. Although strain at peak stress does increase with $\eta_{\perp}$, the correlation is fairly weak $\left(R^{2}=0.27\right)$. Fiber crack-bridging is thought to be responsible: by bridging cracks caused by radial expansion, the fibers keep the specimen intact longer so as to reach a higher axial strain. 
Stress equilibrium in the specimens was assessed separately for the $A / B$ and $C / D$ channels of output. According to A/B channel measurements, 6 specimens (out of 18) did not reach approximate equilibrium prior to peak stress. According to $\mathrm{C} / \mathrm{D}$ channel measurements, only 2 specimens did not reach approximate equilibrium. For the worst case, the difference in stress was $18 \%$ of the average stress when peak stress occurred.

At the strain rates and strain accelerations in this work, the maximum confining stress due to radial inertia was about $0.3 \mathrm{ksi}$. The information collected does not allow an assessment of the effects of this confinement, however.

\subsection{Future Work}

Although testing showed that dynamic failure strength was essentially independent of orientation number, the failure of several specimens suggest that a more detailed characterization of fiber distribution and orientation might provide insight into the failure process. One possibility is to examine the 3-D distribution of fibers to determine weak planes, either by minimizing the number of fibers crossing the plane, or by minimizing the total fiber length normal to the plane. It is also worth investigating the role of voids in initiating failure in relation to the weak planes described above.

The 3-D specimen volume data created in this work could also be used for simulations. Finite element analysis might provide a better vehicle for developing analytical models than the simple relationships examined in the current work.

The role of confinement in SHPB tests continues to be of interest. Inertial confinement can be described analytically, but its effects on strength measurements are harder to quantify. The loading produced during SHPB testing is certainly not purely uniaxial compression. More research is needed to determine the most appropriate way to interpret strength measurements from dynamic tests.

Finally, it seems appropriate to develop failure mode descriptions specific to UHPCs with fiber reinforcement. Crack-bridging by fibers leads to different fracture patterns than those seen in NSC. These descriptions might be developed for quasistatic compression failures first, and then extended to dynamic testing if required. 



\section{CHAPTER 7 REFERENCES}

ACI 239. Spring 2015 Agenda ACI 239 - Kansas City [Internet]. 2015. American Concrete Institute. [updated Nov. 13, 2015, cited May 17, 2016]. Available from: https://www.concrete.org/committees/getagendaminutes.aspx?DocID=85406

Advani SG, Tucker III CL. 1987. The use of tensors to describe and predict fiber orientation in short fiber composites. Journal of Rheology (1978-present) 31(8):751-784.

AFGC. 2013. Ultra High Performance Fibre-Reinforced Concretes: Recommendations. Paris: Association Française de Génie Civil.

Ahnert K, Abel M. 2007. Numerical differentiation of experimental data: local versus global methods. Computer Physics Communications 177(10):764-774.

ASTM. 2013. ASTM C42/C42M, Standard Test Method for Obtaining and Testing Drilled Cores and Sawed Beams of Concrete. West Conshohocken, PA: ASTM International.

ASTM. 2015. ASTM C39/C39M, Standard Test Method for Compressive Strength of Cylindrical Concrete Specimens. West Conshohocken, PA: ASTM International.

Barnett SJ, Lataste J-F, Parry T, Millard SG, Soutsos MN. 2010. Assessment of fibre orientation in ultra high performance fibre reinforced concrete and its effect on flexural strength. Materials and Structures 43(7):1009-1023.

Bertholf LD, Karnes CH. 1975. Two-dimensional analysis of the split Hopkinson pressure bar system. Journal of the Mechanics and Physics of Solids 23(1):1-19.

Bischoff PH, Perry SH. 1991. Compressive behaviour of concrete at high strain rates. Materials and Structures 24(6):425-450.

Buzug TM. 2008. Computed Tomography: From Photon Statistics to Modern Cone-Beam CT. Berlin: Springer.

Cavill B, Rebentrost M, Perry V. 2006. Ductal ${ }^{\circledR}$-An Ultra-High Performance Material for Resistance to Blasts and Impacts. 1st Specialty Conference on Disaster Mitigation; 2006; Calgary, Alberta, Canada.

Chen W, Subhash G, Ravichandran G. 1994. Evaluation of ceramic specimen geometries used in a split Hopkinson pressure bar. Dymat Journal 1(3):193-210.

Chen W, Song B. 2011. Split Hopkinson (Kolsky) Bar: Design, Testing and Applications. New York: Springer.

Chree C. 1889. The Equations of an Isotropic Elastic Solid in Polar and Cylindrical Coordinates, their Solution and Application. Transactions of the Cambridge Philosophical Society 14:250-369.

Clark JF. 2013. Preliminary Investigation of Ultra-High Performance Concrete Behavior at High Strain Rates using the Split-Hopkinson Pressure Bar [Master's thesis]. Michigan Technological University.

Davies EDH, Hunter SC. 1963. The dynamic compression testing of solids by the method of the split Hopkinson pressure bar. Journal of the Mechanics and Physics of Solids 11(3):155-179. 
de Wolski S. 2011. shrinkWrap. MATLAB program. Available from: http://www.mathworks.com/matlabcentral/fileexchange/29175-shrinkwrap.

Delsol S, Charron J-P. 2013. Numerical Modeling of UHPFRC Mechanical Behavior Based on Fibre Orientation. In: Toutlemonde F, Resplendino J, editors. Proceedings of the RILEM-fib-AFGC International Symposium on Ultra-High Performance Fiber-Reinforced Concrete; 2013; Marseille, France: RILEM Publications S.A.R.L. p. 679-688.

Drucker DC, Prager W. 1952. Soil mechanics and plastic analysis or limit design. Quarterly of Applied Mathematics 10(2):157-165.

Edgington J, Hannant DJ. 1972. Steel fibre reinforced concrete. The effect on fibre orientation of compaction by vibration. Matériaux et Construction 5(1):41-44.

Eik M, Lõhmus K, Tigasson M, Listak M, Puttonen J, Herrmann H. 2013. DCconductivity testing combined with photometry for measuring fibre orientations in SFRC. Journal of Materials Science 48(10):3745-3759.

Empelmann M, Teutsch M, Steven G. 2008. Improvement of the Post Fracture Behaviour of UHPC by Fibres. In: Fehling E, Schmidt M, Stürwald S, editors. Proceedings of the Second International Symposium on UHPC, Kassel, Germany; 2008; Kassel, Germany. p. 177-184.

Field J, Walley S, Proud W, Goldrein H, Siviour C. 2004. Review of experimental techniques for high rate deformation and shock studies. International Journal of Impact Engineering 30(7):725-775.

Flanders LF. 2014. LSFfiberOrient. MATLAB program. [In Oesch (2015)].

Follansbee PS, Frantz C. 1983. Wave propagation in the split Hopkinson pressure bar. Journal of Engineering Materials and Technology 105(1):61-66.

Forrestal MJ, Wright TW, Chen W. 2007. The effect of radial inertia on brittle samples during the split Hopkinson pressure bar test. International Journal of Impact Engineering 34(3):405-411.

Frettlöhr B. 2013. Theoretical Model for Size and Shape Effect of UHPFRC in Flexural Tension Considering Tensile Behaviour Influenced by Fibre Orientation. In: Toutlemonde F, Resplendino J, editors. Proceedings of the RILEM-fib-AFGC International Symposium on Ultra-High Performance Fiber-Reinforced Concrete; 2013; Marseille, France: RILEM Publications S.A.R.L. p. 689-698.

Frew DJ, Forrestal MJ, Chen W. 2001. A split Hopkinson pressure bar technique to determine compressive stress-strain data for rock materials. Experimental Mechanics 41(1):40-46.

Frew DJ, Forrestal MJ, Chen W. 2002. Pulse shaping techniques for testing brittle materials with a split Hopkinson pressure bar. Experimental Mechanics 42(1):93106.

Gama BA, Lopatnikov SL, Gillespie JW. 2004. Hopkinson bar experimental technique: A critical review. Applied Mechanics Reviews 57(4):223-250.

Gettu R, Gardner DR, Saldivar H, Barragán BE. 2005. Study of the distribution and orientation of fibers in SFRC specimens. Materials and Structures 38(1):31-37.

Gilbertson CG. 2011. Dynamic properties of wood using the split-Hopkinson pressure bar [PhD diss.]. Michigan Technological University. 
Goldman LW. 2007. Principles of CT and CT technology. Journal of Nuclear Medicine Technology 35(3):115-128.

Gray III GT. 2000. Classic Split-Hopkinson Pressure Bar Testing. ASM Handbook

Volume 8, Mechanical Testing and Evaluation. Materials Park, OH: ASM International. p. 462-476.

Graybeal BA. 2006. Report FHWA-HRT-06-103. Material Property Characterization of Ultra-High Performance Concrete. McLean, VA: Federal Highway Administration.

Graybeal BA. 2011. Tech Note FHWA-HRT-11-038. Ultra-high performance concrete. McLean, VA: Federal Highway Administration.

Hopkinson B. 1914. A Method of Measuring the Pressure Produced in the Detonation of High Explosives or by the Impact of Bullets. Philosophical Transactions of the Royal Society of London A 213:437-456.

Jackson JW, Waxman M. 1963. An analysis of pressure and stress distribution under rigid Bridgman-type anvils. High Pressure Measurements. Butterworth Inc. p. 39-58.

Jerome E. 1991. Analysis of a Proposed Six Inch Diameter Split Hopkinson Pressure Bar [PhD diss.]. University of Florida.

Jiao C, Sun W, Huan S, Jiang G. 2009. Behavior of steel fiber-reinforced high-strength concrete at medium strain rate. Frontiers of Architecture and Civil Engineering in China 3(2):131-136.

Ju Y, Liu H, Sheng G, Wang H. 2010. Experimental study of dynamic mechanical properties of reactive powder concrete under high-strain-rate impacts. Science China Technological Sciences 53(9):2435-2449.

Kameswara Rao CVS. 1979. Effectiveness of random fibres in composites. Cement and Concrete Research 9(6):685-693.

Kanakubo T, Miyaguchi M, Asano K. 2016. Influence of Fiber Orientation on Bridging Performance of Polyvinyl Alcohol Fiber-Reinforced Cementitious Composite. ACI Materials Journal 113(2):131-141.

Kang S-T, Kim J-K. 2011. The relation between fiber orientation and tensile behavior in an Ultra High Performance Fiber Reinforced Cementitious Composites (UHPFRCC). Cement and Concrete Research 41(10):1001-1014.

Karhunen K, Seppänen A, Lehikoinen A, Monteiro PJM, Kaipio JP. 2010. Electrical resistance tomography imaging of concrete. Cement and Concrete Research 40(1):137-145.

Kim SW, Kang ST, Park JJ, Ryu GS. 2008. Effect of Filling Method on Fibre Orientation $\&$ Dispersion and Mechanical Properties of UHPC. In: Fehling E, Schmidt M, Stürwald S, editors. Proceedings of the Second International Symposium on UHPC, Kassel, Germany; 2008; Kassel, Germany. p. 185-192.

Kolsky H. 1949. An investigation of the mechanical properties of materials at very high rates of loading. Proceedings of the Physical Society B 62(11):676-700.

Krafft JM, Sullivan AM, Tipper CF. 1954. The effect of static and dynamic loading and temperature on the yield stress of iron and mild steel in compression. Proceedings of the Royal Society of London A 221(1144):114-127. 
Krause M, Hausherr JM, Burgeth B, Herrmann C, Krenkel W. 2010. Determination of the fibre orientation in composites using the structure tensor and local X-ray transform. Journal of Materials Science 45(4):888-896.

Krenchel H. 1964. Fibre reinforcement: theoretical and practical investigations of the elasticity and strength of fibre-reinforced materials [PhD diss.]. Technical University of Denmark.

Krenchel H. 1975. Fibre spacing and specific fibre surface. In: Neville A, editor. Fibre Reinforced Cement and Concrete. UK: The Construction Press. p. 69-79.

Lafarge North America. Ductal ${ }^{\circledR}$ Reference T 009. Operating Procedure Cylinder End Preparation. Calgary, Alberta, Canada.

Lai J, Sun W. 2009. Dynamic behaviour and visco-elastic damage model of ultra-high performance cementitious composite. Cement and Concrete Research 39(11):1044-1051.

Landis EN, Keane DT. 2010. X-ray microtomography. Materials Characterization 61(12):1305-1316.

Laranjeira F, Aguado A, Molins C, Grünewald S, Walraven J, Cavalaro S. 2012. Framework to predict the orientation of fibers in FRC: a novel philosophy. Cement and Concrete Research 42(6):752-768.

Lee Y, Lee S, Youn J, Chung K, Kang T. 2002. Characterization of fiber orientation in short fiber reinforced composites with an image processing technique. Materials Research Innovations 6(2):65-72.

Leonhardt S, Lowke D, Gehlen C. 2012. Effect of Fibres on Impact Resistance of Ultra High Performance Concrete. In: Schmidt M, Fehling E, Glotzbach C, Fröhlich S, Piotrowski S, editors. Proceedings of the 3rd International Symposium on UHPC and Nanotechnology for High Performance Construction Materials; 2012; Kassel, Germany. p. 811-817.

Li QM, Meng H. 2003. About the dynamic strength enhancement of concrete-like materials in a split Hopkinson pressure bar test. International Journal of Solids and Structures 40(2):343-360.

Li VC, Wang Y, Backer S. 1991. A micromechanical model of tension-softening and bridging toughening of short random fiber reinforced brittle matrix composites. Journal of the Mechanics and Physics of Solids 39(5):607-625.

Li VC. 1992. A simplified micromechanical model of compressive strength of fiberreinforced cementitious composites. Cement and Concrete Composites 14(2):131141.

Lindholm US, Yeakley LM. 1968. High strain-rate testing: tension and compression. Experimental Mechanics 8(1):1-9.

Mansur MA, Chin MS, Wee TH. 1999. Stress-strain relationship of high-strength fiber concrete in compression. Journal of Materials in Civil Engineering 11(1):21-29.

Martinie L, Roussel N. 2011. Simple tools for fiber orientation prediction in industrial practice. Cement and Concrete Research 41(10):993-1000.

Martz HE, Scheberk DJ, Roberson GP, Monteiro PJM. 1993. Computerized tomography analysis of reinforced concrete. ACI Materials Journal 90(3):259-264. 
Millard SG, Molyneaux TCK, Barnett SJ, Gao X. 2010. Dynamic enhancement of blastresistant ultra high performance fibre-reinforced concrete under flexural and shear loading. International Journal of Impact Engineering 37(4):405-413.

Minitab. 2010. Minitab 17 Statistical Software. State College, PA. Computer program.

Molins C, Aguado A, Saludes S. 2009. Double punch test to control the energy dissipation in tension of FRC (Barcelona test). Materials and Structures 42(4):415-425.

Mondal AB. 2012. Dynamic Triaxial Compression Experiments on Cor-Tuf Specimens [Master's thesis]. Purdue University.

Moser RD, Allison PG, Chandler MQ. 2013. Report ERDC/GSL TR-13-17. Spatiallyresolved characterization techniques to investigate impact damage in ultra-high performance concretes. Vicksburg, MS: U.S. Army Engineer Research and Development Center.

Oesch TS. 2014a. FAnalysis. MATLAB program. [In Oesch (2015)].

Oesch TS. 2014b. FCorrect. MATLAB program. [In Oesch (2015)].

Oesch TS. 2014c. FStatistics. MATLAB program. [In Oesch (2015)].

Oesch TS. 2014d. IAccumulator. MATLAB program. [In Oesch (2015)].

Oesch TS. 2014e. ICorrect. MATLAB program. [In Oesch (2015)].

Oesch TS. 2014f. LSFfiberOrient. MATLAB program. [In Oesch (2015)].

Oesch TS. 2014g. shrinkWrap. MATLAB program. [In Oesch (2015)].

Oesch TS. 2015. Investigation of fiber and cracking behavior for conventional and ultrahigh performance concretes using $\mathrm{x}$-ray computed tomography [PhD diss.]. University of Illinois at Urbana-Champaign.

Othman H, Marzouk H. 2016. Strain Rate Sensitivity of Fiber-Reinforced Cementitious Composites. ACI Materials Journal 113(2):143-150.

Pansuk W, Sato H, Sato Y, Shionaga R. 2008. Tensile Behaviors and Fiber Orientation of UHPC. In: Fehling E, Schmidt M, Stürwald S, editors. Proceedings of the Second International Symposium on UHPC, Kassel, Germany; 2008; Kassel, Germany. p. $161-168$

Pochhammer L. 1876. Ueber die Fortpflanzungsgeschwindigkeiten kleiner Schwingungen in einem unbegrenzten isotropen Kreiscylinder. [On the propagation velocities of small oscillations in an unlimited isotropic circular cylinder.]. Journal für die reine und angewandte Mathematik 81:324-336.

Radon J. 1917. Über die Bestimmung von Funktionen durch ihre Integralwerte längs gewisser Mannigfal-ligkeiten. Ber. Verh. Sächs. Akad. Wiss. Leipzig Math. Phys. Kl. 69:262-277.

Radon J. 1986. On the determination of functions from their integral values along certain manifolds. IEEE Transactions on Medical Imaging 5(4):170-176.

Rand JL. 1967. Report NOLTR 67-156. An Analysis of the Split Hopkinson Pressure Bar. White Oak, MD: U.S. Naval Ordnance Laboratory.

Ravichandran G, Subhash G. 1994. Critical appraisal of limiting strain rates for compression testing of ceramics in a split Hopkinson pressure bar. Journal of the American Ceramic Society 77(1):263-267.

Richard P, Cheyrezy M. 1995. Composition of reactive powder concretes. Cement and Concrete Research 25(7):1501-1511. 
Richardson DN. 1991. Review of variables that influence measured concrete compressive strength. Journal of Materials in Civil Engineering 3(2):95-112.

Rong Z, Sun W, Zhang Y. 2010. Dynamic compression behavior of ultra-high performance cement based composites. International Journal of Impact Engineering 37(5):515-520.

Ross CA, Tedesco JW, Kuennen ST. 1995. Effects of strain rate on concrete strength. ACI Materials Journal 92(1):37-45.

Roth MJ, Rushing TS, Flores OG, Sham DK, Stevens JW. 2010. Report ERDC/GSL TR10-46. Laboratory Investigation of the Characterization of Cor-Tuf Flexural and Splitting Tensile Properties. Vicksburg, MS: U.S. Army Engineer Research and Development Center.

Savitzky A, Golay MJ. 1964. Smoothing and Differentiation of Data by Simplified Least Squares Procedures. Analytical Chemistry 36(8):1627-1639.

Schneider CA, Rasband WS, Eliceiri KW. 2012. NIH Image to ImageJ: 25 years of image analysis. Nature Methods 9(7):671-675.

Schnell J, Ackermann FP, Rösch R, Sych T. 2008. Statistical analysis of the fibre distribution in ultra high performance concrete using computer tomography. In: Fehling E, Schmidt M, Stürwald S, editors. Proceedings of the Second International Symposium on UHPC, Kassel, Germany; 2008; Kassel, Germany. p. 145-152.

Scott DA, Long WR, Moser RD, Green BH, O'Daniel JL, Williams BA. 2015. Report ERDC/GSL TR-15-22. Impact of Steel Fiber Size and Shape on the Mechanical Properties of Ultra-High Performance Concrete. Vicksburg, MS: U.S. Army Engineer Research and Development Center.

Simon A, Corvez D, Marchand P. 2013. Feedback of a Ten Years Assessment of Fibre Distribution using K Factor Concept. In: Toutlemonde F, Resplendino J, editors. Proceedings of the RILEM-fib-AFGC International Symposium on Ultra-High Performance Fiber-Reinforced Concrete; 2013; Marseille, France: RILEM Publications S.A.R.L. p. 669-678.

Steinier J, Termonia Y, Deltour J. 1972. Comments on Smoothing and Differentiation of Data by Simplified Least Square Procedure. Analytical Chemistry 44(11):19061909.

Stroeven P. 1978. Morphometry of fibre reinforced cementitious materials. Part I : Efficiency and spacing in idealized structures. Matériaux et Construction 11(1):31-38.

Stroeven P. 1979. Morphometry of fibre reinforced cementitious materials. Part II: Inhomogeneity, segregation and anisometry of partially oriented fibre structures. Matériaux et Construction 12(1):9-20.

Stroeven P. 2009. Stereological principles of spatial modeling applied to steel fiberreinforced concrete in tension. ACI Materials Journal 106(3):213-222.

Su Y-M. 2012. Characterization of concrete containing reclaimed asphalt pavement by superpave indirect tensile test and X-ray computed tomography [PhD diss.]. University of Florida.

Su Y, Li J, Wu C, Wu P, Li Z-X. 2016. Effects of steel fibres on dynamic strength of UHPC. Construction and Building Materials 114:708-718. 
Swamy RN. 1975. Fibre reinforcement of cement and concrete. Matériaux et Construction 8(3):235-254.

The MathWorks. 2014a. Image Processing Toolbox (version 9.0). Natick, MA. MATLAB toolbox.

The MathWorks. 2014b. MATLAB (version R2014a). Natick, MA. Computer program.

Trainor KJ, Flanders LF, Landis EN. 2013. 3D measurements to determine micromechanical energy dissipation in steel fiber reinforced concrete. In: van Mier JGM, Ruiz G, Andrade C, Yu RC, Zhang XX, editors. VIII International Conference on Fracture Mechanics of Concrete and Concrete Structures; 2013 2013a; Toledo, Spain.

Trainor KJ, Foust BW, Landis EN. 2013b. Measurement of energy dissipation mechanisms in fracture of fiber-reinforced ultrahigh-strength cement-based composites. Journal of Engineering Mechanics 139(7):771-779.

University of Maine. 2013. NSIExtractor. Orono, ME. MATLAB program. [In Oesch (2015)].

VanSlembrouck DJ. 2015. Compression behavior at high strain rate for an ultra high performance concrete [Master's report]. Michigan Technological University.

Williams EM, Graham SS, Reed PA, Rushing TS. 2009. Report ERDC/GSL TR-09-22. Laboratory Characterization of Cor-Tuf Concrete With and Without Steel Fibers. Vicksburg, MS: U.S. Army Engineer Research and Development Center.

Woo LY, Wansom S, Ozyurt N, Mu B, Shah SP, Mason TO. 2005. Characterizing fiber dispersion in cement composites using AC-Impedance Spectroscopy. Cement and Concrete Composites 27(6):627-636.

Yang L, Shim V. 2005. An analysis of stress uniformity in split Hopkinson bar test specimens. International Journal of Impact Engineering 31(2):129-150.

Young IT, Gerbrands JJ, van Vliet LJ. 1998. Fundamentals of image processing. Delft, The Netherlands: Delft University of Technology.

Zhang M, Wu H, Li Q, Huang F. 2009. Further investigation on the dynamic compressive strength enhancement of concrete-like materials based on split Hopkinson pressure bar tests. Part I: Experiments. International Journal of Impact Engineering 36(12):1327-1334.

Zollo RF. 1997. Fiber-reinforced concrete: an overview after 30 years of development. Cement and Concrete Composites 19(2):107-122. 



\section{APPENDIX A End Parallelness Measurements}

Note that parallelness measurements on quasistatic compression test specimens (Table A.1 and Table A.2) were made at ERDC, and measurements on dynamic compression test specimens (Table A.3) were made at Michigan Tech.

Table A.1. Quasistatic compression test specimen parallelness measurements.

\begin{tabular}{lccccccccc}
\hline \hline & \multicolumn{3}{c}{ Height measurement (in.) } & $\begin{array}{c}\text { Average } \\
\text { diameter }\end{array}$ & $\begin{array}{c}\text { Allowable } \\
\text { deviation }\end{array}$ & $\begin{array}{c}\text { Max } \\
\text { absolute } \\
\text { deviation } \\
\text { (in.) }\end{array}$ & $\begin{array}{c}\text { Deviation } \\
\text { from } \\
\text { parallelness } \\
\text { (degrees) }\end{array}$ \\
\cline { 2 - 11 } & $\mathbf{1}$ & $\mathbf{2}$ & $\mathbf{3}$ & $\mathbf{4}$ & Center & (in.) & 0.30 \\
\hline 3B & 0.0170 & 0.0243 & 0.0157 & 0.0089 & 0.0165 & 3.0240 & 0.0132 & 0.0078 & 0.013 \\
5T & 0.0212 & 0.0275 & 0.0166 & 0.0179 & 0.0232 & 3.0165 & 0.0132 & 0.0066 & 0.25 \\
6M & 0.0141 & 0.0105 & 0.0150 & 0.0148 & 0.0121 & 3.0280 & 0.0132 & 0.0029 & 0.11 \\
7M & 0.0179 & 0.0206 & 0.0244 & 0.0215 & 0.0210 & 3.0172 & 0.0132 & 0.0034 & 0.13 \\
7B & 0.0153 & 0.0142 & 0.0013 & 0.0010 & 0.0064 & 3.0158 & 0.0132 & 0.0089 & 0.34 \\
8T & 0.0235 & 0.0225 & 0.0125 & 0.0141 & 0.0163 & 3.0160 & 0.0132 & 0.0072 & 0.27 \\
8M & 0.0041 & 0.0054 & 0.0082 & 0.0048 & 0.0048 & 3.0257 & 0.0132 & 0.0034 & 0.13 \\
8B & 0.0241 & 0.0182 & 0.0341 & 0.0400 & 0.0298 & 3.0207 & 0.0132 & 0.0116 & 0.44 \\
9B & 0.0035 & 0.0037 & 0.0064 & 0.0068 & 0.0051 & 3.0280 & 0.0132 & 0.0017 & 0.06 \\
10M & 0.0264 & 0.0186 & 0.0246 & 0.0286 & 0.0260 & 3.0227 & 0.0132 & 0.0074 & 0.28 \\
11M & 0.0190 & 0.0114 & 0.0215 & 0.0145 & 0.0135 & 3.0237 & 0.0132 & 0.0080 & 0.30 \\
12T & 0.0193 & 0.0214 & 0.0222 & 0.0210 & 0.0216 & 3.0192 & 0.0132 & 0.0023 & 0.09 \\
12B & 0.0001 & 0.0004 & 0.0003 & 0.0011 & 0.0004 & 3.0223 & 0.0132 & 0.0007 & 0.03 \\
13M & 0.0120 & 0.0191 & 0.0348 & 0.0250 & 0.0226 & 3.0245 & 0.0132 & 0.0122 & 0.46 \\
13B & 0.0233 & 0.0177 & 0.0117 & 0.0159 & 0.0170 & 3.0278 & 0.0132 & 0.0063 & 0.24 \\
14T & 0.0127 & 0.0004 & 0.0097 & 0.0098 & 0.0053 & 3.0163 & 0.0132 & 0.0074 & 0.28 \\
14M & 0.0060 & 0.0098 & 0.0050 & 0.0011 & 0.0053 & 3.0282 & 0.0132 & 0.0045 & 0.17 \\
15T & 0.0132 & 0.0169 & 0.0034 & 0.0054 & 0.0096 & 3.0278 & 0.0132 & 0.0073 & 0.28 \\
\hline \hline
\end{tabular}

*For the sake of brevity, only the specimen position is given. 
Table A.2. Additional quasistatic compression test specimen parallelness measurements.

\begin{tabular}{|c|c|c|c|c|c|c|c|c|c|}
\hline \multirow{2}{*}{ ID* } & \multicolumn{5}{|c|}{ Height measurement (in.) } & \multirow{2}{*}{$\begin{array}{c}\text { Average } \\
\text { diameter } \\
\text { (in.) }\end{array}$} & \multirow{2}{*}{$\begin{array}{c}\text { Allowable } \\
\text { deviation } \\
\text { (in.) }\end{array}$} & \multirow{2}{*}{$\begin{array}{c}\text { Max } \\
\text { absolute } \\
\text { deviation } \\
\text { (in.) }\end{array}$} & \multirow{2}{*}{$\begin{array}{l}\text { Deviation } \\
\text { from } \\
\text { parallelness } \\
\text { (degrees) }\end{array}$} \\
\hline & 1 & 2 & 3 & 4 & Center & & & & \\
\hline $1 \mathrm{~T}$ & 0.0095 & 0.0111 & 0.0117 & 0.0113 & 0.0105 & 3.023 & 0.0132 & 0.0012 & 0.05 \\
\hline $1 \mathrm{~B}$ & 0.0244 & 0.0191 & 0.0221 & 0.0250 & 0.0219 & 3.009 & 0.0131 & 0.0031 & 0.12 \\
\hline $2 \mathrm{~T}$ & 0.0201 & 0.0234 & 0.0200 & 0.0138 & 0.0200 & 3.010 & 0.0131 & 0.0062 & 0.24 \\
\hline $15 \mathrm{~B}$ & 0.0220 & 0.0162 & 0.0160 & 0.0322 & 0.0239 & 3.023 & 0.0132 & 0.0083 & 0.31 \\
\hline $16 \mathrm{~T}$ & 0.0071 & 0.0226 & 0.0291 & 0.0162 & 0.0186 & 3.022 & 0.0132 & 0.0115 & 0.44 \\
\hline $16 \mathrm{M}$ & 0.0146 & 0.0062 & 0.0158 & 0.0250 & 0.0153 & 3.020 & 0.0132 & 0.0097 & 0.37 \\
\hline
\end{tabular}

*For the sake of brevity, only the specimen position is given.

Table A.3. Dynamic compression test specimen parallelness measurements.

\begin{tabular}{|c|c|c|c|c|c|c|c|c|c|}
\hline \multirow{2}{*}{ ID* } & \multicolumn{5}{|c|}{ Height measurement (in.) } & \multirow{2}{*}{$\begin{array}{c}\text { Average } \\
\text { diameter } \\
\text { (in.) }\end{array}$} & \multirow{2}{*}{$\begin{array}{c}\text { Allowable } \\
\text { deviation } \\
\text { (in.) }\end{array}$} & \multirow{2}{*}{$\begin{array}{c}\text { Max } \\
\text { absolute } \\
\text { deviation } \\
\text { (in.) }\end{array}$} & \multirow{2}{*}{$\begin{array}{c}\begin{array}{c}\text { Deviation } \\
\text { from }\end{array} \\
\text { parallelness } \\
\text { (degrees)** }\end{array}$} \\
\hline & 1 & 2 & 3 & 4 & Center & & & & \\
\hline $2 \mathrm{M}$ & 0.250 & 0.245 & 0.242 & 0.231 & 0.248 & 3.0240 & 0.013 & 0.017 & 0.64 \\
\hline $2 \mathrm{~B}$ & 0.221 & 0.220 & 0.220 & 0.221 & 0.220 & 3.0165 & 0.013 & 0.001 & 0.04 \\
\hline $3 \mathrm{~T}$ & 0.270 & 0.250 & 0.240 & 0.250 & 0.260 & 3.0280 & 0.013 & 0.020 & 0.76 \\
\hline $3 \mathrm{M}$ & 0.300 & 0.300 & 0.290 & 0.290 & 0.300 & 3.0172 & 0.013 & 0.010 & 0.38 \\
\hline $4 \mathrm{~T}$ & 0.241 & 0.261 & 0.241 & 0.240 & 0.241 & 3.0158 & 0.013 & 0.020 & 0.76 \\
\hline $4 \mathrm{~B}$ & 0.150 & 0.160 & 0.151 & 0.162 & 0.150 & 3.0160 & 0.013 & 0.012 & 0.46 \\
\hline $5 \mathrm{M}$ & 0.105 & 0.110 & 0.055 & 0.083 & 0.095 & 3.0257 & 0.013 & 0.040 & 1.51 \\
\hline $5 \mathrm{~B}$ & 0.371 & 0.361 & 0.381 & 0.361 & 0.371 & 3.0207 & 0.013 & 0.010 & 0.38 \\
\hline $6 \mathrm{~B}$ & 0.990 & 0.980 & 0.990 & 0.980 & 0.990 & 3.0280 & 0.013 & 0.010 & 0.38 \\
\hline $7 \mathrm{~T}$ & 0.370 & 0.362 & 0.369 & 0.370 & 0.363 & 3.0227 & 0.013 & 0.007 & 0.27 \\
\hline $9 \mathrm{~T}$ & 0.110 & 0.112 & 0.100 & 0.118 & 0.112 & 3.0237 & 0.013 & 0.012 & 0.45 \\
\hline $9 \mathrm{M}$ & 0.889 & 0.882 & 0.892 & 0.929 & 0.935 & 3.0192 & 0.013 & 0.053 & 2.01 \\
\hline $10 \mathrm{~T}$ & 0.190 & 0.190 & 0.180 & 0.170 & 0.190 & 3.0223 & 0.013 & 0.020 & 0.76 \\
\hline $10 \mathrm{~B}$ & 0.355 & 0.362 & 0.378 & 0.373 & 0.359 & 3.0245 & 0.013 & 0.019 & 0.72 \\
\hline $11 \mathrm{~T}$ & 0.689 & 0.690 & 0.716 & 0.720 & 0.700 & 3.0278 & 0.013 & 0.020 & 0.76 \\
\hline $11 \mathrm{~B}$ & 0.342 & 0.337 & 0.333 & 0.332 & 0.330 & 3.0163 & 0.013 & 0.012 & 0.46 \\
\hline $12 \mathrm{M}$ & 0.141 & 0.130 & 0.160 & 0.171 & 0.150 & 3.0282 & 0.013 & 0.021 & 0.79 \\
\hline $13 \mathrm{~T}$ & 0.205 & 0.192 & 0.200 & 0.217 & 0.191 & 3.0278 & 0.013 & 0.026 & 0.98 \\
\hline
\end{tabular}

*For the sake of brevity, only the specimen position is given

**Bolded measurements indicate specimens not meeting the recommended 0.5 degree parallelness tolerance. 


\section{APPENDIX B X-Ray CT Scan Settings}

Table B.1. Settings for X-ray CT scans performed in December 2015.

\begin{tabular}{lccccccccc}
\hline \hline ID* & $\begin{array}{c}\text { Voltage } \\
(\mathbf{k V})\end{array}$ & $\begin{array}{c}\text { Current } \\
(\boldsymbol{\mu} \mathbf{A})\end{array}$ & $\begin{array}{c}\text { Power } \\
(\mathbf{W})\end{array}$ & $\begin{array}{c}\text { Isowatt } \\
\text { Setting }\end{array}$ & $\begin{array}{c}\boldsymbol{D}_{\mathbf{1}} \\
(\mathbf{i n .})\end{array}$ & $\begin{array}{c}\boldsymbol{D}_{\mathbf{2}} \\
(\mathbf{i n .})\end{array}$ & $\begin{array}{c}\text { No. of } \\
\text { view } \\
\text { steps }\end{array}$ & $\begin{array}{c}\text { No. of } \\
\text { averaged } \\
\text { frames }\end{array}$ & $\begin{array}{c}\text { Variation } \\
\text { limit (\%) }\end{array}$ \\
\hline 2M & 185 & 270 & 49.95 & Off & $19-1 / 2$ & $37-3 / 8$ & 720 & 1 & 2 \\
2B & 185 & 270 & 49.95 & Off & $19-1 / 2$ & $37-3 / 8$ & 720 & 1 & 2 \\
3M & 185 & 270 & 49.95 & Off & $19-1 / 2$ & $37-3 / 8$ & 720 & 1 & 2 \\
3T & 185 & 270 & 49.95 & Off & $19-1 / 2$ & $37-3 / 8$ & 720 & 1 & 2 \\
2M-Cal & 120 & 683.3 & 82 & On & $19-1 / 2$ & $37-3 / 8$ & 60 & 1 & 2 \\
5T & 185 & 290 & 53.65 & Off & $19-1 / 2$ & $37-3 / 8$ & 720 & 1 & 2 \\
3B & 185 & 290 & 53.65 & Off & $19-1 / 2$ & $37-3 / 8$ & 720 & 1 & 2 \\
7B & 185 & 290 & 53.65 & Off & $19-1 / 2$ & $37-3 / 8$ & 720 & 1 & 2 \\
6M & 185 & 290 & 53.65 & Off & $19-1 / 2$ & $37-3 / 8$ & 720 & 1 & 2 \\
5T-Cal & 150 & 270 & 40.50 & Off & $19-1 / 2$ & $37-3 / 8$ & 60 & 1 & 2 \\
\hline \hline
\end{tabular}

*For the sake of brevity, only the specimen position is given. Entries suffixed with "-Cal" are calibration scans (see Section 3.2.4). 
Table B.2. Settings for X-ray CT scans performed in February 2016.

\begin{tabular}{|c|c|c|c|c|c|c|c|c|c|}
\hline ID* & $\begin{array}{c}\text { Voltage } \\
(\mathbf{k V})\end{array}$ & $\begin{array}{c}\text { Current } \\
(\boldsymbol{\mu A})\end{array}$ & $\begin{array}{l}\text { Power } \\
\text { (W) }\end{array}$ & $\begin{array}{l}\text { Isowatt } \\
\text { Setting }\end{array}$ & $\begin{array}{c}D_{1} \\
\text { (in.) }\end{array}$ & $\begin{array}{c}D_{2} \\
\text { (in.) }\end{array}$ & 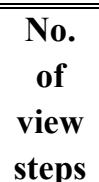 & $\begin{array}{c}\text { No. of } \\
\text { averaged } \\
\text { frames }\end{array}$ & $\begin{array}{l}\text { Variation } \\
\text { limit (\%) }\end{array}$ \\
\hline $4 \mathrm{~B}$ & 185 & 308.1 & 57 & On & $\mathrm{N} / \mathrm{R}^{* *}$ & $\mathrm{~N} / \mathrm{R}$ & 720 & 1 & 2 \\
\hline $4 \mathrm{~T}$ & 185 & 308.1 & 57 & On & $\mathrm{N} / \mathrm{R}$ & $\mathrm{N} / \mathrm{R}$ & 720 & 1 & 2 \\
\hline $6 \mathrm{~B}$ & 185 & 308.1 & 57 & On & $\mathrm{N} / \mathrm{R}$ & $\mathrm{N} / \mathrm{R}$ & 720 & 1 & 2 \\
\hline $5 \mathrm{M}$ & 185 & 308.1 & 57 & On & $\mathrm{N} / \mathrm{R}$ & $\mathrm{N} / \mathrm{R}$ & 720 & 1 & 2 \\
\hline $5 \mathrm{~B}$ & 185 & 308.1 & 57 & On & $\mathrm{N} / \mathrm{R}$ & $\mathrm{N} / \mathrm{R}$ & 720 & 1 & 2 \\
\hline 9T & 185 & 308.1 & 57 & On & $\mathrm{N} / \mathrm{R}$ & $\mathrm{N} / \mathrm{R}$ & 720 & 1 & 2 \\
\hline $7 \mathrm{~T}$ & 185 & 308.1 & 57 & On & $\mathrm{N} / \mathrm{R}$ & $\mathrm{N} / \mathrm{R}$ & 720 & 1 & 2 \\
\hline 6B-Cal & 120 & 683.3 & 82 & On & $\mathrm{N} / \mathrm{R}$ & $\mathrm{N} / \mathrm{R}$ & 60 & 1 & 2 \\
\hline $9 \mathrm{M}$ & 185 & 308.1 & 57 & On & $19-1 / 2$ & $37-3 / 8$ & 720 & 1 & 2 \\
\hline $13 \mathrm{~T}$ & 185 & 308.1 & 57 & On & $19-1 / 2$ & $37-3 / 8$ & 720 & 1 & 2 \\
\hline 13T-Cal & 120 & 683.3 & 82 & On & $19-1 / 2$ & $37-3 / 8$ & 60 & 1 & 2 \\
\hline $11 \mathrm{~T}$ & 162 & 493.8 & 80 & On & $19-1 / 2$ & $37-3 / 8$ & 720 & 1 & 2 \\
\hline $10 \mathrm{~B}$ & 162 & 493.8 & 80 & On & $19-1 / 2$ & $37-3 / 8$ & 720 & 1 & 2 \\
\hline $10 \mathrm{~T}$ & 162 & 493.8 & 80 & On & $19-1 / 2$ & $37-3 / 8$ & 720 & 1 & 2 \\
\hline $12 \mathrm{M}$ & 162 & 493.8 & 80 & On & $19-1 / 2$ & $37-3 / 8$ & 720 & 1 & 2 \\
\hline $11 \mathrm{~B}$ & 162 & 493.8 & 80 & On & $19-1 / 2$ & $37-3 / 8$ & 720 & 1 & 2 \\
\hline $11 \mathrm{M}$ & 162 & 493.8 & 80 & On & $26-5 / 8$ & 30 & 720 & 1 & 2 \\
\hline $8 \mathrm{~B}$ & 162 & 493.8 & 80 & On & $26-5 / 8$ & 30 & 720 & 1 & 2 \\
\hline $14 \mathrm{~T}$ & 162 & 493.8 & 80 & On & $26-5 / 8$ & 30 & 720 & 1 & 2 \\
\hline $14 \mathrm{M}$ & 162 & 493.8 & 80 & On & $26-5 / 8$ & 30 & 720 & 1 & 2 \\
\hline $12 \mathrm{~B}$ & 162 & 493.8 & 80 & On & $26-5 / 8$ & 30 & 720 & 1 & 2 \\
\hline $8 \mathrm{~T}$ & 162 & 493.8 & 80 & On & $26-5 / 8$ & 30 & 720 & 1 & 2 \\
\hline $13 \mathrm{~B}$ & 162 & 493.8 & 80 & On & $26-5 / 8$ & 30 & 720 & 1 & 2 \\
\hline $13 \mathrm{M}$ & 162 & 493.8 & 80 & On & $26-5 / 8$ & 30 & 720 & 1 & 2 \\
\hline $8 \mathrm{M}$ & 162 & 493.8 & 80 & On & $26-5 / 8$ & 30 & 720 & 1 & 2 \\
\hline $15 \mathrm{~T}$ & 162 & 493.8 & 80 & On & $26-5 / 8$ & 30 & 720 & 1 & 2 \\
\hline $10 \mathrm{M}$ & 162 & 493.8 & 80 & On & $26-5 / 8$ & 30 & 720 & 1 & 2 \\
\hline STAT-Cal & 125 & 656.0 & 82 & On & $26-5 / 8$ & 30 & 60 & 1 & 2 \\
\hline
\end{tabular}

*For the sake of brevity, only the specimen position is given. Entries suffixed with “-Cal" are calibration scans (see Section 3.2.4).

**N/R indicates the measurement was not recorded. 
Table B.3. CT scan volume reconstruction settings.

\begin{tabular}{|c|c|c|}
\hline ID* & Voxel field size & $\begin{array}{c}\text { Resolution } \\
\text { ( } \mu \mathrm{m})\end{array}$ \\
\hline $2 \mathrm{M}$ & $1949 \times 1996 \times 1949$ & 42.08 \\
\hline $2 \mathrm{~B}$ & $1949 \times 1996 \times 1949$ & 42.08 \\
\hline $3 \mathrm{M}$ & $1949 \times 1996 \times 1949$ & 42.08 \\
\hline $3 \mathrm{~T}$ & $1949 \times 1996 \times 1949$ & 42.08 \\
\hline $5 \mathrm{~T}$ & $1603 \times 3069 \times 1603$ & 51.15 \\
\hline $3 B$ & $1603 \times 2952 \times 1603$ & 51.15 \\
\hline $7 \mathrm{~B}$ & $1603 \times 3011 \times 1603$ & 51.15 \\
\hline $6 \mathrm{M}$ & $1603 \times 3011 \times 1603$ & 51.15 \\
\hline $4 \mathrm{~B}$ & $2099 \times 2201 \times 2099$ & 39.07 \\
\hline $4 \mathrm{~T}$ & $2099 \times 2201 \times 2099$ & 39.07 \\
\hline $6 \mathrm{~B}$ & $2099 \times 2201 \times 2099$ & 39.07 \\
\hline $5 \mathrm{M}$ & $2098 \times 2149 \times 2098$ & 39.08 \\
\hline $5 B$ & $2098 \times 2149 \times 2098$ & 39.08 \\
\hline $9 \mathrm{~T}$ & $2098 \times 2149 \times 2098$ & 39.08 \\
\hline $7 \mathrm{~T}$ & $2098 \times 2149 \times 2098$ & 39.08 \\
\hline $9 \mathrm{M}$ & $1950 \times 1997 \times 1950$ & 42.06 \\
\hline $13 \mathrm{~T}$ & $1950 \times 1997 \times 1950$ & 42.06 \\
\hline $11 \mathrm{~T}$ & $1950 \times 1997 \times 1950$ & 42.06 \\
\hline $10 \mathrm{~B}$ & $1950 \times 1997 \times 1950$ & 42.06 \\
\hline $10 \mathrm{~T}$ & $1950 \times 1997 \times 1950$ & 42.06 \\
\hline $12 \mathrm{M}$ & $1950 \times 1997 \times 1950$ & 42.06 \\
\hline $11 \mathrm{~B}$ & $1950 \times 1997 \times 1950$ & 42.06 \\
\hline $11 \mathrm{M}$ & $1436 \times 2750 \times 1436$ & 57.09 \\
\hline $8 B$ & $1436 \times 2750 \times 1436$ & 57.09 \\
\hline $14 \mathrm{~T}$ & $1436 \times 2750 \times 1436$ & 57.09 \\
\hline $14 \mathrm{M}$ & $1436 \times 2750 \times 1436$ & 57.09 \\
\hline $12 \mathrm{~B}$ & $1436 \times 2750 \times 1436$ & 57.09 \\
\hline $8 \mathrm{~T}$ & $1436 \times 2750 \times 1436$ & 57.09 \\
\hline $13 \mathrm{~B}$ & $1436 \times 2750 \times 1436$ & 57.09 \\
\hline $13 \mathrm{M}$ & $1436 \times 2750 \times 1436$ & 57.09 \\
\hline $8 \mathrm{M}$ & $1436 \times 2750 \times 1436$ & 57.09 \\
\hline $15 \mathrm{~T}$ & $1436 \times 2750 \times 1436$ & 57.09 \\
\hline $10 \mathrm{M}$ & $1436 \times 2750 \times 1436$ & 57.09 \\
\hline
\end{tabular}

*For the sake of brevity, only the specimen position is given. 


\section{APPENDIX C Code Scaling Pilot Study}

The LSFfiberOrient function (Flanders 2014; modifications by Oesch 2014f) was run on the Portage cluster using 1, 2, 4, 8, and 11 cores to test its scaling properties. Data from $\mathrm{X}-\mathrm{D}-2 \mathrm{M}$ was used for all test runs. Timing results from these runs are shown in Figure C.1. As can be seen, the elapsed time (total time required to complete) decreases as more cores are used. However, the overhead required to manage the computation also increases, as indicated by the CPU time series in the figure. The CPU time is a measure of the total use of resources: if four processors execute a task in 15 minutes, the elapsed time is 15 minutes, but the CPU time is 1 hour. The figure indicates that the most efficient use of resources occurs when using two processors, as the CPU time is minimized at that point.

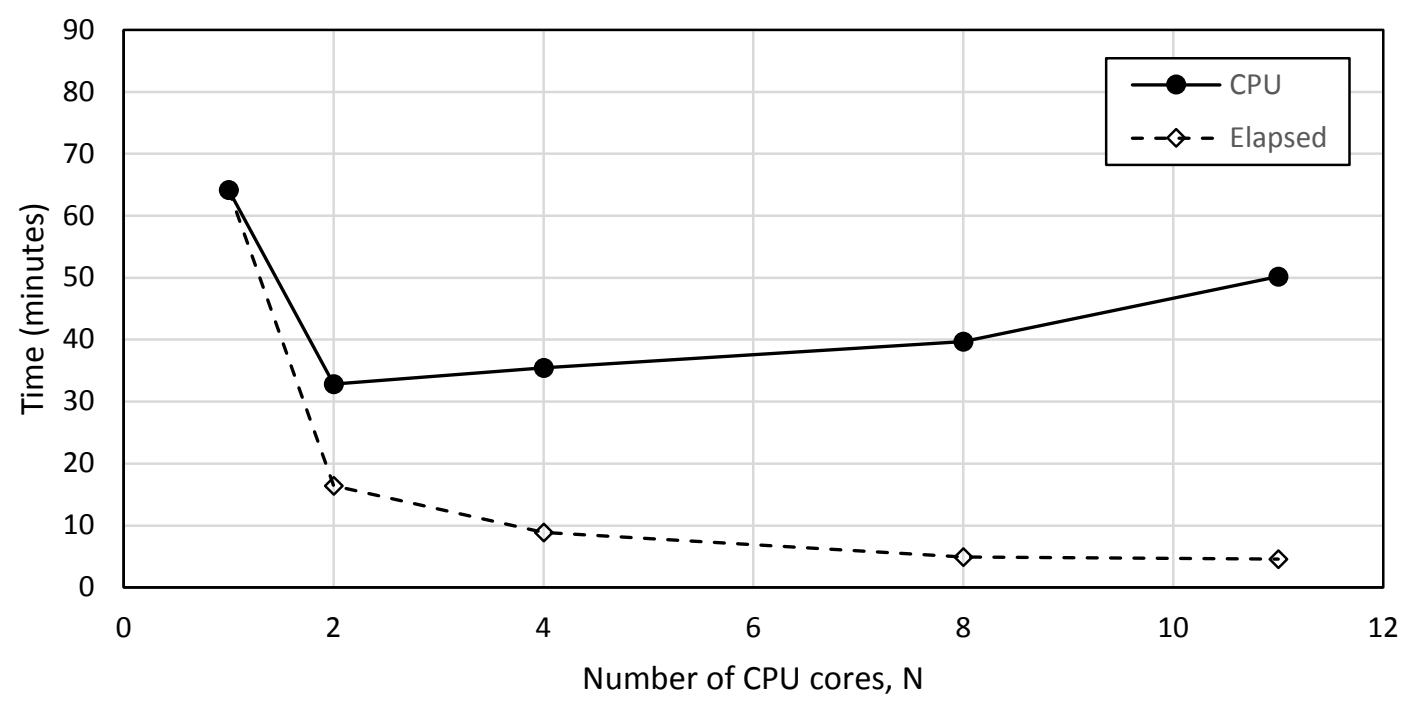

Figure C.1. Timing measurements for LSFfiberOrient function for multiple cores. Elapsed time is the total time taken to run; CPU time is the number of cores times the elapsed time. 


\section{APPENDIX D Specimen Dimensions}

Table D.1. Quasistatic compression test specimen dimensions.

\begin{tabular}{cccccccccc}
\hline \hline \multirow{2}{*}{ Specimen ID* } & \multicolumn{4}{c}{ Length (in.) } & \multicolumn{5}{c}{ Diameter (in.) } \\
& $\mathbf{1}$ & $\mathbf{2}$ & $\mathbf{3}$ & Avg. & $\mathbf{1}$ & $\mathbf{2}$ & $\mathbf{3}$ & Avg. & L/D \\
\hline 3B & 5.6270 & 5.6280 & 5.6280 & 5.628 & 3.0130 & 3.0120 & 3.0115 & 3.012 & 1.9 \\
5T & 5.8775 & 5.8760 & 5.8785 & 5.877 & 3.0180 & 3.0175 & 3.0150 & 3.017 & 1.9 \\
6M & 5.7250 & 5.7325 & 5.7300 & 5.729 & 3.0230 & 3.0220 & 3.0230 & 3.023 & 1.9 \\
7M & 6.0965 & 6.0925 & 6.0990 & 6.096 & 3.0210 & 3.0220 & 3.0265 & 3.023 & 2.0 \\
7B & 5.7965 & 5.7940 & 5.7905 & 5.794 & 3.0075 & 3.0130 & 3.0120 & 3.011 & 1.9 \\
8T & 5.5970 & 5.5975 & 5.5990 & 5.598 & 3.0190 & 3.0205 & 3.0195 & 3.020 & 1.9 \\
8M & 5.9565 & 5.9545 & 5.9585 & 5.957 & 3.0095 & 3.0115 & 3.0105 & 3.011 & 2.0 \\
8B & 5.9945 & 5.9935 & 5.9875 & 5.992 & 3.0195 & 3.0205 & 3.0220 & 3.021 & 2.0 \\
9B & 5.6790 & 5.6810 & 5.6785 & 5.680 & 3.0270 & 3.0255 & 3.0205 & 3.024 & 1.9 \\
10M & 5.6775 & 5.6715 & 5.6765 & 5.675 & 3.0250 & 3.0220 & 3.0215 & 3.023 & 1.9 \\
$11 \mathrm{M}$ & 6.1150 & 6.1165 & 6.1165 & 6.116 & 3.0105 & 3.0950 & 3.0120 & 3.039 & 2.0 \\
12T & 5.7815 & 5.7815 & 5.7820 & 5.782 & 3.0130 & 3.0140 & 3.0135 & 3.014 & 1.9 \\
12B & 5.6925 & 5.6890 & 5.6935 & 5.692 & 3.0205 & 3.0230 & 3.0235 & 3.022 & 1.9 \\
13M & 5.7565 & 5.7440 & 5.7460 & 5.749 & 3.0240 & 3.0205 & 3.0205 & 3.022 & 1.9 \\
13B & 5.7525 & 5.7565 & 5.7540 & 5.754 & 3.0165 & 3.0155 & 3.0185 & 3.017 & 1.9 \\
14T & 5.4940 & 5.4915 & 5.4960 & 5.494 & 3.0115 & 3.0210 & 3.0170 & 3.017 & 1.8 \\
$14 \mathrm{M}$ & 5.9270 & 5.9280 & 5.9240 & 5.926 & 3.0175 & 3.0155 & 3.0165 & 3.017 & 2.0 \\
$15 \mathrm{~T}$ & 5.6305 & 5.6335 & 5.6395 & 5.635 & 3.0170 & 3.0190 & 3.0230 & 3.020 & 1.9 \\
\hline \hline
\end{tabular}

*For the sake of brevity, only the specimen position is given.

Table D.2. Additional quasistatic compression test specimen dimensions.

\begin{tabular}{cccccccccc}
\hline \hline \multirow{2}{*}{ Specimen ID** } & \multicolumn{4}{c}{ Length (in.) } & \multicolumn{4}{c}{ Diameter (in.) } & \multirow{2}{*}{ L/D } \\
& $\mathbf{1}$ & $\mathbf{2}$ & $\mathbf{3}$ & Avg. & $\mathbf{1}$ & $\mathbf{2}$ & $\mathbf{3}$ & Avg. & \\
\hline 1T & 5.6120 & 5.6030 & 5.6005 & 5.605 & 3.0220 & 3.0215 & 3.0240 & 3.023 & 1.9 \\
1B & 5.7015 & 5.6980 & 5.7075 & 5.702 & 3.0120 & 3.0095 & 3.0060 & 3.009 & 1.9 \\
2T & 6.0585 & 6.0550 & 6.0565 & 6.057 & 3.0055 & 3.0135 & 3.0120 & 3.010 & 2.0 \\
15B & 6.1190 & 6.1215 & 6.1265 & 6.122 & 3.0180 & 3.0310 & 3.0205 & 3.023 & 2.0 \\
16T & 5.7250 & 5.7260 & 5.7275 & 5.726 & 3.0200 & 3.0240 & 3.0225 & 3.022 & 1.9 \\
16M & 5.9165 & 5.8995 & 5.8930 & 5.903 & 3.0165 & 3.0200 & 3.0230 & 3.020 & 2.0 \\
\hline \hline
\end{tabular}

*For the sake of brevity, only the specimen position is given. 
Table D.3. Dynamic compression test specimen dimensions.

\begin{tabular}{cccccccccc}
\hline \multirow{2}{*}{ Specimen ID* } & \multicolumn{4}{c}{ Length (in.) } & \multicolumn{7}{c}{ Diameter (in.) } \\
& $\mathbf{1}$ & $\mathbf{2}$ & $\mathbf{3}$ & Avg. & $\mathbf{1}$ & $\mathbf{2}$ & $\mathbf{3}$ & Avg. & L/D \\
\hline 2M & 3.0305 & 3.0315 & 3.0305 & 3.031 & 3.0250 & 3.0220 & 3.0250 & 3.024 & 1.0 \\
2B & 3.0290 & 3.0280 & 3.0340 & 3.030 & 3.0175 & 3.0100 & 3.0220 & 3.017 & 1.0 \\
3T & 3.0325 & 3.0315 & 3.0345 & 3.033 & 3.0335 & 3.0300 & 3.0205 & 3.028 & 1.0 \\
3M & 3.0390 & 3.0345 & 3.0360 & 3.037 & 3.0155 & 3.0195 & 3.0165 & 3.017 & 1.0 \\
4T & 3.0370 & 3.0280 & 3.0295 & 3.032 & 3.0255 & 3.0070 & 3.0150 & 3.016 & 1.0 \\
4B & 3.0185 & 3.0200 & 3.0215 & 3.020 & 3.0140 & 3.0165 & 3.0175 & 3.016 & 1.0 \\
5M & 3.0155 & 3.0095 & 3.0105 & 3.012 & 3.0290 & 3.0235 & 3.0245 & 3.026 & 1.0 \\
5B & 3.0400 & 3.0380 & 3.0445 & 3.041 & 3.0230 & 3.0185 & 3.0205 & 3.021 & 1.0 \\
6B & 3.0035 & 3.0050 & 3.0065 & 3.005 & 3.0310 & 3.0260 & 3.0270 & 3.028 & 1.0 \\
7T & 3.0430 & 3.0460 & 3.0425 & 3.044 & 3.0205 & 3.0210 & 3.0265 & 3.023 & 1.0 \\
9T & 3.0175 & 3.0180 & 3.0195 & 3.018 & 3.0280 & 3.0220 & 3.0210 & 3.024 & 1.0 \\
9M & 2.9950 & 2.9945 & 2.9975 & 2.996 & 3.0185 & 3.0175 & 3.0215 & 3.019 & 1.0 \\
10T & 3.0280 & 3.0265 & 3.0245 & 3.026 & 3.0215 & 3.0230 & 3.0225 & 3.022 & 1.0 \\
10B & 3.0420 & 3.0450 & 3.0415 & 3.043 & 3.0280 & 3.0260 & 3.0195 & 3.025 & 1.0 \\
11T & 2.9975 & 2.9850 & 2.9875 & 2.990 & 3.0455 & 3.0180 & 3.0200 & 3.028 & 1.0 \\
11B & 3.0430 & 3.0400 & 3.0390 & 3.041 & 3.0155 & 3.0195 & 3.0140 & 3.016 & 1.0 \\
$12 \mathrm{M}$ & 3.0245 & 3.0175 & 3.0205 & 3.021 & 3.0230 & 3.0285 & 3.0330 & 3.028 & 1.0 \\
$13 \mathrm{~T}$ & 3.0240 & 3.0235 & 3.0245 & 3.024 & 3.0290 & 3.0230 & 3.0315 & 3.028 & 1.0 \\
\hline \hline
\end{tabular}

*For the sake of brevity, only the specimen position is given.

Table D.4. Dynamic compression test specimen weights.

\begin{tabular}{cccccc}
\hline $\begin{array}{c}\text { Specimen } \\
\text { ID* }\end{array}$ & $\begin{array}{c}\text { Weight } \\
\text { (lbf) }\end{array}$ & $\begin{array}{c}\text { Specific } \\
\text { weight (pcf) }\end{array}$ & $\begin{array}{c}\text { Specimen } \\
\text { ID }\end{array}$ & $\begin{array}{c}\text { Weight } \\
\text { (lbf) }\end{array}$ & $\begin{array}{c}\text { Specific } \\
\text { weight (pcf) }\end{array}$ \\
\hline 2M & 2.01 & 160 & $7 \mathrm{~T}$ & 2.00 & 158 \\
2B & 1.98 & 158 & $9 \mathrm{~T}$ & 2.00 & 159 \\
3T & 2.03 & 161 & $9 \mathrm{M}$ & 1.96 & 158 \\
3M & 1.98 & 158 & $10 \mathrm{~T}$ & 2.00 & 159 \\
4T & 2.00 & 160 & $10 \mathrm{~B}$ & 2.00 & 158 \\
4B & 1.99 & 159 & $11 \mathrm{~T}$ & 1.96 & 157 \\
5M & 1.97 & 157 & $11 \mathrm{~B}$ & 2.00 & 159 \\
5B & 2.00 & 159 & $12 \mathrm{M}^{* *}$ & - & - \\
6B & 1.98 & 158 & $13 \mathrm{~T}$ & 2.00 & 159 \\
\hline \hline
\end{tabular}

*For the sake of brevity, only the specimen position is given.

**No weight recorded. The average specific weight was used in analysis. 


\section{APPENDIX E Numerical Differentiation}

This appendix contains code used in determining strain acceleration. First derivatives of strain rate were computed numerically using a Savitzky-Golay filter for smoothing, which was implemented by the MATLAB function reproduced below.

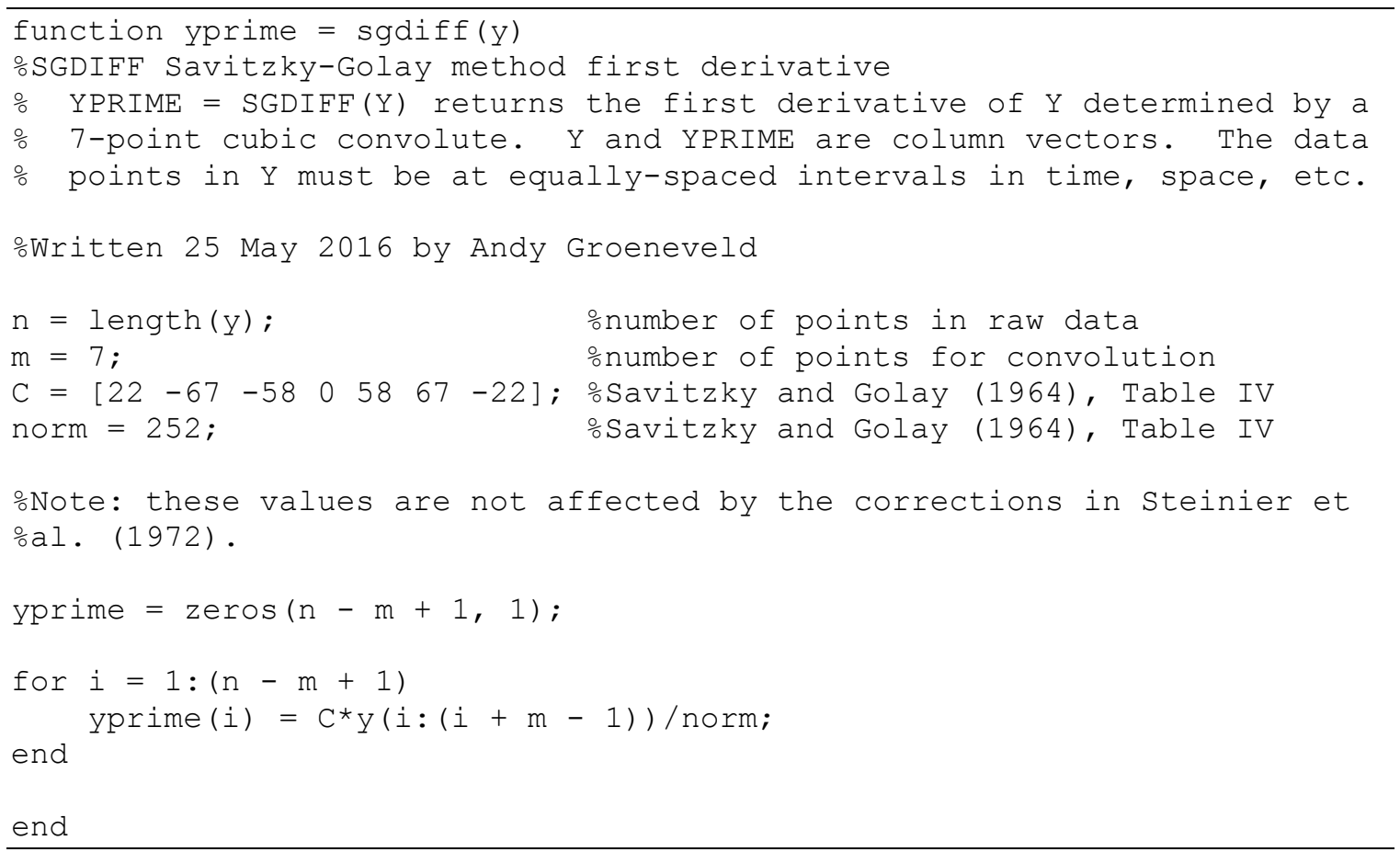




\section{APPENDIX F Permission for Use Of Copyrighted Material}

The email below pertains to Figure 4.2.

\section{Permission to use $C T$ reconstruction image in thesis}

Burchfield, Charles A ERDC-GSL-MS <Charles.A.Burchfield@usace.army.mil>

To: Andrew Groeneveld <abgroene@mtu.edu>

Fri, Jun 10, 2016 at 9:16 AM

Hey Andy,

Absolutely you have my permission! The work was done for you, and we were trying to get you some good images to use.

Congratulations on finishing up! Look forward to having you in Vicksburg. Also, looking forward to reading your thesis.

Tell Dr. Ahlborn that Amanda and I said hello!

Have a good one,

Charlie

Sent from my BlackBerry 10 smartphone.

From: Andrew Groeneveld

Sent: Friday, June 10, 2016 7:32 AM

To: Burchfield, Charles A ERDC-GSL-MS

Subject: [EXTERNAL] Permission to use CT reconstruction image in thesis

Hi Charlie,

I hope you are doing well. Thank you again for your help with CT scanning

I am asking permission to use the screenshot of $2 \mathrm{M}$ DYN's reconstruction, which you sent on the SD card in my thesis. The image is also attached for reference. Michigan Tech requires that I document permission to use any material that I did not produce myself. An email is sufficient for their purposes.

Barring any distribution limitations, the completed thesis will be available online through Michigan Tech and through ProQuest.

Thank you very much! Have a good weekend.

Andy

Andrew Groeneveld

Civil Engineering MS Student

Michigan Technological University

Cell: +1 2404499364 